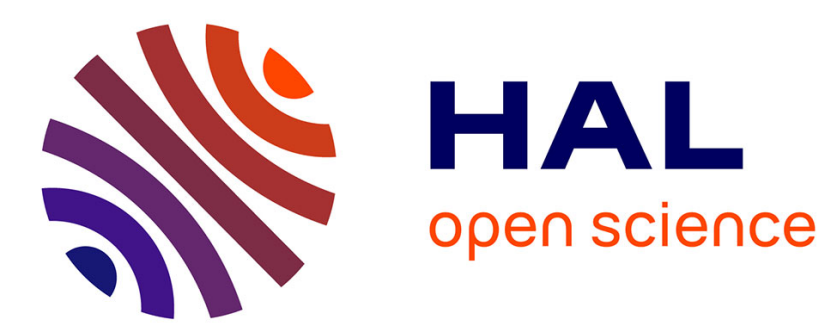

\title{
Arclength continuation methods and applications to 2D drift-diffusion semiconductor equations
}

Abderrazzak El Boukili, Americo Marrocco

\section{To cite this version:}

Abderrazzak El Boukili, Americo Marrocco. Arclength continuation methods and applications to 2D drift-diffusion semiconductor equations. [Research Report] RR-2546, INRIA. 1995. inria-00074132

\section{HAL Id: inria-00074132 \\ https://hal.inria.fr/inria-00074132}

Submitted on 24 May 2006

HAL is a multi-disciplinary open access archive for the deposit and dissemination of scientific research documents, whether they are published or not. The documents may come from teaching and research institutions in France or abroad, or from public or private research centers.
L'archive ouverte pluridisciplinaire HAL, est destinée au dépôt et à la diffusion de documents scientifiques de niveau recherche, publiés ou non, émanant des établissements d'enseignement et de recherche français ou étrangers, des laboratoires publics ou privés. 


\section{Arclength continuation methods and applications to $2 D$ drift-diffusion semiconductor equations}

Abderrazzak EL BOUKILI, Americo MARROCCO

$\mathbf{N}^{\circ} 2546$

Mai 1995

PROGRAMME 6 



\title{
RIN RIA
}

\section{Arclength continuation methods and applications to 2D drift-diffusion semiconductor equations}

\author{
Abderrazzak EL BOUKILI*, Americo MARROCCO ** \\ Programme 6 - Calcul scientifique, modélisation et logiciel numérique \\ Projet Menusin
}

Rapport de recherche $\mathrm{n}^{\circ} 2546$ - Mai 1995 - 100 pages

\begin{abstract}
In this paper, the homotopy deformation method to solve the nonlinear stationary semiconductor equations with Fermi-Dirac statistic is used. This method introduces an artificial transient problem. The time discretization is based on the nonlinear implicit scheme with local time steps. In order to have an automatic adaptation of local time step parameters, we introduce arclength predictor-corrector continuation methods. The fondamental goal of these methods is to overcome the unstabilities or the failure of the classical Newton-Raphson's schemes which appear when the nonlinearity is Strong or near Limit or Bifurcation points. The approximate procedure of our system using a Galerkin method that makes use of a mixed finite element approach is used. A peculiar feature of this mixed formulation is that the electric displacement $D$ and the current densities $j_{n}$ and $j_{p}$ for electrons and holes, are taken as unknowns, together with the potential $\phi$ and quasi_Fermi levels $\phi_{n}$ and $\phi_{p}$. This allows $D, j_{n}$ and $j_{p}$ to be determined directly and accurately. The above algorithms appear to be efficient, robust and to give satisfactory results. Numerical results are presented, in one and two dimension, for some realistic devices : an Heterojunction Diode (quasi-1D problem) and an Heterojunction Bipolar Transistor (HBT) working in amplifier mode.
\end{abstract}

Key-words: Arclength predictor-corrector continuation, automatic adaptation, nonlinear implicit schemes, local time steps, mixed finite elements, heterojunction semiconductors, nonlinear Poisson's equation, transport equations ( for electrons and holes) with variable coefficients, drift-diffusion.

(Résumé : tsvp)

${ }^{*}$ Email : Abderrazzak.ElBoukili@inria.fr

** Email : Americo.Marrocco@inria.fr 


\section{Méthodes de continuation de Pseudo Longueur d'Arc et applications aux équations de dérive-diffusion pour les semi-conducteurs.}

Résumé : Dans cet article, on utilise la méthode de déformation par homotopie pour résoudre le problème stationnaire des équations non linéaires gouvernant le fonctionnement électrique des dispositifs semi-conducteurs à hétérojonction en statistique de Fermi-Dirac. La méthode introduit un problème transitoire artificiel. La discrétisation en temps est basée sur un schéma implicite non linéaire avec pas de temps locaux. De façon à pouvoir faire une adaptation automatique des pas de temps, on introduit les méthodes de continuation de type "arclength". Le but essentiel de ces méthodes est de surmonter les instabilités ou les échecs des méthodes classiques de type Newton-Raphson qui peuvent apparaître lorsque la non linéarité est forte ou lorsque il existe des points de retournements (points limites ou points de bifurcation). L'approximation en espace du système d'équations est faite via une méthode de Galerkin de type éléments finis mixtes. Un point important de cette formulation mixte est que le vecteur déplacement électrique $D$, ainsi que les vecteurs densités de courant $j_{n}$ et $j_{p}$ sont considérés comme des inconnues au même titre que le potentiel électrostatique $\phi$ et les quasi-niveaux de Fermi $\phi_{n}$ et $\phi_{p}$. Cette approche permet de déterminer $D, j_{n}, j_{p}$ directement et avec bonne précision. Les algorithmes de résolution mentionnés ci-dessus sont robustes, souples et efficaces. On présente des résultats numériques concernant certains dispositifs réalistes : une Diode à hétérojonction (problème quasi-1D) et un Transistor bipolaire à hétérojonctions (TBH) fonctionnant en mode amplificateur (dispositif bidimensionnel).

Mots-clé : Méthodes de continuation par prédiction-correction de type "arclength", adaptation automatique, schémas implicites non linéaires, pas de temps locaux, éléments finis mixtes, semi-conducteurs à hétérojonctions, équation de Poisson non linéaire, équations de transport (des électrons et trous) à coefficients variables, dérive-diffusion. 


\section{Introduction}

We show in this paper how a strongly nonlinear semiconductor equations can be solved via homotopy deformation methods combined with the arclength predictorcorrector continuation procedures. Using the deformation method, we write the system in an artificial transient form. The time step in the nonlinear implicit discretization is treated as a parameter. An automatic and rigorous adaptation of this latter to the nonlinearity of the problem is assured by the arclength continuation methods. Continuation, embedding or homotopy methods have long served as useful tools in modern mathematics. Their use can be traced back at least to Poincaré (1881-1886) [1], Klein (1882-1883) [2] and Bernstein (1910) [3]. Leray and Schauder (1934) [4] refined the tool and presented it as a global result in topology. The use of homotopy deformation to solve nonlinear systems of equations may be traced back at least to Lahaye (1934) [5]. The classical embedding methods may be regarded as a forerunner of the predictor-corrector methods. The homotopy method consists of the following. Suppose one wishes to obtain a solution to a system of $\mathrm{N}$ nonlinear equations in $\mathrm{N}$ variables, say

$$
F(u)=0
$$

where $F: \mathbb{R}^{N} \rightarrow \mathbb{R}^{N}$ is a map which is assumed to be smooth. Let us consider the situation in which we havn't a sufficient a priori knowledge about zero point of $F$. Certainly, if on the contrary a good approximation $u_{1}$ of a zero point $\bar{u}$ of $\mathrm{F}$ is available, it is advisable to calculate $\bar{u}$ using a standard Newton-Raphson's type algorithm defined by an iteration formula such as

$$
u_{n+1}=u_{n}-A_{n}^{-1} F\left(u_{n}\right) ; n=1,2, \ldots
$$

where $A_{n}$ is some reasonable approximation of the Jacobian $F^{\prime}\left(u_{n}\right)$.

Since in most applications such a priori knowledge is not available, the iteration (2) will often fail, because poor starting values are likely to be chosen.

To circumvent this problem, as a possible remedy, one define a homotopy or deformation:

$$
\begin{gathered}
H: \mathbb{R}^{N} \times \mathbb{R} \rightarrow \mathbb{R}^{N} \text { such that } \\
H(u, \lambda)=F(u)-\lambda F\left(u_{1}\right),
\end{gathered}
$$

and attempt to trace an implicitly defined curve $C(s) \in H^{-1}(0)$ from the starting point $\left(u_{1}, 1\right)$ to a solution point $(\bar{u}, 0)$. If this succeeds, then a zero point $\bar{u}$ of $F$ is obtained. The above deformation is the so-called global homotopy. Typically, one 
can choose a convex homotopy such as

$$
H(u, \lambda)=\lambda G(u)+(1-\lambda) F(u),
$$

where $G: \mathbb{R}^{N} \times \mathbb{R} \rightarrow \mathbb{R}^{N}$ is a (trivial) smooth map having known zero point. Our case corresponds to the following deformation

$$
H(u, \lambda)=\frac{u}{\lambda}+F(u)
$$

In this case, we attempt to trace an implicitly defined curve $C(s) \in H^{-1}(0)$ from starting point $\left(u_{1}, \lambda_{1}\right)$ to a solution point $(\bar{u}, \lambda=\infty)$. Generally, it is sufficient to require some boundary conditions which essentially prevent the curve from running to infinity before intersecting the homotopy level $\lambda=\infty$, or returning back to level $\lambda=\lambda_{1}$, see figure 1 . The primary adavantage of this deformation method is to go progressively to the solution $\bar{u}$. Besides, it can overcome the numerical unstabilities arising in the standard Newton-Raphson's methods when we deal with a strong curvature or when we are near limit or bifurcation points. The map $\mathrm{H}$ introduced as a deformation, could equally well have arisen as an arbitrary underdetermined system of equations. Typical sources of such problems are discretizations of operator equations involving a natural parameter $\lambda$, e.g. boundary value problems, integral equations, ... etc.

In our case, as we will see, the time step $\delta t$ is in relation with the parameter $\lambda$. In the next sections, we will show how we define such deformation map $\mathrm{H}$ for our physical problem and how we can numerically trace the curve $\mathrm{C}(\mathrm{s})$ via the arclength predictor-corrector continuation methods.

We consider a system of three equations in a bounded domain $\Omega \subset \mathbb{R}^{2}$. This system forms the basic Drift-Diffusion model of mobile carrier transport in a semiconductor devices [6], [7], [8], [9], [10], [11]. The boundary conditions are mixed, and correspond to applied potential differences on the contact portions of the device (ohmic contacts) and to insulation in the remainder. So, we include the non homogeneous Dirichlet conditions for $\phi, \phi_{n}$ and $\phi_{p}$ on the ohmic contacts and the homogeneous Neumann conditions on insulating boundaries. The complete system, then, involves a coupled set of three strongly nonlinear partial differential equations, with their boundary conditions. We have to solve the following stationary boundary value problem with variable coefficients : 


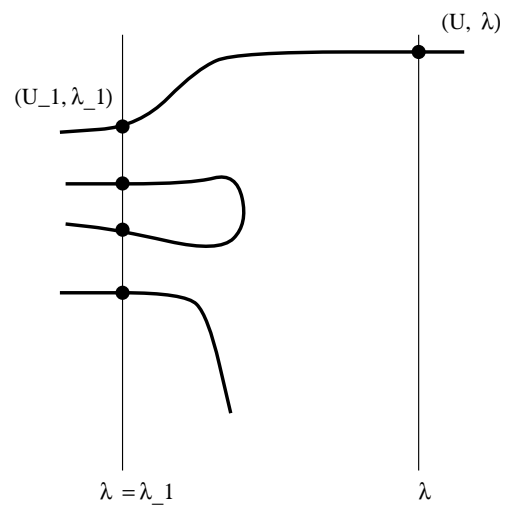

Figure 1: Different possibilities for the curve $C(s)$.

$$
\left\{\begin{aligned}
&-\operatorname{div}\left(\varepsilon_{r}(x) \nabla \phi\right)+\frac{q}{\varepsilon_{0}}\left(N\left(x, \phi, \phi_{n}\right)-P\left(x, \phi, \phi_{p}\right)-d_{o p}(x)\right)=0 \text { in } \Omega, \\
&-\operatorname{div}\left(q \mu_{n}(x) N\left(x, \phi, \phi_{n}\right) \nabla \phi_{n}\right)+q G R\left(x, \phi, \phi_{n}, \phi_{p}\right)=0 \text { in } \Omega, \\
&-\operatorname{div}\left(q \mu_{p}(x) P\left(x, \phi, \phi_{p}\right) \nabla \phi_{p}\right)-q G R\left(x, \phi, \phi_{n}, \phi_{p}\right)=0 \text { in } \Omega, \\
& \phi=g_{1}, \phi_{n}=\phi_{p}=g_{2} \quad \text { on } \quad \partial \Omega_{D}, \\
& \frac{\partial \phi}{\partial n}=\frac{\partial \phi_{n}}{\partial n}=\frac{\partial \phi_{p}}{\partial n}=0 \quad \text { on } \quad \partial \Omega_{N} .
\end{aligned}\right.
$$

The unknowns are the electrostatic potential $\phi$ and the quasi_Fermi levels $\phi_{n}$ and $\phi_{p}$ corresponding to the electron and hole concentrations. This choice of unknowns is well adapted for a numerical solution, since, $\phi, \phi_{n}$ and $\phi_{p}$ are continuous even for the heterojunction case, and the value assumed by these variables are of the same order as the applied potential. Here $n$ is the outward unit normal vector to the boundary $\partial \Omega$, and $\partial \Omega_{N}$ and $\partial \Omega_{D}$ are disjoint parts of $\partial \Omega$ such that $\partial \Omega_{N} \cup \partial \Omega_{D}=\partial \Omega$, where $\partial \Omega_{D}$ is of strictly positive measure in $\partial \Omega$. The function $\varepsilon_{r}$ is the semiconductor relative dielectric permittivity, $\varepsilon_{0}$ is the permittivity of vacuum, $N$ and $P$ are 
respectively the electron and hole concentrations. Typical models for these strongly nonlinear functions can be found in [10], [8], [12], [13], as well as for the mobilities $\mu_{n}$ and $\mu_{p}$. Moreover, the function $d_{o p}$ describes the doping profile, and is equal to $N_{D}-N_{A}, N_{D}$ and $N_{A}$ being the donor and acceptor impurity densities. The function GR is the so-called generation-recombinaison term which takes into account the possible 'birth' and 'death' of positive and negative charges. The model used for this term is the Shockley-Read-Hall one [8]. The constant q is the electron charge. The first equation of the above system is the nonlinear Poisson's equation for the potential. It corresponds to a semi-linear operator under divergence form. The second and the third equations are the transport equations with variable coefficients for negative and positive current densities $j_{n}, j_{p}$. These correspond to quasi-linear operators also under divergence form. In the heterojunction case, the coefficients are discontinuous. For additional details we refer to [7], [10], [13].

To approximate the solution we use the mixed finite element (MFE) methods [14], [15]. The main feature of this mixed formulation is that the electric displacement $D$ and the current densities $j_{n}$ and $j_{p}$ for electrons and holes, are taken as unknowns, together with the potential $\phi$ and quasi-Fermi levels $\phi_{n}$ and $\phi_{p}$. This allows $D, j_{n}$, and $j_{p}$ to be determined directly and accurately.

Another advantage of these approximations is to be current-preserving. This property is particularly important in the applications, as the current is possibly the most relevant unknown of the problem. Recently, in SISDEP conference in Bologne, Polack [16] has shown, for a model problem, that in (MFE) methods the currents are more accurate than in the case of Box-method. So, the (MFE) method seems to be attractive, for semiconductor numerical solution. Besides, it has a rigorous mathematical framework and good references in other disciplines such as fluid, solid mechanics [15], [17], or elasticity problems [18].

Existence results for the stationary problem hold under fairly general assumptions [19], [7], [6], [20], while uniqueness is not true in general. It holds only in some special cases [7], [6], [19], [21], [22], [20].

An outline of the paper is as follows. In section 2 we introduce the dual mixed variational formulation, and the system is decoupled by using a fixed-point strategy . We discuss, next the choice of functional spaces in the continuous and discrete cases. In section 3, some basic concepts of the continuation method are given. In section 4 we describe in detail the implementation of a continuation algorithm and one of its variant. In section 5 we present briefly some strategies for the steplength adaptation. Section 6 is devoted to the presentation of numerical results for realistic devices. 


\section{Mixed variational formulation in $H(\operatorname{div}, \Omega) \times L^{2}(\Omega)$}

\subsection{Mixed formulation}

The basic idea in the mixed formulation of (3) is to introduce with the solution $\left(\phi, \phi_{n}, \phi_{p}\right)$ of (3), the displacement $\mathrm{D}$, and the current densities $j_{n}, j_{p}$ as independent variables, satisfying the additional equations

$$
\begin{gathered}
D=\varepsilon_{r}(x) \nabla \phi, \\
j_{n}=q \mu_{n}(x) N\left(x, \phi, \phi_{n}\right) \nabla \phi_{n}, \\
j_{p}=q \mu_{p}(x) P\left(x, \phi, \phi_{p}\right) \nabla \phi_{p},
\end{gathered}
$$

and to derive a suitable variational formulation. The variables $\mathrm{D}, j_{n}$, and $j_{p}$ are the so-called dual unknowns. From a mathematical point of view, the mixed methods split the second-order problem (3) into two simpler first-order problems. From a physical viewpoint, they garantee an accurate determination of the dual unknowns. The fixed-point procedure applied to decouple the mixed system is then as follows. For $\phi_{0}, \phi_{n 0}, \phi_{p 0}$ given in some Banach spaces, we have to solve in parallel the mixed problems:

$$
\left\{\begin{array}{l}
-\operatorname{div}(D)+\frac{q}{\varepsilon_{0}}\left(N\left(x, \phi, \phi_{n 0}\right)-P\left(x, \phi, \phi_{p 0}\right)-d_{o p}(x)\right)=0 \quad \text { in } \Omega, \\
D=\varepsilon_{r}(x) \nabla \phi \quad \text { in } \quad \Omega, \\
-\operatorname{div}\left(j_{n}\right)+q G R\left(x, \phi_{0}, \phi_{n}, \phi_{p 0}\right)=0 \quad \text { in } \quad \Omega, \\
j_{n}=q \mu_{n}(x) N\left(x, \phi_{0}, \phi_{n}\right) \nabla \phi_{n} \quad \text { in } \quad \Omega, \\
-\operatorname{div}\left(j_{p}\right)-q G R\left(x, \phi_{0}, \phi_{n 0}, \phi_{p}\right)=0 \quad \text { in } \quad \Omega, \\
j_{p}=q \mu_{p}(x) P\left(x, \phi_{0}, \phi_{p}\right) \nabla \phi_{p} \quad \text { in } \quad \Omega, \\
\phi=g_{1}, \phi_{n}=\phi_{p}=g_{2} \quad \text { on } \quad \partial \Omega_{D}, \\
D . n=j_{n} . n=j_{p} . n=0 \quad \text { on } \quad \partial \Omega_{N} .
\end{array}\right.
$$

For mathematical purposes, we introduce the following notations :

$\operatorname{RR} \mathrm{n}^{\circ} 2546$ 


$$
\begin{aligned}
& a_{1}(x)=\varepsilon_{r}(x), \quad \alpha_{1}=a_{1}^{-1}, \\
& a_{2}\left(x, \phi, \phi_{n}\right)=q \mu_{n}(x) N\left(x, \phi_{0}, \phi_{n}\right), \quad \alpha_{2}=a_{2}^{-1}, \\
& a_{3}\left(x, \phi, \phi_{p}\right)=q \mu_{p}(x) P\left(x, \phi_{0}, \phi_{p}\right), \quad \alpha_{3}=a_{3}^{-1}, \\
& c_{1}\left(x, \phi, \phi_{n}, \phi_{p}\right)=\frac{q}{\varepsilon_{0}}\left(N\left(x, \phi, \phi_{n 0}\right)-P\left(x, \phi, \phi_{p 0}\right)-d_{o p}(x)\right), \\
& c_{2}\left(x, \phi, \phi_{n}, \phi_{p}\right)=q G R\left(x, \phi_{0}, \phi_{n}, \phi_{p 0}\right), \\
& c_{3}\left(x, \phi, \phi_{n}, \phi_{p}\right)=-q G R\left(x, \phi_{0}, \phi_{n 0}, \phi_{p}\right),
\end{aligned}
$$

Remark 2.1 The mixed formulation (or hybrid formulation ) [23] is particularly adapted to devices composed of many materials ( HBT for example) and having irregularly shaped interfaces.

\subsection{Dual mixed variational formulation in the continuous case}

The nonstandard dual mixed formulation of (7) is:

Find $\left(\phi, D ; \phi_{n}, j_{n} ; \phi_{p}, j_{p}\right) \in\left[W \times V_{0}\right]^{3}$ such that:

$$
\begin{gathered}
\int_{\Omega} \alpha_{1}(x) D v_{1} d x+\int_{\Omega} \phi \operatorname{div}\left(v_{1}\right) d x=\left\langle g_{1}, v_{1} . n\right\rangle \forall v_{1} \in V_{0}, \\
-\int_{\Omega} \operatorname{div}(D) w_{1} d x+\int_{\Omega} c_{1}\left(x, \phi, \phi_{n}, \phi_{p}\right) w_{1} d x=0 \quad \forall w_{1} \in W, \\
\int_{\Omega} \alpha_{2}\left(x, \phi, \phi_{n}\right) j_{n} v_{2} d x+\int_{\Omega} \phi_{n} \operatorname{div}\left(v_{2}\right) d x=\left\langle g_{2}, v_{2} . n\right\rangle \quad \forall v_{2} \in V_{0}, \\
-\int_{\Omega} \operatorname{div}\left(j_{n}\right) w_{2} d x+\int_{\Omega} c_{2}\left(x, \phi, \phi_{n}, \phi_{p}\right) w_{2} d x=0 \quad \forall w_{2} \in W,
\end{gathered}
$$




$$
\begin{aligned}
& \int_{\Omega} \alpha_{3}\left(x, \phi, \phi_{p}\right) j_{p} v_{3} d x+\int_{\Omega} \phi_{p} \operatorname{div}\left(v_{3}\right) d x=\left\langle g_{2}, v_{3} . n\right\rangle \forall v_{3} \in V_{0}, \\
& -\int_{\Omega} \operatorname{div}\left(j_{p}\right) w_{3} d x+\int_{\Omega} c_{3}\left(x, \phi, \phi_{n}, \phi_{p}\right) w_{3} d x=0 \quad \forall w_{3} \in W
\end{aligned}
$$

where $V_{0}$ and $\mathrm{W}$ are two functional spaces to be determined. In order to garantee existence and uniqueness for each of sub-systems (9), (10), and (11), the spaces $V_{0}$ and $\mathrm{W}$ must be properly related, see Girault-Raviart [15], [23]. The basic choice is

$$
\begin{gathered}
V=H(\operatorname{div}, \Omega)=\left\{v \in\left[L^{2}(\Omega)\right]^{2} / \operatorname{div}(v) \in L^{2}(\Omega)\right\}, \\
V_{00}=\left\{v \in C^{0}(\bar{\Omega})^{2} /\left.v \cdot n\right|_{\partial \Omega}=0 \text { on } \partial \Omega_{N}\right\}, \\
V_{0}=\bar{V}_{00}^{V}
\end{gathered}
$$

so that $V_{0}$ is the closure of $V_{00}$ for the topology of $\mathrm{V}$. Hence, $V_{0}$ is closed in $\mathrm{V}$, then it is a Hilbert space. The norm in $\mathrm{V}$ is simply

$$
\|v\|_{V}^{2}=\|v\|_{0}^{2}+\|\operatorname{div}(v)\|_{0}^{2}
$$

where $\|.\|_{0}$ is the usal norm in $L^{2}(\Omega),\left[L^{2}(\Omega)\right]^{2}$ or $L^{2}(\partial \Omega)$. Throughout the paper, (.,.) denotes the scalar product in $L^{2}(\Omega)$, and $\langle.,$.$\rangle denotes the duality product bet-$ ween $H^{\frac{1}{2}}(\partial \Omega)$ and $H^{-\frac{1}{2}}(\partial \Omega)$.

In addition, we choose

$$
W=L^{2}(\Omega) .
$$

Remark 2.2 In the dual mixed variational formulation the space $V_{0}$ of dual variables is more regular than the space $W$ of primal variables. In the primal version, the choice would be $V_{0}=\left[L^{2}(\Omega)\right]^{2}$, and $W$ a subset of $H^{1}(\Omega)$.

\subsection{Discretization of the mixed formulation}

When dealing with discretizations of a mixed formulation, proper relations between the discrete analogous $V_{0 h}$ and $W_{h}$ have to be satisfied in order to garantee existence and uniqueness of the discrete solutions, and optimal error bounds [23]. In our case, we consider a conforming approximation which implies $W_{h} \subset W, V_{0 h} \subset V_{0}$. While the requirement $W_{h} \subset W=L^{2}(\Omega)$ does not pose any problem, the inclusion $V_{0 h} \subset V_{0}$ implies some regularity on the elements of $V_{0 h}$ : more precisely, the normal 
components of a vector in $V_{0 h}$ must be continuous across the interelement boundaries. For our problem, this property will garantee the numerical conservation of the current.

In the literature, there are four families of mixed finite elements [24], [14], [25] satisfying all the requirements and giving optimal error bounds. The key properties of these families are their conformity (i.e. $W_{h} \subset W, V_{0 h} \subset V_{0}$ ) and the commutativity of the divergence operator with suitably defined 'projections' from the continuous spaces onto the discrete ones [25], [49], [24], [23].

Suppose for simplicity that $\Omega$ is a polygonal domain and let $\mathcal{T}_{h}$ be a regular family of triangulations of $\Omega\left(\Omega=\cup_{T \in \mathcal{T}_{h}} T\right)$. A suitable approximation of problems (9), (10), and (11) can be obtained by the Raviart-Thomas elements of lowest degree [14], [23], [15].

The elements of $\mathrm{W}$ are approximated by piecewise constant functions (no continuity requirements), while the vectors of $V_{0}$ are approximated by piecewise linear polynomials whose normal components are constant and continuous across edges. Therefore, we define :

$$
\begin{gathered}
V_{h}=\left\{v \in V /\left.v\right|_{T} \in R T_{0}(T), \forall T \in \mathcal{T}_{h}\right\}, \\
V_{0 h}=V_{0} \cap V_{0 h}, \\
W_{h}=\left\{w \in L^{2}(\Omega) /\left.w\right|_{T} \in P_{0}(T), \forall T \in \mathcal{T}_{h}\right\},
\end{gathered}
$$

where for all triangle $T \in \mathcal{T}_{h}$, we have

$$
R T_{0}(T)=\left\{v=\left(v_{1}, v_{2}\right)=(\alpha+\gamma x, \beta+\gamma y), \alpha, \beta, \gamma \in \mathbb{R}\right\},
$$

and $P_{0}(T)$ is a space of constant polynomials defined on $\mathrm{T}$.

From the definitions given above, it is clear that $V_{h}, V_{0 h}, W_{h}$ are finite dimensional spaces.

Let NE be the number of triangles $T \in \mathcal{T}_{h}$, NA the number of all edges of $\mathcal{T}_{h}$, and $\mathrm{NAB}$ the number of edges located on the Neumann part $\partial \Omega_{N}$. Setting NN $=$ NA $\mathrm{NAB}$, we have

$$
\operatorname{dim}\left(V_{h}\right)=N A, \operatorname{dim}\left(V_{0 h}\right)=N N, \operatorname{dim}\left(W_{h}\right)=N E .
$$

\subsection{Degrees of freedom of $V_{h}$ and mixed discretization}

To approximate $H(\operatorname{div}, \Omega)$ by $R T_{0}(T)$, it is necessary to have continuity of the normal components of the vectors of $V_{h}$ across the interelement boundaries. This is 
garanteed by an appropriate choice of degrees of freedom.

For the lowest order Raviart-Thomas element, we associate one degree of freedom to each edge of the triangle determining the value of the component of $\mathrm{v}$ normal to this edge, see figure 2.

The mixed discretization of our problem is defined as follows :

Find $\left(\phi_{h}, D_{h} ; \phi_{n h}, j_{n h} ; \phi_{p h}, j_{p h}\right) \in\left[W_{h} \times V_{0 h}\right]^{3}$ such that :

$$
\begin{aligned}
& \left\{\begin{array}{l}
\int_{\Omega} \alpha_{1}(x) D_{h} v_{1} d x+\int_{\Omega} \phi_{h} \operatorname{div}\left(v_{1}\right) d x=\left\langle g_{1}, v_{1} . n\right\rangle \forall v_{1} \in V_{0 h}, \\
-\int_{\Omega} \operatorname{div}\left(D_{h}\right) w_{1} d x+\int_{\Omega} c_{1}\left(x, \phi_{h}, \phi_{n h}, \phi_{p h}\right) w_{1} d x=0 \forall w_{1} \in W_{h},
\end{array}\right. \\
& \left\{\begin{array}{l}
\int_{\Omega} \alpha_{2}\left(x, \phi_{h}, \phi_{n h}\right) j_{n h} v_{2} d x+\int_{\Omega} \phi_{n h} \operatorname{div}\left(v_{2}\right) d x=\left\langle g_{2}, v_{2} . n\right\rangle \forall v_{2} \in V_{0 h}, \\
-\int_{\Omega} \operatorname{div}\left(j_{n h}\right) w_{2} d x+\int_{\Omega} c_{2}\left(x, \phi_{h}, \phi_{n h}, \phi_{p h}\right) w_{2} d x=0 \forall w_{2} \in W_{h},
\end{array}\right. \\
& \left\{\begin{array}{l}
\int_{\Omega} \alpha_{3}\left(x, \phi_{h}, \phi_{p h}\right) j_{p h} v_{3} d x+\int_{\Omega} \phi_{p h} \operatorname{div}\left(v_{3}\right) d x=\left\langle g_{2}, v_{3} . n\right\rangle \forall v_{3} \in V_{0 h}, \\
-\int_{\Omega} \operatorname{div}\left(j_{p h}\right) w_{3} d x+\int_{\Omega} c_{3}\left(x, \phi_{h}, \phi_{n h}, \phi_{p h}\right) w_{3} d x=0 \forall w_{3} \in W_{h} .
\end{array}\right.
\end{aligned}
$$

Remark 2.3 This mixed problem is nonstandard, since the functions $\alpha_{2,3}$, and $c_{1,2,3}$ are nonlinear.

When dealing with parametric systems (12), (13), and (14) from a numerical point of view, one key point is the choice of iterative methods to compute the solution. We recall that the most used iterative algorithms are variants of the Newton-Raphson's method [26], [27] . The quadratic convergence of the Newton-Raphson's method is obtained only if we are in a neighborhood of the desired solution. Therfore, the choice of the initial guess is critical. In the applications, very little a priori knowledge concerning the solution is available. So, the classical Newton-Raphson's method may fail, because poor starting values are likely to be chosen. As a possible remedy, we use the time deformation. The basic idea of this latter is to introduce an artificial transient problem. Hence, instead of computing directly the final solution, we 


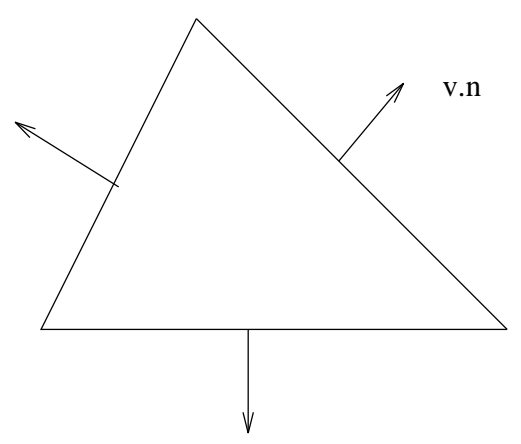

a) $\mathrm{RTO}(\mathrm{T})$

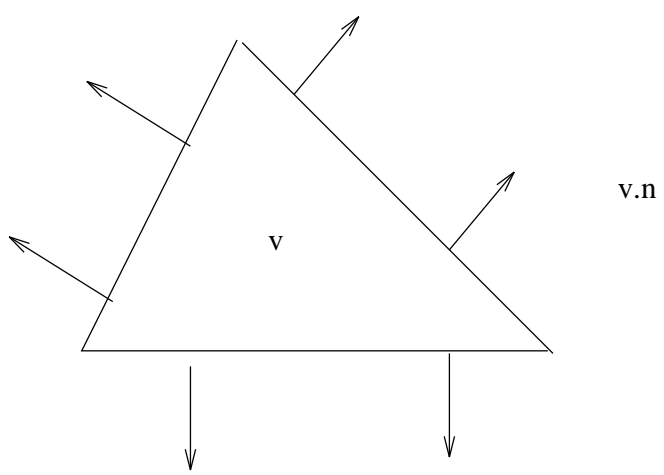

b) RT1(T)

Figure 2: Raviart-Thomas mixed finite elements of low order.

construct it step by step. This computing strategy provides a "good" initial guess. The algebraic solution of our problem is then obtained as the limit solution of the following problem.

Find $\left(\phi, D, \phi_{n}, j_{n}, \phi_{p}, j_{p}\right):\{\Omega \times] 0, L[\}^{6} \rightarrow \mathbb{R}$ such that : 


$$
\left\{\begin{array}{l}
\left.A_{1}(x) \frac{\partial \phi}{\partial t}-\operatorname{div}(D)+c_{1}\left(x, \phi, \phi_{n}, \phi_{p}\right)=0 \text { in } \Omega \times\right] 0, L[, \\
\left.D=\alpha_{1}(x) \nabla \phi \quad \text { in } \Omega \times\right] 0, L[, \\
\left.A_{2}(x) \frac{\partial \phi_{n}}{\partial t}-\operatorname{div}\left(j_{n}\right)+c_{2}\left(x, \phi, \phi_{n}, \phi_{p}\right)=0 \text { in } \Omega \times\right] 0, L[, \\
\left.j_{n}=\alpha_{2}\left(x, \phi, \phi_{n}\right) \nabla \phi_{n} \quad \text { in } \Omega \times\right] 0, L[, \\
\left.A_{3}(x) \frac{\partial \phi_{p}}{\partial t}-\operatorname{div}\left(j_{p}\right)+c_{3}\left(x, \phi, \phi_{n}, \phi_{p}\right)=0 \text { in } \Omega \times\right] 0, L[, \\
\left.j_{p}=\alpha_{3}\left(x, \phi, \phi_{p}\right) \nabla \phi_{p} \quad \text { in } \Omega \times\right] 0, L[, \\
\left.\phi=g_{1}, \quad \phi_{n}=\phi_{p}=g_{2} \quad \text { on } \partial \Omega_{D} \times\right] 0, L[, \\
\left.D . n=j_{n} . n=j_{p} . n=0 \quad \text { on } \partial \Omega_{N} \times\right] 0, L[, \\
\left(\phi(x, 0), \phi_{n}(x, 0), \phi_{p}(x, 0)=\left(\phi^{0}, \phi_{n}^{0}, \phi_{p}^{0}\right) \quad \text { in } \Omega,\right. \\
\left(D(x, 0), j_{n}(x, 0), j_{p}(x, 0)\right)=\left(D^{0}, j_{n}^{0}, j_{p}^{0}\right) \quad \text { in } \Omega,
\end{array}\right.
$$

where $A_{1,2,3}$ are linear, positive functions used as preconditioners. They are assumed to be uniformally bounded. Setting

$$
A_{1,2,3}^{0} \leq A_{1,2,3} \leq A_{1,2,3}^{1} .
$$

Here, the time parameter L is strictly positive.

\subsection{Time discretization}

We consider successively each pair of equations and apply a nonlinear implicit scheme with local time steps [28]. The non major unknowns, i.e. $\phi_{n}$ and $\phi_{p}$ for the Poisson's equation, for example, are considered as parameters which take the latest known values. A substantial advantage of this kind of (relaxation) scheme is to be inexpensive either in space or in time. Besides, it leads to modular codes in which other equations can be added to the Drift-Diffusion model (as energy conservation equation or moment conservation equation for example), without changing the basic structure 
of the code.

For all $k \geq 0, \phi^{k}, \phi_{n}^{k}, \phi_{p}^{k}, D^{k}, j_{n}^{k}$, and $j_{p}^{k}$ being given, we therefore solve the following mixed problem :

Find $\left(\phi^{k+1}, D^{k+1}, \phi_{n}^{k+1}, j_{n}^{k+1}, \phi_{p}^{k+1}, j_{p}^{k+1}\right)$ such that :

$$
\left\{\begin{array}{l}
A_{1}(x) \frac{\left(\phi^{k+1}-\phi^{k}\right)}{\delta t_{1}}-\operatorname{div}\left(D^{k+1}\right)+c_{1}\left(x, \phi^{k+1}, \phi_{n}^{k}, \phi_{p}^{k}\right)=0 \quad \text { in } \Omega, \\
D^{k+1}=\alpha_{1}(x) \nabla \phi^{k+1} \quad \text { in } \Omega, \\
A_{2}(x) \frac{\left(\phi_{n}^{k+1}-\phi_{n}^{k}\right)}{\delta t_{2}}-\operatorname{div}\left(j_{n}^{k+1}\right)+c_{2}\left(x, \phi^{k+1}, \phi_{n}^{k+1}, \phi_{p}^{k}\right)=0 \quad \text { in } \Omega, \\
j_{n}^{k+1}=\alpha_{2}\left(x, \phi^{k+1}, \phi_{n}^{k+1}\right) \nabla \phi_{n}^{k+1} \quad \text { in } \Omega, \\
A_{3}(x) \frac{\left(\phi_{p}^{k+1}-\phi_{p}^{k}\right)}{\delta t_{3}}-\operatorname{div}\left(j_{p}^{k+1}\right)+c_{3}\left(x, \phi^{k+1}, \phi_{n}^{k+1}, \phi_{p}^{k+1}\right)=0 \quad \text { in } \Omega, \\
j_{p}^{k+1}=\alpha_{3}\left(x, \phi^{k+1}, \phi_{p}^{k+1}\right) \nabla \phi_{p}^{k+1} \quad \text { in } \Omega, \\
\phi^{k+1}=g_{1}, \quad \phi_{n}^{k+1}=\phi_{p}^{k+1}=g_{2} \text { on } \partial \Omega_{D}, \\
D^{k+1} . n=j_{n}^{k+1} . n=j_{p}^{k+1} . n=0 \text { on } \partial \Omega_{N} .
\end{array}\right.
$$

The functions $A_{1,2,3}$ introduce the local time steps

$$
\delta t^{1,2,3}(x)=A_{1,2,3}^{-1}(x) \delta t_{1,2,3} .
$$

The time steps $\delta t_{1,2,3}$ are looked here as dimensionless parameters (the time dimension is introduced in $A_{1,2,3}^{-1}$ ) which will be used for the adaptation of local time steps $\delta t^{1,2,3}(x)$.

From our numerical results, it seems that these nonlinear implicit schemes with local time steps are robust and have good numerical properties : Convergence, stabiltiy, and consistency.

Because of the nonlinearity, the convergence of the above algorithm depends on the choice of local time steps and their adaptation to the nonlinarity of the problem. 
In choosing the time steps, one has to compromise between computing cost and convergence speed. If a small time step is chosen then the number of time steps to obtain the stationary solution will increase. In the contrary, if too large time steps are considered, then the Newton-Raphson's type algorithm may generate a divergent sequence. Hence, the choice of time steps can't be arbitrary.

We have taken into account various theoretical and numerical considerations to give some estimates of our local time steps (constant value on each triangle of the mesh will be taken). From the results given in [28], [29], we take for our numerical experiments and for all implicit steps, the following estimates :

\section{$\underline{\text { Poisson equation }}$}

$$
\delta t^{1}(k+1, T)=\delta t_{1}^{k+1} A_{1 T}^{-1}=\delta t_{1}^{k+1} \min \left(\frac{s}{\alpha_{1}(\bar{x})}, \frac{1}{c_{1, \phi}^{\prime}\left(\bar{x}, \phi^{k}, \phi_{n}^{k}, \phi_{p}^{k}\right)}\right) .
$$

\section{Continuity equation for electrons}

$$
\delta t^{2}(k+1, T)=\delta t_{2}^{k+1} A_{2 T}^{-1}=\delta t_{2}^{k+1} \min \left(\frac{s}{\alpha_{2}\left(\bar{x}, \phi^{k+1}, \phi_{n}^{k}\right)}, \frac{1}{c_{2, \phi_{n}}^{\prime}\left(\bar{x}, \phi^{k+1}, \phi_{n}^{k}, \phi_{p}^{k}\right)}\right) .
$$

\section{Continuity equation for holes}

$$
\delta t^{3}(k+1, T)=\delta t_{3}^{k+1} A_{3 T}^{-1}=\delta t_{3}^{k+1} \min \left(\frac{s}{\alpha_{3}\left(\bar{x}, \phi^{k+1}, \phi_{p}^{k}\right)}, \frac{1}{c_{3, \phi_{p}}^{\prime}\left(\bar{x}, \phi^{k+1}, \phi_{n}^{k+1}, \phi_{p}^{k}\right)}\right) .
$$

The value of $\bar{x}$ can be the barycenter of element T.

To solve numerically the above decoupled mixed system with

$$
\left(\phi^{k+1}, D^{k+1}, \phi_{n}^{k+1}, j_{n}^{k+1}, \phi_{p}^{k+1}, j_{p}^{k+1}\right)
$$

as unknowns, we approximate it by the (MFE) method described above.

Since our (MFE) approximation is of lowest order, the primal variables $\phi, \phi_{n}$, and $\phi_{p}$ are constant by element. This implies that the time steps $\delta t^{1,2,3}$ are easily computed. This leads to a nonlinear parametric systems with a finite number of unknowns. The discrete mixed variational formulation is as follows.

Find $\left(\phi_{h}^{k+1}, D_{h}^{k+1}, \phi_{n h}^{k+1}, j_{n h}^{k+1}, \phi_{p h}^{k+1}, j_{p h}^{k+1}\right) \in W_{h} \times V_{0 h}$ such that : 


$$
\begin{aligned}
& \left\{\begin{array}{l}
\int_{\Omega} \alpha_{1}(x) D_{h}^{k+1} v_{h} d x+\int_{\Omega} \operatorname{div}\left(v_{h}\right) \phi_{h}^{k+1} d x=\left\langle g_{1}, v_{h} . n\right\rangle \quad \forall v_{h} \in V_{0 h}, \\
-\int_{\Omega} \operatorname{div}\left(D_{h}^{k+1}\right) w_{h} d x+\int_{\Omega}\left[\frac{A_{1}}{\delta t_{1}^{k+1}} \phi_{h}^{k+1}+c_{1}\left(x, \phi_{h}^{k+1}, \phi_{n h}^{k}, \phi_{p h}^{k}\right)\right] \cdot w_{h} d x= \\
\int_{\Omega} \frac{A_{1}}{\delta t_{1}^{k+1}} \phi_{h}^{k} w_{h} d x \forall w_{h} \in W_{h},
\end{array}\right. \\
& \left\{\begin{array}{l}
\int_{\Omega} \alpha_{2}\left(x, \phi_{h}^{k+1}, \phi_{n h}^{k+1}\right) j_{n h}^{k+1} v_{h} d x+\int_{\Omega} \operatorname{div}\left(v_{h}\right) \phi_{n h}^{k+1} d x=\left\langle g_{2}, v_{h} . n\right\rangle \quad \forall v_{h} \in V_{0 h}, \\
-\int_{\Omega} \operatorname{div}\left(j_{n h}^{k+1}\right) w_{h} d x+\int_{\Omega}\left[\frac{A_{2}}{\delta t_{2}^{k+1}} \phi_{n h}^{k+1}+c_{2}\left(x, \phi_{n h}^{k+1}, \phi_{n h}^{k+1}, \phi_{p h}^{k}\right)\right] w_{h} d x= \\
\int_{\Omega} \frac{A_{2}}{\delta t_{2}^{k+1}} \phi_{n h}^{k} w_{h} d x \forall w_{h} \in W_{h},
\end{array}\right. \\
& \left\{\begin{array}{l}
\int_{\Omega} \alpha_{3}\left(x, \phi_{h}^{k+1}, \phi_{p h}^{k+1}\right) j_{p h}^{k+1} v_{h} d x+\int_{\Omega} \operatorname{div}\left(v_{h}\right) \phi_{p h}^{k+1} d x=\left\langle g_{2}, v_{h} . n\right\rangle \quad \forall v_{h} \in V_{0 h}, \\
-\int_{\Omega} \operatorname{div}\left(j_{p h}^{k+1}\right) w_{h} d x+\int_{\Omega}\left[\frac{A_{3}}{\delta t_{3}^{k+1}} \phi_{p h}^{k+1}+c_{3}\left(x, \phi_{h}^{k+1}, \phi_{n h}^{k+1}, \phi_{p h}^{k+1}\right)\right] w_{h} d x= \\
\int_{\Omega} \frac{A_{3}}{\delta t_{3}^{k+1}} \phi_{p h}^{k} w_{h} d x \forall w_{h} \in W_{h} .
\end{array}\right.
\end{aligned}
$$

Since the functions $A_{1,2,3}$ are assumed to be bounded, one can use the existence and uniqueness results derived in [29], [30], [31] to prove that each of sub-systems (17), (18), or (19) has a unique solution.

For the sake of the simplicity the index $\mathrm{h}$ will be omitted. Setting

$$
\bar{\lambda}_{1,2,3}^{k+1}=\delta t_{1,2,3}^{k+1},
$$

we introduce the dimensionless time $\lambda_{1,2,3}^{k+1}$ by the (trivial) relation

$$
\lambda_{1,2,3}^{k+1}-\lambda_{1,2,3}^{k}=\bar{\lambda}_{1,2,3}^{k+1} .
$$


The problem to solve can be reformulated as follows. For each $k \geq 0$ and for all parameters $\lambda_{1,2,3}^{k+1}$ given, we have to solve :

Find $\left(\phi^{k+1}, D^{k+1}, \phi_{n}^{k+1}, j_{n}^{k+1}, \phi_{p}^{k+1}, j_{p}^{k+1}\right) \in W_{h} \times V_{0 h}$ such that :

$$
\begin{gathered}
H_{1}\left(\phi^{k+1}, D^{k+1}, \lambda_{1}^{k+1}, g_{1}\right)=0, \\
H_{2}\left(\phi_{n}^{k+1}, j_{n}^{k+1}, \lambda_{2}^{k+1}, g_{2}\right)=0, \\
H_{3}\left(\phi_{p}^{k+1}, j_{p}^{k+1}, \lambda_{3}^{k+1}, g_{2}\right)=0 .
\end{gathered}
$$

The parameters here are $\lambda_{1,2,3}^{k+1}$, and the data $g_{1,2}$. The functions $H_{1,2,3}$ are given by the following equations :

$$
\begin{aligned}
& H_{1}=\left[\begin{array}{c}
H_{11, i} \\
H_{12, j+N N}
\end{array}\right] \quad \forall i=1, N N \quad \forall j=1, N E, \\
& H_{2}=\left[\begin{array}{c}
H_{21, i} \\
H_{22, j+N N}
\end{array}\right] \quad \forall i=1, N N \quad \forall j=1, N E, \\
& H_{3}=\left[\begin{array}{c}
H_{31, i} \\
H_{32, j+N N}
\end{array}\right] \quad \forall i=1, N N \quad \forall j=1, N E,
\end{aligned}
$$

with

$$
\begin{aligned}
& \left\{\begin{array}{l}
H_{11, i}\left(\phi^{k+1}, D^{k+1}, \lambda_{1}^{k+1}, g_{1}\right)=\int_{\Omega} \alpha_{1}(x) D^{k+1} v_{i} d x+\int_{\Omega} \phi^{k+1} d i v\left(v_{i}\right) d x- \\
\int_{\partial \Omega_{D}} g_{1} v_{i} . n d \sigma \quad \forall i=1, N N,
\end{array}\right. \\
& \left\{\begin{array}{l}
H_{12, i+N N}\left(\phi^{k+1}, D^{k+1}, \lambda_{1}^{k+1}, g_{1}\right)=-\int_{\Omega} \operatorname{div}\left(D^{k+1}\right) w_{i} d x+ \\
\int_{\Omega}\left[\frac{A_{1}}{\bar{\lambda}_{1}^{k+1}} \phi^{k+1}+c_{1}\left(x, \phi^{k+1}, \phi_{n}^{k+1}, \phi_{p}^{k+1}\right)\right] w_{i} d x-\int_{\Omega} \frac{A_{1}}{\bar{\lambda}_{1}^{k+1}} \phi^{k} w_{i} d x \forall i=1, N E
\end{array}\right. \\
& \left\{\begin{array}{l}
H_{21, i}\left(\phi_{n}^{k+1}, j_{n}^{k+1}, \lambda_{2}^{k+1}, g_{2}\right)=\int_{\Omega} \alpha_{2}\left(x, \phi^{k+1}, \phi_{n}^{k+1}\right) j_{n}^{k+1} v_{i} d x+\int_{\Omega} \operatorname{div}\left(v_{i}\right) \phi_{n}^{k+1} d x- \\
\int_{\partial \Omega_{D}} g_{2} v_{i} . n d \sigma \quad \forall i=1, N N
\end{array}\right.
\end{aligned}
$$




$$
\begin{aligned}
& \left\{\begin{array}{l}
H_{22, i+N N}\left(\phi_{n}^{k+1}, j_{n}^{k+1}, \lambda_{2}^{k+1}, g_{2}\right)=-\int_{\Omega} \operatorname{div}\left(j_{n}^{k+1}\right) w_{i} d x+ \\
\int_{\Omega}\left[\frac{A_{2}}{\bar{\lambda}_{2}^{k+1}} \phi_{n}^{k+1}+c_{2}\left(x, \phi^{k+1}, \phi_{n}^{k+1}, \phi_{p}^{k}\right)\right] w_{i} d x-\int_{\Omega} \frac{A_{2}}{\bar{\lambda}_{2}^{k+1}} \phi_{n}^{k} w_{i} d x \quad \forall i=1, N E
\end{array}\right. \\
& \left\{\begin{array}{l}
H_{31, i}\left(\phi_{p}^{k+1}, j_{p}^{k+1}, \lambda_{3}^{k+1}, g_{2}\right)=\int_{\Omega} \alpha_{3}\left(x, \phi^{k+1}, \phi_{p}^{k+1}\right) j_{p}^{k+1} v_{i} d x+\int_{\Omega} \operatorname{div}\left(v_{i}\right) \phi_{p}^{k+1} d x- \\
\int_{\partial \Omega_{D}} g_{2} v_{i} . n d \sigma \forall i=1, N N
\end{array}\right. \\
& \left\{\begin{array}{l}
H_{32, i+N N}\left(\phi_{p}^{k+1}, j_{p}^{k+1}, \lambda_{3}^{k+1}, g_{2}\right)=-\int_{\Omega} \operatorname{div}\left(j_{p}^{k+1}\right) w_{i} d x+ \\
\int_{\Omega}\left[\frac{A_{3}}{\bar{\lambda}_{3}^{k+1}} \phi_{p}^{k+1}+c_{3}\left(x, \phi^{k+1}, \phi_{n}^{k+1}, \phi_{n}^{k+1}\right)\right] w_{i} d x-\int_{\Omega} \frac{A_{3}}{\bar{\lambda}_{3}^{k+1}} \phi_{p}^{k} w_{i} d x \quad \forall i=1, N E .
\end{array}\right.
\end{aligned}
$$

Above, $v_{i}$ and $w_{i}$ are the nodal basis shape functions of the finite element spaces $V_{h}$ and $W_{h}$. The adaptation of local time steps is given by the adapation of the parameters $\lambda_{1,2,3}^{k+1}$ (or $\bar{\lambda}_{1,2,3}^{k+1}$ ). Some manual strategy are given in [28], [32]. The aim of this paper is to give an automatic and rigorous adaptation of these latter parameters. To this end, we propose the Arc-length continuation methods [33], [34], [35], [36], [37], [38], [39], [40].

\section{Basic concepts of the continuation method}

\subsection{Classical continuation method of Euler-Newton's type}

To give the basic concepts of the continuation methods, we study the first subsystem. The analysis of the other sub-systems is analogous. We set

$$
u=\left(\phi^{k+1}, D^{k+1}\right) \quad \lambda=\lambda_{1}^{k+1} .
$$

The solution curve $C_{1}$ of the first sub-system (20) can now be parametrized with respect to the parameter $\lambda$. Indeed, if for $\lambda=\lambda_{0}$ a solution $u=u^{0}$ of (20) is isolated, that is if

$$
H_{1, u}^{0}=\frac{\partial H_{1}}{\partial u}\left(u^{0}, \lambda^{0}\right)
$$


is nonsingular, and if $H_{1}(u, \lambda)$ is smooth in some $\rho_{0^{-}}$sphere about $\left[u^{0}, \lambda^{0}\right]$ then the Implicit Function Theorem ensures the existence of a unique smooth arc of solution $u=u(\lambda)$ for $\left|\lambda-\lambda^{0}\right|<\rho$, say. Furthermore, under smoothness assumptions, it follows that $\frac{d u(\lambda)}{d \lambda}$ exists and satifies :

$$
H_{1, u}(u(\lambda), \lambda) \frac{d u(\lambda)}{d \lambda}=-H_{1, \lambda}(u(\lambda), \lambda)
$$

The basic idea in continuation methods for approximating the solution curve $C_{1}$ from, say $\lambda=\lambda^{0}$ to $\lambda$ is explained in the following algorithm.

\subsection{Algorithm 1 (Embedding Algorithm) :}

Step 1. predict the solution $u^{0}(\lambda+\delta \lambda)$ by the explicit Euler scheme

$$
u^{0}(\lambda+\delta \lambda)=u(\lambda)+\delta \lambda \frac{d u(\lambda)}{d \lambda}
$$

Step 2. correct the first order prediction by solving

$$
H_{1, u}^{n} \delta u^{n}(\lambda+\delta \lambda)=-H_{1}^{n} \quad n=0,1,2, \ldots
$$

by a classical Newton-Raphson's method taking as initial gess the prediction. Here, we have

$$
\begin{gathered}
H_{1, u}^{n}=H_{1, u}\left(u^{n}(\lambda+\delta \lambda), \lambda+\delta \lambda\right), \\
H_{1}^{n}=H_{1}\left(u^{n}(\lambda+\delta \lambda), \lambda+\delta \lambda\right), \\
u^{n+1}(\lambda+\delta \lambda)=u^{n}(\lambda+\delta \lambda)+\delta u^{n}(\lambda+\delta \lambda) .
\end{gathered}
$$

Remark 3.1 The classical embedding methods have been extensively surveyed by Ficken (1951) [41], Wasserstrom (1973) [42] and Wacker (1978) [43]. In the engineering community, the above continuation algorithm, see figure 3 is also called incremental loading algorithm [44].

The idea behind the above algorithm is clear : if the increment $\delta \lambda$ is chosen sufficiently small, then the iterative process will generaly converge since the starting value $u^{0}$ will be close to the solution.

The drawback of this method is that it will fail when turning points of the curve with respect to $\lambda$ are encountered, see figures 4, 5 (this situation may occur for some semiconductor devices, and when doing continuation on applied potentials).

$\mathrm{RR} \mathrm{n}^{\circ} 2546$ 
However, when the nonlinearity is strong (as in our cases), see figures $6,7,8$, it may be necessary to choose an extremely small increment $\delta \lambda$ in order for the above algorithm to succeed.

The failure or poor performance of the above continuation algorithm can be attributed to the fact that the parameter $\lambda$ may be ill suited as a parametrization for the curve.

One remedy is to change the parametrization and to take the arclength as a naturel parameter for the curve. The parameter $\lambda$ will be taken now as unknown together with $u$.

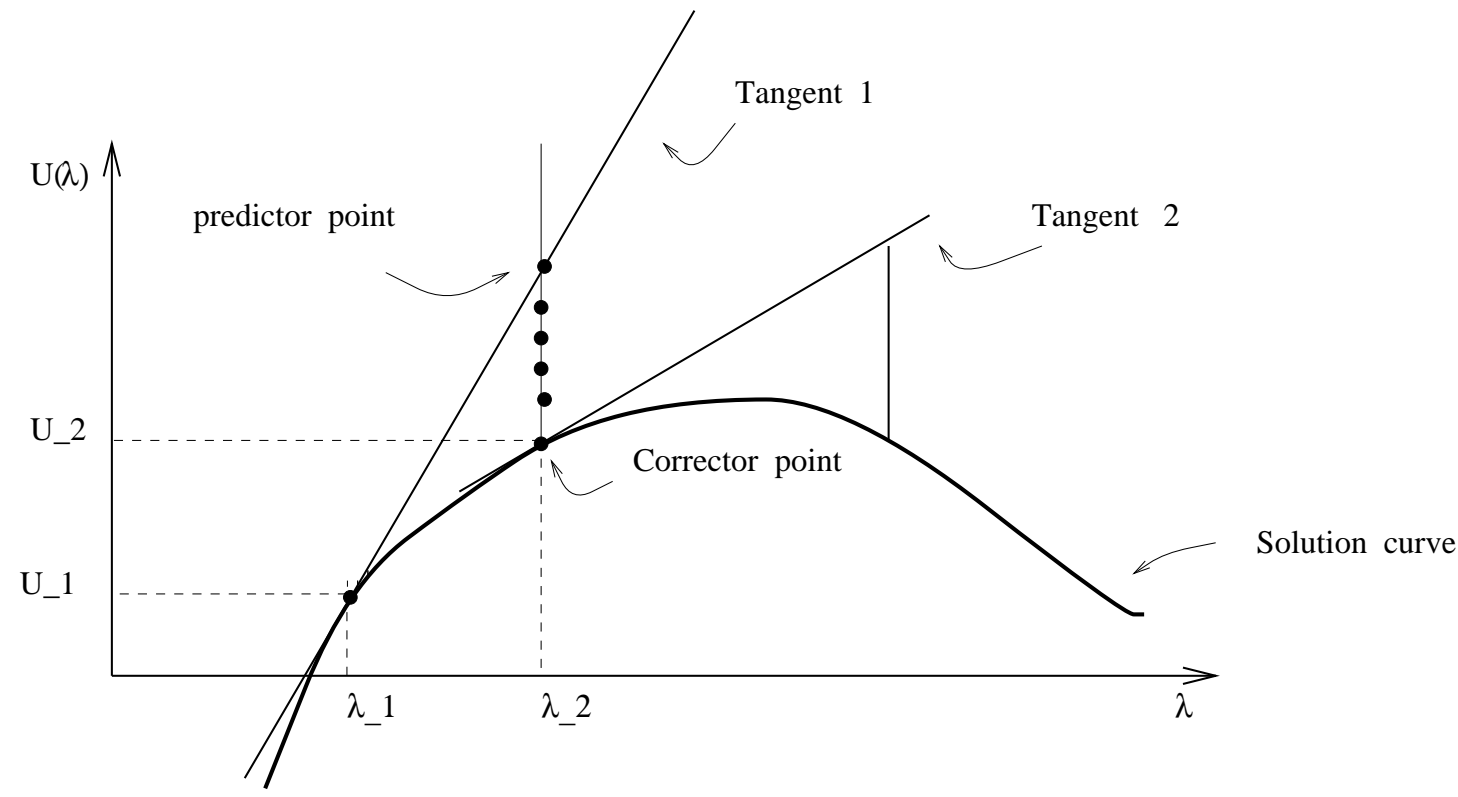

Figure 3: The classical Euler-Newton continuation. In this case the parameter $\lambda$ is suited as a parametrization for the curve. Instead of computing directly the solution for a given value of $\lambda$, we compute the solution for different values $\lambda_{i}$ distributed between the initial and the final loading. In this algorithm, the distance to the solution curve is not minimized. 


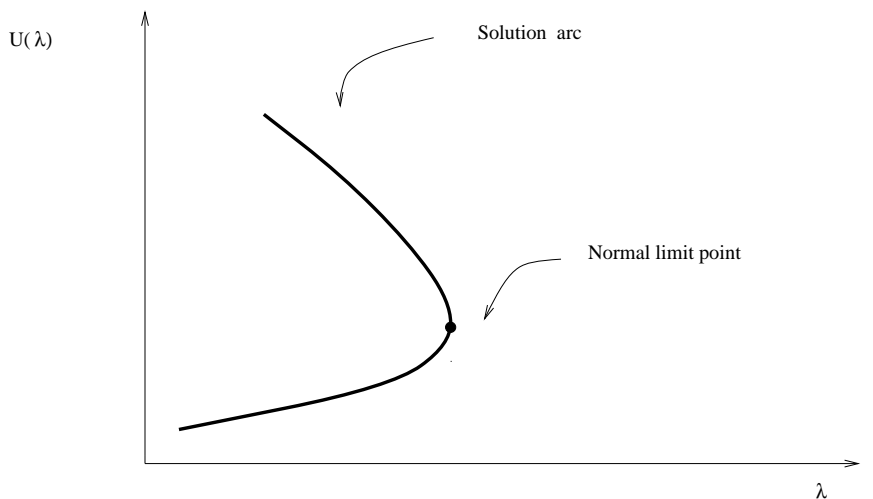

Figure 4: Normal limit point. In this case, a classical Newton-Raphson's continuation type algorithms may generate a diverging sequence. Near this point, the NewtonRaphson's algorithm can't be quadratic.
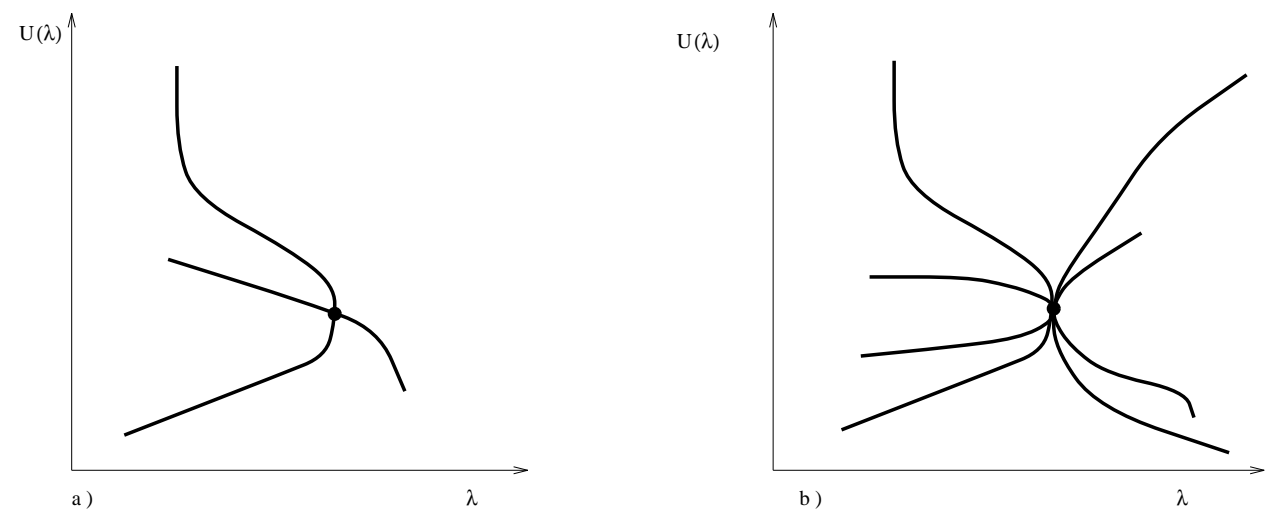

Figure 5: Bifurcation points. a) Bifurcation at turning point. b) Bifurcation at limit point. In this situations, a classical Euler-Newton's type algorithms can't converge. The arclength continuation algorithm is necessary.

$\mathrm{RR} \mathrm{n}^{\circ} 2546$ 
$\mathrm{U}(\lambda)$

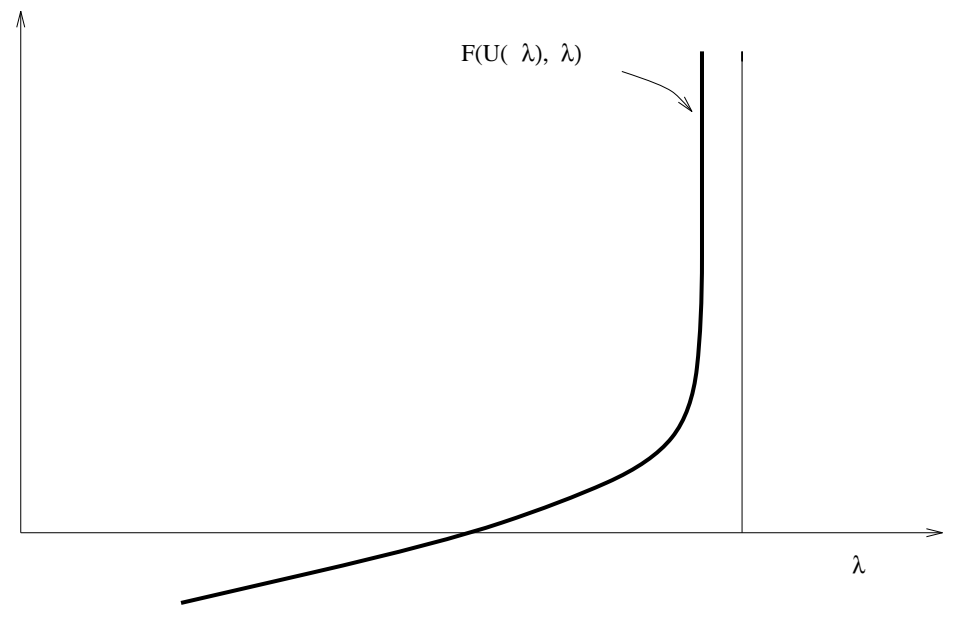

Figure 6: Strong nonlinearity. In this situation, the classical Euler-Newton's type algorithms can fail. The arclength continuation algorithm may be necessary.

\subsection{Parametrization and Arc-length continuation methods}

The sub-system (20) implicitely defines a curve of solution points. Under the following assumptions, we will prove that this curve can be parametrized with respect to the arclength parameter s.

Assumption $1: H_{1}: W_{h} \times V_{0 h} \times \mathbb{R} \rightarrow \mathbb{R}^{N E+N N}$ is a smooth map.

Assumption 2 : There is a $\operatorname{point}\left(u^{0}, \lambda^{0}\right) \in W_{h} \times V_{0 h} \times \mathbb{R}$ such that :

(1) $H_{1}\left(u^{0}, \lambda^{0}\right)=0$,

(2) the Jacobian matrix $H_{1}^{\prime}\left(u^{0}, \lambda^{0}\right)$ has a maximal rank i.e.

$$
\operatorname{rank}\left(H_{1}^{\prime}\left(u^{0}, \lambda^{0}\right)\right)=\operatorname{dim}\left(W_{h} \times V_{0 h}\right)=N E+N N .
$$

Given assumptions (32) and (33), we can choose an index i, $1 \leq i \leq N E+N N+1$, such that the submatrix of the Jacobian $H_{1}^{\prime}\left(u^{0}, \lambda^{0}\right)$ obtained by deleting the $i^{\text {th }}$ column is non-singular. It follows from the Implicit Funtion Theorem that the solution 


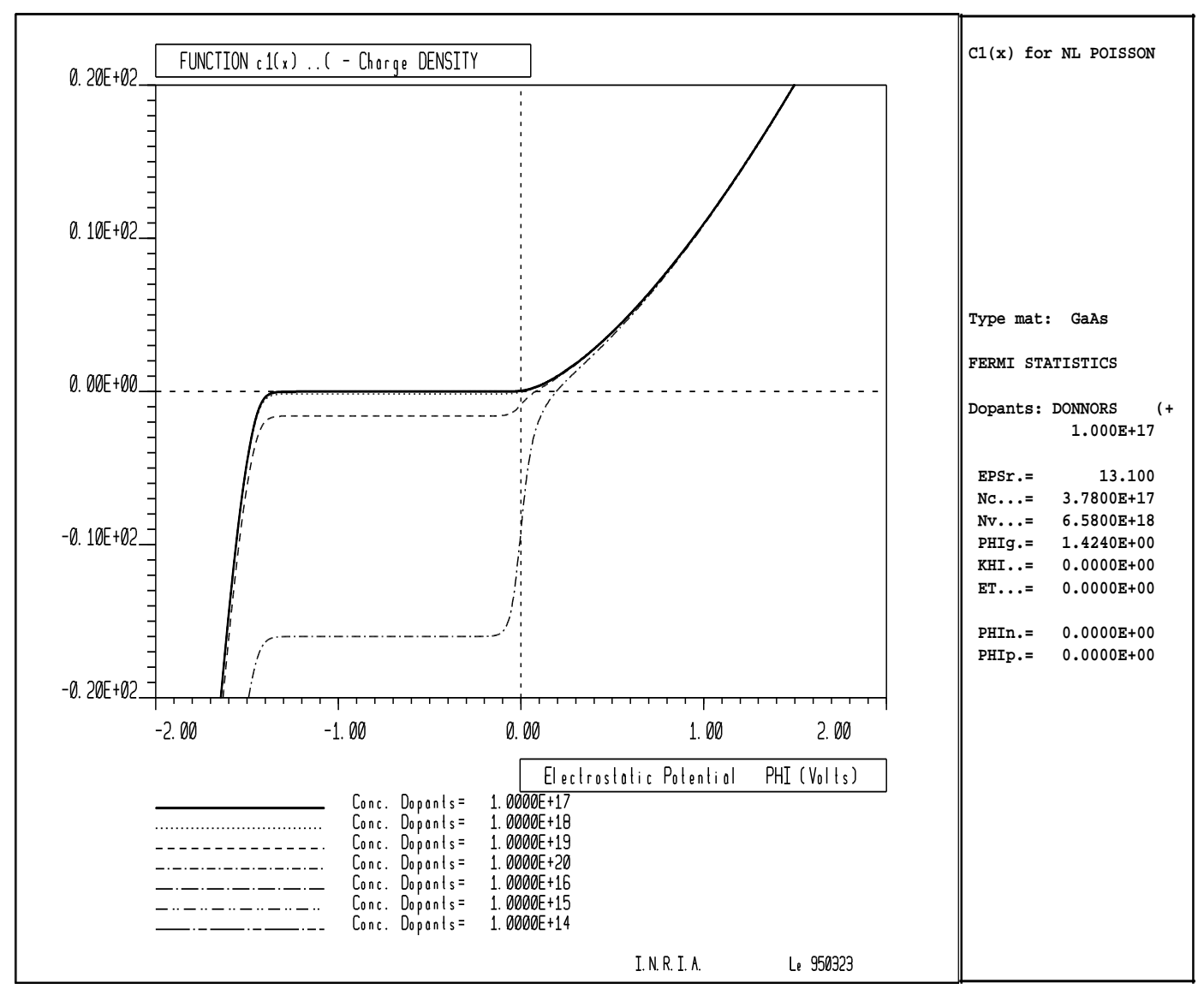

Figure 7: Variations of the $\varepsilon_{0} c_{1}$ function with respect to the electrostatic potential $\phi$ and for different doping values.

set $H_{1}^{-1}(0,0)$ can be locally parametrized with respect to the $i^{\text {th }}$ co-ordinate. By a re-parametrization, we obtain the following

Lemma 3.1 Under the assumptions (32) and (33), there exists a smooth curve $\beta \in J \rightarrow C_{1}(\beta)=(u(\beta), \lambda(\beta)) \in W_{h} \times V_{0 h} \times \mathbb{R}$ for some open interval $J$ containing zero such that for all $\beta \in J$ :

$$
\begin{gathered}
C_{1}(0)=\left(u^{0}, \lambda^{0}\right), \\
H_{1}\left(C_{1}(\beta)\right)=0,
\end{gathered}
$$




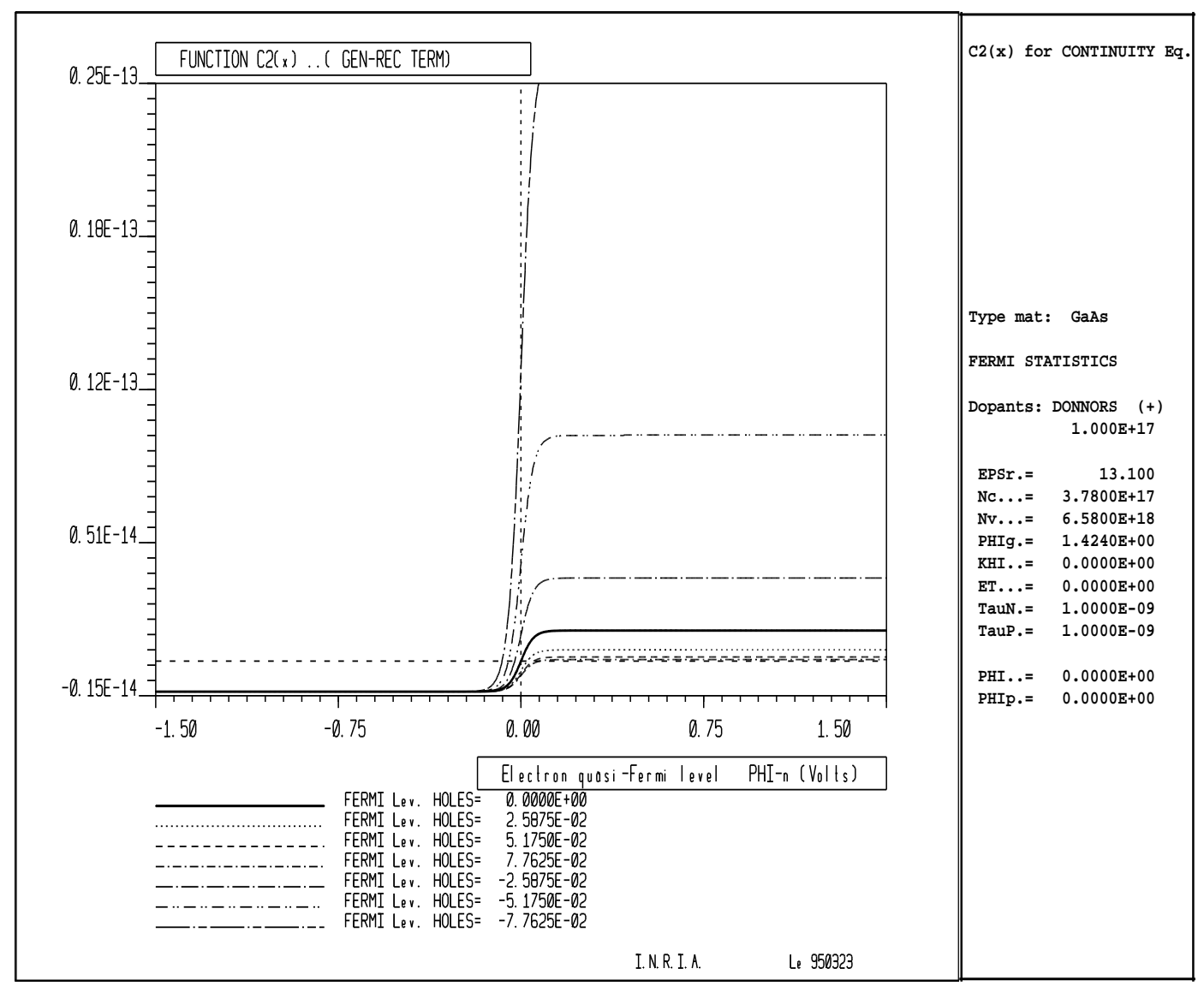

Figure 8: Variations of the $c_{2}$ (Generation-Recombinaison term)function with respect to the electron quasi-Fermi level $\phi_{n}$ and for different values of the hole quasi-Fermi level $\phi_{p}$. Electrostatic potential value is kept to 0 .

$$
\begin{gathered}
\operatorname{rank}\left(H_{1}^{\prime}\left(C_{1}(\beta)\right)=N E+N N,\right. \\
C_{1}^{\prime}(\beta) \neq 0 .
\end{gathered}
$$

It is easy to prove that the hypothesis (32) is verified. Likewise, the following results prove that the assumptions (33) are satisfied. 
Theorem 3.1 Under suitable regularity assumptions, for $g_{1} \in H^{\frac{1}{2}}(\partial \Omega)$ there exits $\left(u^{0}, \lambda^{0}\right) \in W_{h} \times V_{0 h} \times \mathbb{R}$ such that the conditions 1) and 2) in (33) are verified.

Proof : see appendix.

We have

$$
H_{1}\left(C_{1}(\beta)\right)=H_{1}(u(\beta), \lambda(\beta)) .
$$

By differentiating the equation (38) it follows that the tangent $C_{1}^{\prime}(\beta)$ satisfies the equation

$$
H_{1}^{\prime}\left(C_{1}(\beta)\right) C_{1}^{\prime}(\beta)=0
$$

and hence the tangent $C_{1}^{\prime}(\beta)$ is orthogonal to all rows of $H_{1}^{\prime}\left(C_{1}(\beta)\right)$.

There still remains a freedom of choice in parametrizing the curve. For numerical purposes, it is convenient to parametrize the curve with respect to the arclength parameter s such that

$$
d s=\left[\sum_{j=1}^{N E+N N+1}\left(\frac{d C_{1, j}(\beta)}{d \beta}\right)^{2}\right] d \beta,
$$

where $C_{1, j}$ denotes the $j^{\text {th }}$ co-ordinate of $C_{1}$.

If we replace $\beta$ by s we obtain

$$
\left\|\dot{\mathrm{C}}_{1}\right\|_{0}=1, s \in J
$$

for some new interval J. Here we have adopted the convention

$$
\dot{\mathrm{C}}_{1}=\frac{d C_{1}}{d s} .
$$

We notice that

$$
\left\|\dot{C}_{1}\right\|_{0}=1 \text {. }
$$

If we come back to the basic notations, we have

$$
N_{1}=\left\|\dot{\phi}^{k+1}(s)\right\|_{0}^{2}+\left\|\dot{D}^{k+1}(s)\right\|_{0}^{2}+\left\|\dot{\lambda}_{1}^{k+1}(s)\right\|_{0}^{2}=1 .
$$

The use of $s$ as control parameter might be too difficult in practice. Therefore, we 
can define an approximate arc length parametrization of the solution curve by replacing (20)-(41) by :

$$
\left\{\begin{array}{l}
H_{1}(u, \lambda)=0 \\
N^{1}(u, \lambda, s)=0
\end{array}\right.
$$

Here $N^{1}: W_{h} \times V_{0 h} \times \mathbb{R}^{2} \rightarrow \mathbb{R}$ is given. We shall show several choices for $N^{1}$ which makes s an approximation to "arclength" on the solution arc [33].

Then, as we shall see, thanks to the "arclength" parametrization, we can circumvent the unstabilities of the classical Newton-Raphson's method while keeping large steps in s. In order to clarify some of these adavantages, we reformulate the problem (42) as follows. We introduce $U \in W_{h} \times V_{0 h} \times \mathbb{R}$ and

$$
P_{1}: W_{h} \times V_{0 h} \times \mathbb{R}^{2} \rightarrow \mathbb{R}^{N E+N N+1}
$$

as

$$
\begin{gathered}
U \equiv(u, \lambda) \\
P_{1}(U, s) \equiv\left(\begin{array}{c}
H_{1}(u, \lambda) \\
N^{1}(u, \lambda, s)
\end{array}\right) .
\end{gathered}
$$

A solution arc of $(20)$ or $(42)$ is $U(s)=(u(s), \lambda(s))$ and it satisfies

$$
P(U(s), s)=0 \text {. }
$$

For fixed s a solution $\mathrm{U}(\mathrm{s})$ is isolated if

$$
\mathcal{A}(s)=P_{U}^{\prime}(U(s), s)=\left(\begin{array}{cc}
H_{1, u}(u(s), \lambda(s)) & H_{1, \lambda}(u(s), \lambda(s)) \\
N_{u}^{1}(u(s), \lambda(s), s) & N_{\lambda}^{1}(u(s), \lambda(s), s)
\end{array}\right)
$$

is nonsingular. Furthermore on a smooth arc $\dot{\mathrm{U}} \equiv \frac{d U}{d s}$ satisfies

$$
\mathcal{A}(s) \dot{U}(s)=\left(\begin{array}{c}
0 \\
N_{s}^{1}(u(s), \lambda(s), s)
\end{array}\right) .
$$

Now the continuation in s could proceed in exact analogy with our prior continuation in $\lambda$. However, the possible advantages of the new parametrization are clarified 
by the fact that $P_{U}$ can be nonsingular while $H_{1, u}$ is singular. Finally, with the arclength parametrization, the problem to solve is as follows :

Find $\left(u_{1}, \lambda_{1} ; u_{2}, \lambda_{2} ; u_{3}, \lambda_{3}\right): \mathbb{R} \longrightarrow\left\{W_{h} \times V_{0 h} \times \mathbb{R}\right\}^{3}$ such that :

$$
\begin{aligned}
& \left\{\begin{array}{l}
H_{1}\left(u_{1}, \lambda_{1}\right)=0, \\
N^{1}\left(u_{1}, \lambda_{1}, s\right)=0,
\end{array}\right. \\
& \left\{\begin{array}{l}
H_{2}\left(u_{2}, \lambda_{2}\right)=0, \\
N^{2}\left(u_{2}, \lambda_{2}, s\right)=0,
\end{array}\right. \\
& \left\{\begin{array}{l}
H_{3}\left(u_{3}, \lambda_{3}\right)=0, \\
N^{3}\left(u_{3}, \lambda_{3}, s\right)=0,
\end{array}\right.
\end{aligned}
$$

where

$$
u_{1}=\left(\phi^{k+1}, D^{k+1}\right), \quad u_{2,3}=\left(\phi_{n, p}^{k+1}, j_{n, p}^{k+1}\right), \quad \lambda_{1,2,3}=\lambda_{1,2,3}^{k+1} .
$$

\subsection{Choices of normalisations}

To use $s$ as the arclength parameter, we need to consider the following constraints :

$$
N_{1 i}\left(u_{i}, \lambda_{i}, s\right)=\omega_{i}\left\|\dot{u}_{i}\right\|_{0}^{2}+\left|\dot{\lambda}_{i}\right|^{2}-1=0 \quad i=1,2,3,
$$

where $\omega_{i=1,2,3}$ are introduced as normalized parameters. These will be explicitly defined in the next section.

This kind of constraints will be used to compute the velocities $\left(\dot{u}_{i}, \dot{\lambda}_{i}\right)_{i=1,2,3}$. It is not the most practical one to use in computing the solution. To this end, we will use approximations of (51).

Assuming a solution of each sub-systems (48), (49), and (50) is known, say

$$
\left[u_{i}, \lambda_{i}\right]_{i=1,2,3}=\left[u_{i}^{0}, \lambda_{i}^{0}\right]_{i=1,2,3},
$$

we set

$$
\left[u_{i}^{0}, \lambda_{i}^{0}\right]_{i=1,2,3}=\left[u_{i}\left(s_{0}\right), \lambda_{i}\left(s_{0}\right)\right]_{i=1,2,3},
$$

$\mathrm{RR} \quad \mathrm{n}^{\circ} 2546$ 
and we define over $s_{0} \leq s<s_{1}$ the following constraints for all $i=1,2,3$ :

$$
N_{2 i}\left(u_{i}, \lambda_{i}, s\right)=\omega_{i}\left\|u_{i}(s)-u_{i}\left(s_{0}\right)\right\|_{0}^{2}+\left|\lambda_{i}(s)-\lambda_{i}\left(s_{0}\right)\right|^{2}-\left(s-s_{0}\right)^{2}=0
$$

If in addition to $\left[u_{i}^{0}, \lambda_{i}^{0}\right]_{i=1,2,3}$, we know the velocities $\left(\dot{u}_{i}, \dot{\lambda}_{i}\right)_{i=1,2,3}$, then we can use the following constraints for all $i=1,2,3$ :

$$
N_{3 i}\left(u_{i}, \lambda_{i}, s\right)=\omega_{i} \dot{u}_{i}^{*}\left(s_{0}\right)\left[u_{i}(s)-u_{i}\left(s_{0}\right)\right]+\dot{\lambda}_{i}\left(s_{0}\right)\left[\lambda_{i}(s)-\lambda_{i}\left(s_{0}\right)\right]-\left(s-s_{0}\right)=0 .
$$

The vector $\dot{u}_{i}^{*}$ is a row vector and corresponds to the dual element to $\dot{u}_{i}$ such that

$$
\dot{u}_{i}^{*}\left(s_{0}\right) \dot{u}_{i}\left(s_{0}\right)=\left\|\dot{u}_{i}\left(s_{0}\right)\right\|_{0}^{2} .
$$

The existence of such an element is given by the Hahnn-Banach theorem [46]. The constraints (52) and (53) are called the pseudo-arclength normalizations [33]. For our theoretical and numerical analysis, we will use the constraints (53).

\subsection{Arclength continuation about regular and limit points}

At first, we will justify the arclength continuation procedure, applied to our problem, using the normalization $\left(N_{3 i}\right)_{i=1,2,3}$ on solution arcs composed of "regular points". Let $\left[u_{i}^{0}, \lambda_{i}^{0}\right]_{i=1,2,3}$ be a solution of sub-systems (48), (49), and (50) respectively and let $\left(\dot{u}_{i}, \dot{\lambda}_{i}\right)_{i=1,2,3}$ satisfy

$$
\begin{aligned}
& \text { a) } \quad H_{i, u_{i}}^{0} \dot{u}_{i}^{0}+H_{i, \lambda_{i}} \dot{\lambda}_{i}^{0}=0 \quad i=1,2,3, \\
& \text { b) } \quad \omega_{i}\left\|\dot{u}_{i}\right\|_{0}^{2}+\left|\dot{\lambda_{i}}\right|^{2}=1 \quad i=1,2,3 .
\end{aligned}
$$

Definition 3.1 We say that $\left[u_{i}^{0}, \lambda_{i}^{0}\right]_{i=1,2,3}$ are regular points if in addition to (54) a), b) the operators

$$
H_{i, u_{i}}^{0}=H_{i, u_{i}}\left(u_{i}^{0}, \lambda_{i}^{0}\right)_{1,2,3} \text { are nonsingular. }
$$


Theorem 3.2 Let the velocities $\left(\dot{u}_{i}, \dot{\lambda}_{i}\right)_{i=1,2,3}$ satisfy the conditions a) and $\left.b\right)$ in (54). Let $H_{i}\left(u_{i}, \lambda_{i}\right), i=1,2,3$ have two continuous derivatives in some ball about $\left[u_{i}^{0}, \lambda_{i}^{0}\right]_{i=1,2,3}$. Then each of the sub-systems (48), (49) and (50) has a unique smooth arc of solution $\left[u_{i}(s), \lambda_{i}(s)\right]_{i=1,2,3}$ on $\left|s-s_{0}\right| \leq \rho$ for some sufficiently small $\rho>0$.

\section{Proof:}

We will prove that the Frechet derivatives $\mathcal{A}_{i=1,2,3}\left(s_{0}\right)$ are nonsingular. We will study only the operator $\mathcal{A}_{2}\left(s_{0}\right)$. The analysis of the other operators is similar. We recall that the operator $\mathcal{A}_{2}\left(s_{0}\right)$ is given by

$$
\mathcal{A}_{2}\left(s_{0}\right)=\left(\begin{array}{cc}
H_{2, u_{2}}\left(u\left(s_{0}\right), \lambda_{2}\left(s_{0}\right)\right) & H_{2, \lambda_{2}}\left(u\left(s_{0}\right), \lambda_{2}\left(s_{0}\right)\right) \\
\omega_{2} \dot{u}_{2}^{*}\left(s_{0}\right) & \dot{\lambda}_{2}\left(s_{0}\right)
\end{array}\right) .
$$

We can first prove (see appendix) that under suitable assumptions and for $g_{2} \in$ $H^{\frac{1}{2}}(\partial \Omega)$ and for $\mathrm{h}$ sufficiently small, the operator $H_{2, u_{2}}^{0}$ is nonsingular.

Now if $\dot{\lambda}_{2}=0$ then the conditions a) in (54) and the proposition (.1) (see appendix) imply $\dot{u}_{2}=0$. This contradicts b) in (54) so $\dot{\lambda}_{2} \neq 0$. By Gauss elimination, the operator $\mathcal{A}_{2}\left(s_{0}\right)$ is nonsingular iff :

$$
\dot{\lambda}_{2}-\omega_{2} \dot{u}_{2}^{*}\left(H_{2, u_{2}}^{0}\right)^{-1} H_{2, \lambda_{2}}^{0}
$$

is nonsingular. By using the conditions a) in (54), we have

$$
\omega_{2} \frac{\dot{u}_{2}^{*}}{\dot{\lambda}_{2}}=-\left(H_{2, u_{2}}^{0}\right)^{-1} H_{2, \lambda_{2}}^{0}
$$

So, using the above, the condition (57) is equivalent to the following

$$
\dot{\lambda}_{2}+\omega_{2} \frac{\dot{u}_{2}^{*} \dot{u}_{2}}{\dot{\lambda}_{2}} \neq 0
$$

which is true by construction of the dual vector $\dot{u}_{2}^{*}$. We finally deduce that the operator $\mathcal{A}_{2}\left(s_{0}\right)$ is nonsingular. The results of theorem 3.2 now follows from the application of Implicit Function Theorem [48] at $\left[u_{i}, \lambda_{i}, s\right]=\left[u_{i}^{0}, \lambda_{i}^{0}, s_{0}\right], i=1,2,3$. 
Definition 3.2 We call $\left[u_{i}, \lambda_{i}\right]_{i=1,2,3}$ normal limit points if the conditions a) and b) in (54) hold, but in place of (55) we have:

$$
\begin{aligned}
& \text { a) } \quad \operatorname{dim}\left(\mathcal{N}\left(H_{i, u_{i}}^{0}\right)\right)=\operatorname{codim}\left(\mathcal{R}\left(H_{i, u_{i}}^{0}\right)\right)=1 i=1,2,3, \\
& \text { b) } \quad H_{i, \lambda_{i}}^{0} \notin \mathcal{R}\left(H_{i, u_{i}}^{0}\right) \quad i=1,2,3 .
\end{aligned}
$$

Limit points are illustrated by figures 4,5 .

Assuming that we have to solve some general parametric system : Find $u \in V$ such that

$$
H(u(\lambda), \lambda)=0,
$$

where $\lambda$ is a parameter and $\mathrm{V}$ is some Banach space. Assuming that the arc of solutions $(u(\lambda), \lambda)$ of the problem $(60)$ is composed of normal limit points. We can also justify the arclength continuation procedure using the normalization $N_{3}$.

Proposition 3.1 Let $\left[u^{0}, \lambda^{0}\right]$ be a normal limit solution of the problem (60). Let $H(u, \lambda)$ have two continuous derivatives in some ball about $\left[u^{0}, \lambda^{0}\right]$. Then the system (60) has a unique smooth arc of solution $[u(s), \lambda(s)]$ using constraint $N_{3}$ on $\left|s-s_{0}\right| \leq \rho$ for some sufficiently small $\rho>0$.

Remarks 3.1 1) The condition b) in (59) implies $\dot{\lambda}_{i}=0 \quad i=1,2,3$.

2) The use of the arclength continuation can also be used for an automatic adaptation of the boundary data $g_{1}$ and $g_{2}$ (applied potential) [51]. For some semiconductor devices and for some values of the applied potential, we can have multiple solutions. For the Tyristor, for example, we have multiple solutions (or multiple currents I) for some applied potential. In this case, the curve solution $I=I\left(g_{2}\right)$ encountered two normal limit points see figure 9. As mentionned, the solution can be computed, in the case of limit points, by using arclength continuation procedures.

In our case, we will only be interested by regular points, but algorithms presented here, are general as they can include limit and bifurcation points. For the sake of the simplicity, the index star $(*)$ will be omitted. 


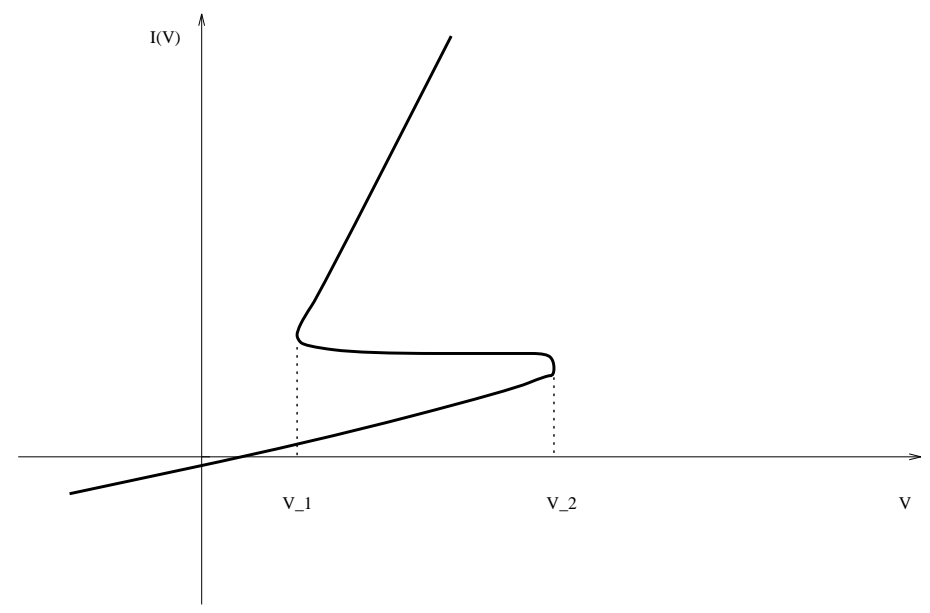

Figure 9: The values $v_{1}$, and $v_{2}$ of the applied potential correspond to two limit points. Besides, if $v_{1} \leq v \leq v_{2}$ multiple values may be obtained. In this case, the arclength continuation method is necessary.

\section{Detailed arclength continuation type algorithms}

\subsection{Algorithm 2:}

Step 1 : a) Initialization of the continuation :

For $\lambda_{1,2,3}^{0}=0, s_{1,2,3}^{0}=0, \quad\left(\phi^{0}, D^{0}, \phi_{n, p}^{0}, j_{n, p}^{0}\right)$ given, we choose $\lambda_{1,2,3}^{1}>\lambda_{1,2,3}^{0}$ and set $\Delta \lambda_{1,2,3}=\lambda_{1,2,3}-\lambda_{1,2,3}^{0}$. We then solve the following decoupled nonlinear systems, by the classical Newton-Raphson's algorithm to compute $\left(\phi^{1}, D^{1}, \phi_{n}^{1}, j_{n}^{1}, \phi_{p}^{1}, \phi_{p}^{1}\right)$ :

$H_{1}\left(\phi^{1}, D^{1}, \lambda_{1}^{1}, \phi_{n}^{0}, \phi_{p}^{0}\right)=0, \quad$ initial point for Poisson's equation,

$$
H_{2}\left(\phi_{n}^{1}, j_{n}^{1}, \lambda_{2}^{1}, \phi^{1}, \phi_{p}^{0}\right)=0, \quad \text { initial point for electron transport equations }
$$

$$
H_{3}\left(\phi_{p}^{1}, j_{p}^{1}, \lambda_{3}^{1}, \phi^{1}, \phi_{n}^{1}\right)=0, \quad \text { initial point for hole transport equations. }
$$


b) Computation of the predictor-corrector continuation parameters :

$$
\begin{aligned}
& \omega_{l_{1}}=\frac{\left|\Delta \lambda_{1}\right|}{\|\Delta \phi\|_{l^{2}}}, \quad \omega_{l_{2}}=\frac{\left|\Delta \lambda_{2}\right|}{\left\|\Delta \phi_{n}\right\|_{l^{2}}} \quad \omega_{l_{3}}=\frac{\left|\Delta \lambda_{3}\right|}{\left\|\Delta \phi_{p}\right\|_{l^{2}}}, \\
& \omega_{p_{d}}=\frac{\left|\Delta \lambda_{1}\right|}{\|\Delta D\|_{l^{2}}}, \quad \omega_{p_{n}}=\frac{\left|\Delta \lambda_{2}\right|}{\left\|\Delta j_{n}\right\|_{l^{2}}}, \quad \omega_{p_{p}}=\frac{\left|\Delta \lambda_{3}\right|}{\left\|\Delta j_{p}\right\|_{l^{2}}},
\end{aligned}
$$

$\Delta s_{1}=\sqrt{3}\left|\Delta \lambda_{1}\right|, \quad$ initial steplength for Poisson's equation,

$\Delta s_{2}=\sqrt{3}\left|\Delta \lambda_{2}\right|$ initial steplength for electron transport equations,

$\Delta s_{2}=\sqrt{3}\left|\Delta \lambda_{3}\right|, \quad$ initial steplength for hole transport equations.

\section{Step 2 : Continuation loop}

\section{a) Computation of the velocities}

To compute the velocities associated with the above sub-systems, we solve respectively, for all $k \geq 1$, the following linear sub-systems :

$$
\left\{\begin{array}{l}
\frac{\partial H_{1}\left(\phi^{k}, D^{k}, \lambda_{1}^{k}, \phi_{n}^{k}, \phi_{p}^{k}\right)}{\partial \phi} \dot{\phi}^{k}+\frac{\partial H_{1}\left(\phi^{k}, D^{k}, \lambda_{1}^{k}, \phi_{n}^{k}, \phi_{p}^{k}\right)}{\partial D} \dot{D}^{k}+ \\
\frac{\partial H_{1}\left(\phi^{k}, D^{k}, \lambda_{1}^{k}, \phi_{n}^{k}, \phi_{p}^{k}\right)}{\partial \lambda_{1}} \dot{\lambda}_{1}^{k}=0 \\
\omega_{l_{1}}\left\|\dot{\phi}^{k}\right\|_{l^{2}}+\omega_{p_{d}}\left\|\dot{D}^{k}\right\|_{l^{2}}^{2}+\left|\dot{\lambda}_{1}^{k}\right|^{2}=1
\end{array}\right.
$$




$$
\begin{aligned}
& \left\{\begin{array}{l}
\frac{\partial H_{2}\left(\phi_{n}^{k}, j_{n}^{k}, \lambda_{2}^{k}, \phi^{k}, \phi_{p}^{k}\right)}{\partial \phi_{n}} \dot{\phi}_{n}^{k}+\frac{\partial H_{2}\left(\phi_{n}^{k}, j_{n}^{k}, \lambda_{2}^{k}, \phi_{n}^{k}, \phi_{p}^{k}\right)}{\partial j_{n}} \dot{j}_{n}^{k}+ \\
\frac{\partial H_{2}\left(\phi_{n}^{k}, j_{n}^{k}, \lambda_{2}^{k}, \phi^{k}, \phi_{p}^{k}\right)}{\partial \lambda_{2}} \dot{\lambda}_{2}^{k}=0, \\
\omega_{l_{2}}\left\|\dot{\phi}_{n}^{k}\right\|_{l^{2}}+\omega_{p_{n}}\left\|\dot{j}_{n}^{k}\right\|_{l^{2}}^{2}+\left|\dot{\lambda}_{2}^{k}\right|^{2}=1,
\end{array}\right. \\
& \left\{\begin{array}{l}
\frac{\partial H_{3}\left(\phi_{p}^{k}, j_{p}^{k}, \lambda_{2}^{k}, \phi^{k}, \phi_{n}^{k}\right)}{\partial \phi_{n}} \dot{\phi}_{p}^{k}+\frac{\partial H_{3}\left(\phi_{p}^{k}, j_{p}^{k}, \lambda_{3}^{k}, \phi^{k}, \phi_{n}^{k}\right)}{\partial j_{p}} \dot{j}_{p}^{k}+ \\
\frac{\partial H_{3}\left(\phi_{p}^{k}, j_{p}^{k}, \lambda_{3}^{k}, \phi^{k}, \phi_{n}^{k}\right)}{\partial \lambda_{2}} \dot{\lambda}_{3}^{k}=0, \\
\omega_{l_{3}}\left\|\dot{\phi}_{p}^{k}\right\|_{l^{2}}+\omega_{p_{p}}\left\|\dot{j}_{p}^{k}\right\|_{l^{2}}^{2}+\left|\dot{\lambda}_{3}^{k}\right|^{2}=1,
\end{array}\right.
\end{aligned}
$$

\section{b) Euler's predictor step :}

$$
\begin{gathered}
\left\{\begin{array}{l}
\phi^{k+1,0}=\phi^{k}+\Delta s_{1} \dot{\phi}^{k}, \\
D^{k+1,0}=D^{k}+\Delta s_{1} \dot{D}^{k} \\
\lambda_{1}^{k+1,0}=\lambda_{1}^{k}+\Delta s_{1} \dot{\lambda}_{1}^{k},
\end{array}\right. \\
\left\{\begin{array}{l}
\phi_{n}^{k+1,0}=\phi_{n}^{k}+\Delta s_{2} \dot{\phi}_{n}^{k}, \\
j_{n}^{k+1,0}=j_{n}^{k}+\Delta s_{2} \mathrm{j}_{n}^{k}, \\
\lambda_{2}^{k+1,0}=\lambda_{2}^{k}+\Delta s_{2} \dot{\lambda}_{2}^{k},
\end{array}\right. \\
\left\{\begin{array}{l}
\phi_{p}^{k+1,0}=\phi_{p}^{k}+\Delta s_{3} \dot{\phi}_{p}^{k} \\
j_{p}^{k+1,0}=j_{p}^{k}+\Delta s_{3} \mathrm{j}_{p}^{k} \\
\lambda_{3}^{k+1,0}=\lambda_{3}^{k}+\Delta s_{3} \dot{\lambda}_{3}^{k} .
\end{array}\right.
\end{gathered}
$$




\section{c)Newton-Raphson's corrector step :}

To compute the solution $\left(\phi^{k+1}, D^{k+1}, \lambda_{1}^{k+1}, \phi_{n}^{k+1}, j_{n}^{k+1}, \lambda_{2}^{k+1}, \phi_{n}^{k+1}, j_{n}^{k+1}, \lambda_{3}^{k+1}\right)$, we solve, respectively, by the classical Newton-Raphson's algorithm, the following nonlinear systems :

$$
\begin{aligned}
& \left\{\begin{array}{l}
H_{1}\left(\phi^{k+1}, D^{k+1}, \lambda_{1}^{k+1}, \phi_{n}^{k}, \phi_{p}^{k}\right)=0, \\
N_{31}\left(\phi^{k+1}, D^{k+1}, \lambda_{1}^{k+1}, s_{1}^{k+1}\right)=0,
\end{array}\right. \\
& \left\{\begin{array}{l}
H_{2}\left(\phi_{n}^{k+1}, j_{n}^{k+1}, \lambda_{2}^{k+1}, \phi^{k+1}, \phi_{p}^{k}\right)=0, \\
N_{32}\left(\phi_{n}^{k+1}, j_{n}^{k+1}, \lambda_{2}^{k+1}, s_{2}^{k+1}\right)=0,
\end{array}\right. \\
& \left\{\begin{array}{l}
H_{3}\left(\phi_{p}^{k+1}, j_{p}^{k+1}, \lambda_{3}^{k+1}, \phi^{k+1}, \phi_{n}^{k+1}\right)=0, \\
N_{33}\left(\phi_{p}^{k+1}, j_{p}^{k+1}, \lambda_{3}^{k+1}, s_{3}^{k+1}\right)=0,
\end{array}\right.
\end{aligned}
$$

where $s_{i}^{k+1}=s_{i}^{k}+\Delta s_{i} \quad i=1,2,3$.

\section{d) Steplength adaptation :}

We choose new steplengths for each sub-systems $\Delta s_{1}, \Delta s_{2}$, and $\Delta s_{3}$.

\section{Step 3 : Convergence test}

Setting

$$
\begin{aligned}
& e_{1}=\max \left(\frac{\left\|\phi^{k+1}-\phi^{k}\right\|}{\left\|\phi^{k+1}\right\|}, \frac{\left\|D^{k+1}-D^{k}\right\|}{\left\|D^{k+1}\right\|}\right), \\
& e_{2}=\max \left(\frac{\left\|\phi_{n}^{k+1}-\phi_{n}^{k}\right\|}{\left\|\phi_{n}^{k+1}\right\|}, \frac{\left\|j_{n}^{k+1}-j_{n}^{k}\right\|}{\left\|j_{n}^{k+1}\right\|}\right), \\
& e_{3}=\max \left(\frac{\left\|\phi_{p}^{k+1}-\phi_{p}^{k}\right\|}{\left\|\phi_{p}^{k+1}\right\|}, \frac{\left\|j_{p}^{k+1}-j_{p}^{k}\right\|}{\left\|j_{p}^{k+1}\right\|}\right) .
\end{aligned}
$$


The predictor-corrector process is stopped when

$$
\max \left(e_{1}, e_{2}, e_{3}\right) \leq \varepsilon_{2},
$$

where $\varepsilon_{2}$ is sufficiently small.

\section{$\underline{\text { END ALGORITHM } 2}$}

The values of $\varepsilon_{2}$ depend of the values of $\varepsilon_{1}$ chosen to stop the Newton-Raphson's algorithm in the corrector step. Hence, they are computer precision dependent.

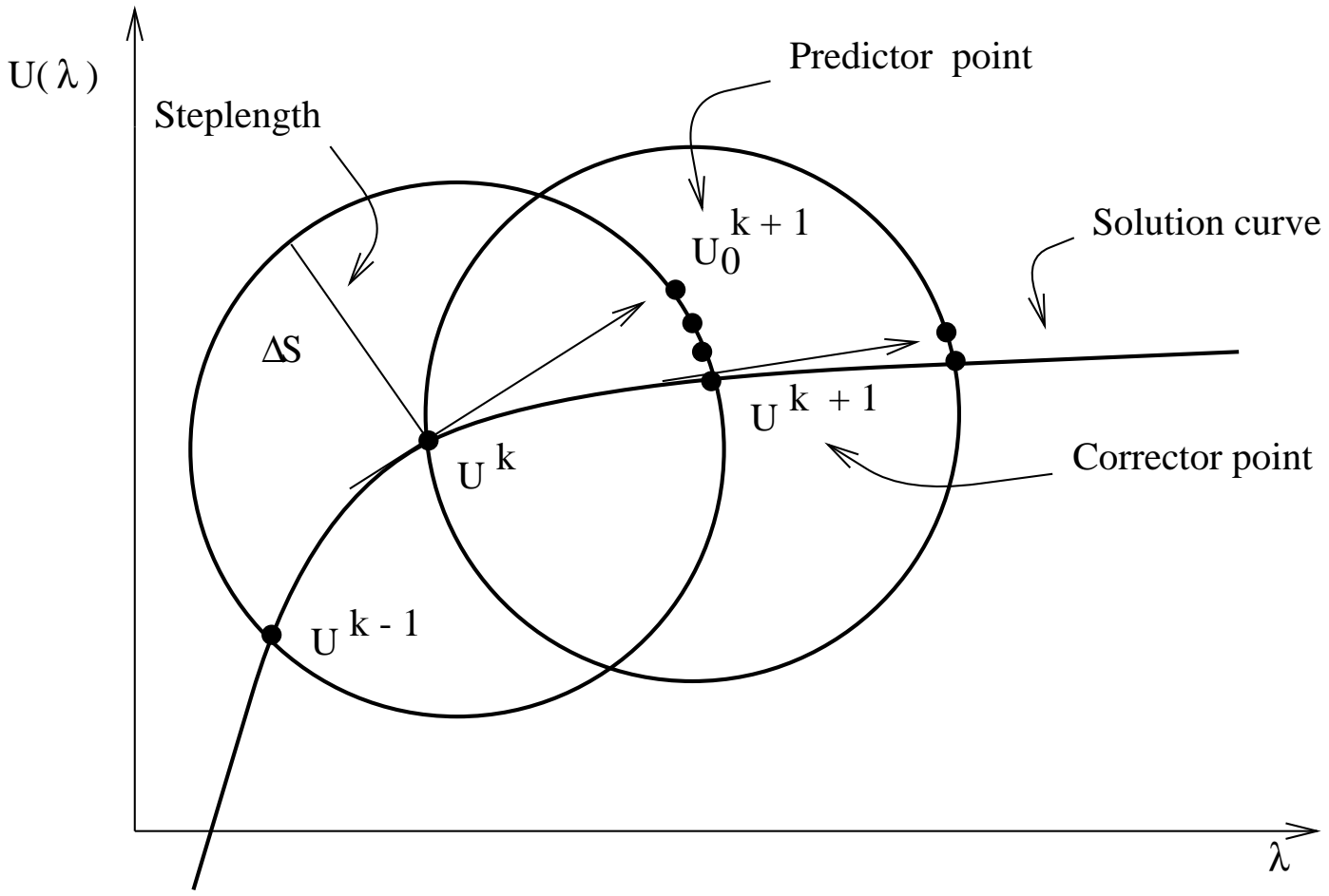

Figure 10: Algorithm 2: Arclength continuation type algorithm with constraint $N_{2}$. The suited path is not orthogonal to the velocity vector. 
Remark 4.1 The convergence of the algorithm 2 is not sensitive to the norm $\|$. chosen in the convergence test. Different norms have been tested.

$$
\|\cdot\|_{0}, \quad\|\cdot\|_{l^{1}}, \quad\|\cdot\|_{l^{2}}, \quad\|\cdot\|_{\infty} \cdot \square
$$

\subsection{On the computation of the continuation velocities}

In this section, we want to present the strategies used to compute the velocities in the continuation algorithm described above. The basic idea is the block Gaussian elimination. We will restrict our description to the sub-system associated to the electron continuity equations. The analysis of the other sub-systems is similar. To compute the velocities we have to solve for each $k \geq 1$ the sub-system

$$
\left\{\begin{array}{l}
\frac{\partial H_{21}\left(\phi_{n}^{k}, j_{n}^{k}, \lambda_{2}^{k}, \phi^{k}, \phi_{p}^{k}\right)}{\partial \phi_{n}} \dot{\phi}_{n}^{k}+\frac{\partial H_{21}\left(\phi_{n}^{k}, j_{n}^{k}, \lambda_{2}^{k}, \phi_{n}^{k}, \phi_{p}^{k}\right)}{\partial j_{n}} \mathrm{j}_{n}^{k}+ \\
\frac{\partial H_{21}\left(\phi_{n}^{k}, j_{n}^{k}, \lambda_{2}^{k}, \phi^{k}, \phi_{p}^{k}\right)}{\partial \lambda_{2}} \dot{\lambda}_{2}^{k}=0, \\
\frac{\partial H_{22}\left(\phi_{n}^{k}, j_{n}^{k}, \lambda_{2}^{k}, \phi^{k}, \phi_{p}^{k}\right)}{\partial \phi_{n}} \dot{\phi}_{n}^{k}+\frac{\partial H_{22}\left(\phi_{n}^{k}, j_{n}^{k}, \lambda_{2}^{k}, \phi_{n}^{k}, \phi_{p}^{k}\right)}{\partial j_{n}} \mathrm{j}_{n}^{k}+ \\
\frac{\partial H_{22}\left(\phi_{n}^{k}, j_{n}^{k}, \lambda_{2}^{k}, \phi^{k}, \phi_{p}^{k}\right)}{\partial \lambda_{2}} \dot{\lambda}_{2}^{k}=0, \\
\omega_{l_{2}}\left\|\dot{\phi}_{n}^{k}\right\|_{l^{2}}+\omega_{p_{n}}\left\|\dot{j}_{n}^{k}\right\|_{l^{2}}^{2}+\left|\dot{\lambda}_{2}^{k}\right|^{2}=1 .
\end{array}\right.
$$

We set

$$
K_{2}=\left(\begin{array}{ll}
\frac{\partial H_{21}\left(\phi_{n}^{k}, j_{n}^{k}, \lambda_{2}^{k}, \phi^{k}, \phi_{p}^{k}\right)}{\partial \phi_{n}} & \frac{\partial H_{21}\left(\phi_{n}^{k}, j_{n}^{k}, \lambda_{2}^{k}, \phi^{k}, \phi_{p}^{k}\right)}{\partial j_{n}} \\
\frac{\partial H_{22}\left(\phi_{n}^{k}, j_{n}^{k}, \lambda_{2}^{k}, \phi^{k}, \phi_{p}^{k}\right)}{\partial \phi_{n}} & \frac{\partial H_{22}\left(\phi_{n}^{k}, j_{n}^{k}, \lambda_{2}^{k}, \phi^{k}, \phi_{p}^{k}\right)}{\partial j_{n}}
\end{array}\right) .
$$

The two first equations of the sub-system (76) are now equivalent to the following equation

$$
K_{2}\left(\dot{\phi}_{n}^{k}, \dot{j}_{n}^{k}\right)=-\frac{\partial H_{2}}{\partial \lambda_{2}} \dot{\lambda}_{2}^{k}
$$


This implies

$$
\left(\dot{\phi}_{n}^{k}, \dot{j}_{n}^{k}\right)=-K_{2}^{-1} \frac{\partial H_{2}}{\partial \lambda_{2}} \dot{\lambda}_{2}^{k}
$$

We now set

$$
\left(\Delta \phi_{n 1}, \Delta j_{n 1}\right)=-K_{2}^{-1} \frac{\partial H_{2}}{\partial \lambda_{2}}
$$

that is

$$
K_{2}\left(\Delta \phi_{n 1}, \Delta j_{n 1}\right)=-\frac{\partial H_{2}}{\partial \lambda_{2}}
$$

If we use the equation (80), the sub-system to solve is reduced to find $\left(\dot{\phi}_{n}, \dot{j}_{n}, \dot{\lambda}_{2}\right)$ such that:

$$
\left\{\begin{array}{l}
\left(\dot{\phi}_{n}^{k}, \dot{j}_{n}^{k}\right)=\left(\Delta \phi_{n 1}, \Delta j_{n 1}\right) \dot{\lambda}_{2}^{k}, \\
\omega_{l_{2}}\left\|\dot{\phi}_{n}^{k}\right\|_{l^{2}}^{2}+\omega_{p_{n}}\left\|\dot{j}_{n}^{k}\right\|_{l^{2}}^{2}+\left|\dot{\lambda}_{2}^{k}\right|^{2}=1 .
\end{array}\right.
$$

That is

$$
\left|\dot{\lambda}_{2}^{k}\right|^{2}=\frac{1}{\left[\omega_{l_{2}}\left\|\Delta \phi_{n 1}\right\|_{l^{2}}^{2}+\omega_{p_{n}}\left\|\Delta j_{n 1}\right\|_{l^{2}}^{2}+1\right]}
$$

To obtain the stationary state, the function $\lambda_{2}$ must be increasing with respect to s. So, we take $\dot{\lambda}_{2}>0$. Consequently, we have

$$
\dot{\lambda}_{2}^{k}=\frac{1}{\sqrt{\omega_{l_{2}}\left\|\Delta \phi_{n 1}\right\|_{l^{2}}^{2}+\omega_{p_{n}}\left\|\Delta j_{n 1}\right\|_{l^{2}}^{2}+1}} .
$$

By the first equation in (82), we obtain

$$
\begin{aligned}
& \dot{\phi}_{n}^{k}=\Delta \phi_{n 1} \dot{\lambda}_{2}^{k}, \\
& \dot{j}_{n}^{k}=\Delta j_{n 1} \dot{\lambda}_{2}^{k} .
\end{aligned}
$$

The computation of the velocities $\left(\dot{\lambda}_{2}^{k}, \dot{\phi}_{n}^{k}, \mathrm{j}_{n}^{k}\right)$ is now reduced to the computation of the quantities $\left(\Delta \phi_{n 1}, \Delta j_{n 1}\right)$. These latter are obtained by solving the linear system (81) with two equations and two unknowns. The system (81) is equivalent to the following one

$\mathrm{RR} \mathrm{n}^{\circ} 2546$ 


$$
\left\{\begin{array}{l}
\frac{\partial H_{21}\left(\phi_{n}^{k}, j_{n}^{k}, \lambda_{2}^{k}, \phi^{k}, \phi_{p}^{k}\right)}{\partial \phi_{n}} \Delta \phi_{n 1}+\frac{\partial H_{21}\left(\phi_{n}^{k}, j_{n}^{k}, \lambda_{2}^{k}, \phi_{n}^{k}, \phi_{p}^{k}\right)}{\partial j_{n}} \Delta j_{n 1}= \\
-\frac{\partial H_{21}}{\partial \lambda_{2}}, \\
\frac{\partial H_{22}\left(\phi_{n}^{k}, j_{n}^{k}, \lambda_{2}^{k}, \phi^{k}, \phi_{p}^{k}\right)}{\partial \phi_{n}} \Delta \phi_{n 1}+\frac{\partial H_{22}\left(\phi_{n}^{k}, j_{n}^{k}, \lambda_{2}^{k}, \phi_{n}^{k}, \phi_{p}^{k}\right)}{\partial j_{n}} \Delta j_{n 1}=0 .
\end{array}\right.
$$

Using the derivative of $H_{21}, H_{22}$, we have to solve

$$
\begin{gathered}
\int_{\Omega}\left(\frac{-N_{\phi_{n}}^{\prime}\left(x, \phi^{k}, \phi_{n}^{k}\right)}{q \mu_{n}(x) N\left(x, \phi^{k}, \phi_{n}^{k}\right)^{2}} j_{n}^{k} v_{2} \Delta \phi_{n 1}+\operatorname{div}\left(v_{2}\right) \Delta \phi_{n 1}\right) d x+ \\
\int_{\Omega}\left[q \mu_{n}(x) N\left(x, \phi^{k}, \phi_{n}^{k}\right)\right]^{-1} \Delta j_{n 1} v_{2} d x=0 \quad \forall v_{2} \in V_{0 h}, \\
\int_{\Omega}\left[\frac{A_{2}}{\bar{\lambda}_{2}^{k}}+c_{2, \phi_{n}}^{\prime}\left(x, \phi^{k}, \phi_{n}^{k}, \phi_{p}^{k}\right)\right] \Delta \phi_{n 1} w_{2} d x-\int_{\Omega} \operatorname{div}\left(\Delta j_{n 1}\right) w_{2} d x= \\
\int_{\Omega} \frac{A_{2}}{\left(\bar{\lambda}_{2}^{k}\right)^{2}} w_{2} d x \quad \forall w_{2} \in W_{h} .
\end{gathered}
$$

One can remark that the matrix associated with the above system has the strucure

$$
\left(\begin{array}{cc}
A & B^{t}+J \\
B & C
\end{array}\right) .
$$

We point out that this matrix is $(N E+N N) \times(N E+N N)$, and is neither symmetric nor positive-definite. Besides, in two dimensional case, its storage becomes expensive. To avoid this difficulty we use the fact that our (MFE) approximation is of lowest order. So, we can eliminate element by element the primal variable $\Delta \phi_{n 1}$ which is locally constant. To this end, we project the second equation on element $T \in \mathcal{T}_{h}$. Hence, we have

$$
\left[1+\bar{\lambda}_{2}^{k} A_{2}^{-1} c_{2, \phi_{n}}^{\prime}\left(x, \phi_{T}^{k}, \phi_{n T}^{k}, \phi_{p T}^{k}\right)\right] \Delta \phi_{n 1 T}=\bar{\lambda}_{2}^{k} A_{2}^{-1} \operatorname{div}\left(\Delta j_{n 1 T}\right)+\frac{1}{\bar{\lambda}_{2}^{k}} .
$$


Setting

$$
\begin{aligned}
& b_{1,2}=1+\bar{\lambda}_{2}^{k} A_{2 T}^{-1} c_{2, \phi_{n}}^{\prime}, \\
& b_{2,2}=\bar{\lambda}_{2}^{k} A_{2 T}^{-1}, \\
& b_{3,2}=\frac{1}{\bar{\lambda}_{2}^{k}} .
\end{aligned}
$$

We deduce

$$
\Delta \phi_{n 1 T}=\frac{b_{2,1}}{b_{1,2}} \operatorname{div}\left(\Delta j_{n 1 T}\right)+\frac{b_{3,2}}{b_{1,2}} .
$$

The index $\mathrm{T}$ will be omitted. Now if we inject the above equation in (87), we have to solve only a system with one unknown $\Delta j_{n 1}$, which is:

$$
\begin{aligned}
& \sum_{T}\left\{\int_{T} \alpha_{2}\left(x, \phi^{k}, \phi_{n}^{k}\right) \Delta j_{n 1} v_{2} d x+\right. \\
& \left.\int_{T}\left[\frac{-N_{\phi_{n}}^{\prime}\left(x, \phi^{k}, \phi_{n}^{k}\right)}{q \mu_{n}(x) N\left(x, \phi^{k}, \phi_{n}^{k}\right)^{2}} j_{n}^{k} v_{2}+\operatorname{div}\left(v_{2}\right)\right] \frac{b_{2,2}}{b_{1,2}} \operatorname{div}\left(\Delta j_{n 1}\right) d x\right\}= \\
& \sum_{T}\left\{\int_{T}\left[\frac{N_{\phi_{n}}^{\prime}\left(x, \phi^{k}, \phi_{n}^{k}\right)}{q \mu_{n}(x) N\left(x, \phi^{k}, \phi_{n}^{k}\right)^{2}} j_{n}^{k} \cdot v_{2}+\operatorname{div}\left(v_{2}\right)\right] \frac{b_{3,2}}{b_{1,2}} d x .\right.
\end{aligned}
$$

Formally, the above system can be written as follows

$$
\bar{K}_{2} \Delta j_{n 1}=G_{2} .
$$

The operator $\mathrm{M}$ associated with the matrix $\bar{K}_{2}$ is of the form $\alpha I+\beta d i v+\gamma J$. the matrix $\bar{K}_{2}$ is then neither symmetric nor positive definite. But, thanks to a block Gaussian elimination it is only of order $N N \times N N$. The same Gaussian elimination can be used for the other equations and obtain relations similar to (90), i.e.

$$
\begin{aligned}
& \bar{K}_{1} \Delta D_{1}=G_{1} . \\
& \bar{K}_{3} \Delta j_{p 1}=G_{3} .
\end{aligned}
$$


Remark 4.2 The velocities $\left(\dot{\phi}_{n}^{k}, \mathrm{j}_{n}^{k}\right)$ can be updated within the Newton-Raphson's corrector loop. This case will be considered in the next variant of the above algorithm 2.

Remark 4.3 The nonlinear conjugate gradient methods can be used as correctors in stead of Newton-Raphson's type methods [52], [53], [54]. The combination of continuation and nonlinear conjugate gradient methods can be found in the paper of Glowinski and Keller (1985) [55], concerning the solution of certain nonlinear elliptic boundary values problems.

\subsection{Solution procedure for the nonlinear sub-systems}

In this section, we want to describe the procedure used to solve the nonlinear subsystems of the corrector step. At first, in order to minimize the distance from the prediction solution to the curve, we replace the constraints $\left(N_{3 i}\right)_{i=1,2,3}$ given above by the orthogonality constraints (see fig. $(11,12)$ ):

$$
N_{o i}\left(u_{i}, \lambda_{i}, s\right)=\omega_{i} \dot{u}_{i} \Delta u_{i}+\dot{\lambda}_{i} \Delta \lambda_{i}=0 .
$$

We can remark here that, if we use the latter constraint, the mathematical results given in theorem 3.2 remain valid.

Then, we apply the classical Newton-Raphson's method to solve the nonlinear subsystems (72),(73) and (74) with the orthogonality constraints (93). The initial guess is the prediction solution. Let $\mathrm{n}$ be the index of Newton-Raphson's iterations.

For all $n \geq 0$,

$$
\left(\phi^{k+1, n}, D^{k+1, n}, \lambda_{1}^{k+1, n}, \phi_{n}^{k+1, n}, j_{n}^{k+1, n}, \lambda_{2}^{k+1, n}, \phi_{p}^{k+1, n}, j_{p}^{k+1, n}, \lambda_{3}^{k+1, n}\right)
$$

given, we have to solve the following linear sub-systems 


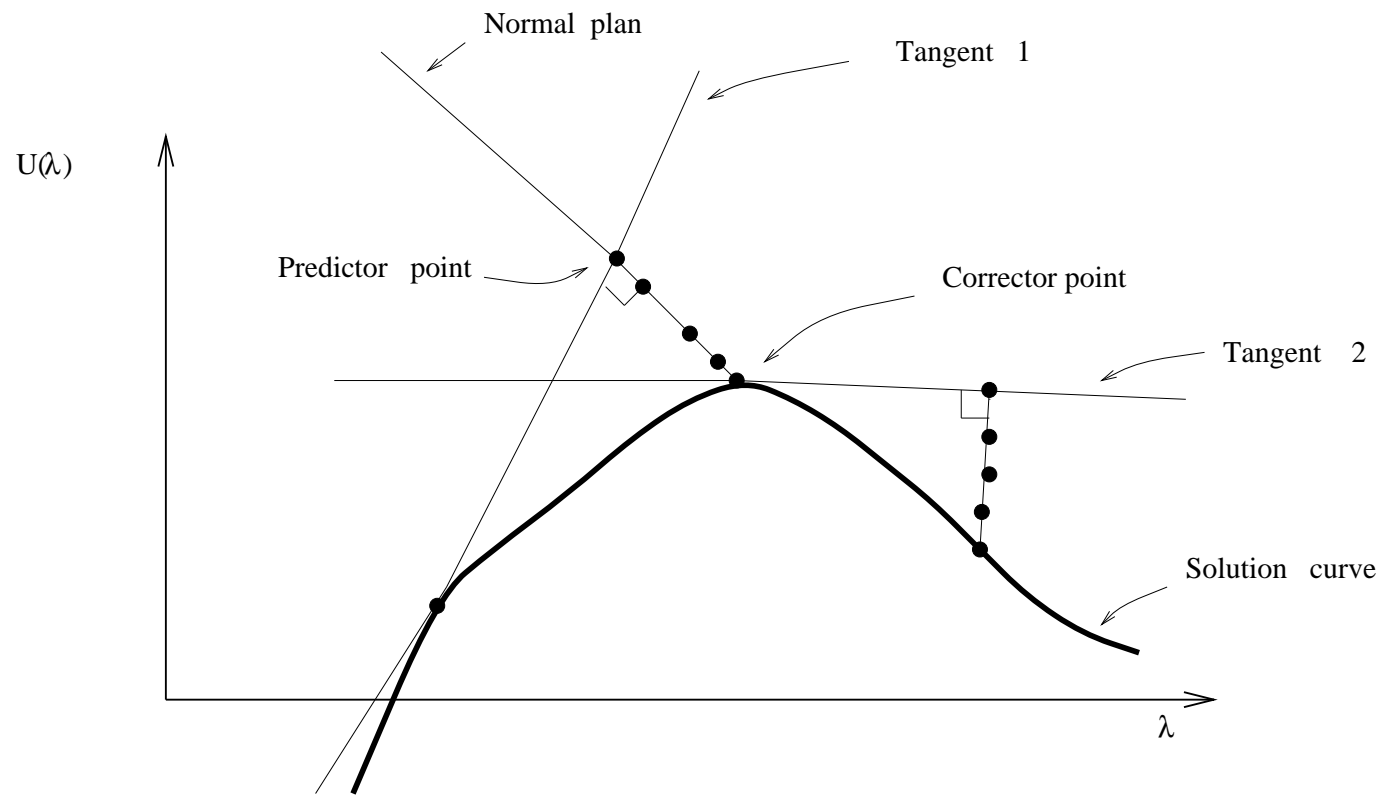

Figure 11: Algorithm 2: Arclength continuation type algorithm with orthogonality constraint. The suited path is orthogonal to the velocity vector. 


$$
\left\{\begin{array}{l}
\frac{\partial H_{11}\left(\phi^{k+1, n}, D^{k+1, n}, \lambda_{1}^{k+1, n}, \phi_{n}^{k}, \phi_{p}^{k}\right)}{\partial \phi} \Delta \phi+\frac{\partial H_{11}\left(\phi^{k+1, n}, D^{k+1, n}, \lambda_{1}^{k+1, n}, \phi_{n}^{k}, \phi_{p}^{k}\right)}{\partial D} \Delta D+ \\
\frac{\partial H_{11}\left(\phi^{k+1, n}, D^{k+1, n}, \lambda_{1}^{k+1, n}, \phi_{n}^{k}, \phi_{p}^{k}\right)}{\partial \lambda_{1}} \Delta \lambda_{1}=-H_{11}\left(\phi^{k+1, n}, D^{k+1, n}, \lambda_{1}^{k+1, n}, \phi_{n}^{k}, \phi_{p}^{k}\right), \\
\frac{\partial H_{12}\left(\phi^{k+1, n}, D^{k+1, n}, \lambda_{1}^{k+1, n}, \phi_{n}^{k}, \phi_{p}^{k}\right)}{\partial \phi} \Delta \phi+\frac{\partial H_{12}\left(\phi^{k+1, n}, D^{k+1, n}, \lambda_{1}^{k+1, n}, \phi_{n}^{k}, \phi_{p}^{k}\right)}{\partial D} \Delta D+ \\
\frac{\partial H_{12}\left(\phi^{k+1, n}, D^{k+1, n}, \lambda_{1}^{k+1, n}, \phi_{n}^{k}, \phi_{p}^{k}\right)}{\partial \lambda_{1}} \Delta \lambda_{1}=-H_{12}\left(\phi^{k+1, n}, D^{k+1, n}, \lambda_{1}^{k+1, n}, \phi_{n}^{k}, \phi_{p}^{k}\right), \\
\omega_{l_{1}} \dot{\phi}^{k} \Delta \phi+\omega_{p_{d}} \dot{D}^{k} \Delta D+\dot{\lambda}_{1}^{k} \Delta \lambda_{1}=0,
\end{array}\right.
$$

$$
\left\{\begin{array}{l}
\frac{\partial H_{21}\left(\phi_{n}^{k+1}, j_{n}^{k+1, n}, \lambda_{2}^{k+1, n}, \phi^{k+1}, \phi_{p}^{k}\right)}{\partial \phi_{n}} \Delta \phi_{n}+\frac{\partial H_{21}\left(\phi_{n}^{k+1}, j_{n}^{k+1, n}, \lambda_{2}^{k+1, n}, \phi^{k+1}, \phi_{p}^{k}\right)}{\partial j_{n}} \Delta j_{n}+ \\
\frac{\partial H_{21}\left(\phi_{n}^{k+1}, j_{n}^{k+1, n}, \lambda_{2}^{k+1, n}, \phi^{k+1}, \phi_{p}^{k}\right)}{\partial \lambda_{2}} \Delta \lambda_{2}=-H_{21}\left(\phi_{n}^{k+1}, j_{n}^{k+1, n}, \lambda_{2}^{k+1, n}, \phi^{k+1}, \phi_{p}^{k}\right), \\
\frac{\partial H_{22}\left(\phi_{n}^{k+1}, j_{n}^{k+1, n}, \lambda_{2}^{k+1, n}, \phi^{k+1}, \phi_{p}^{k}\right)}{\partial \phi_{n}} \Delta \phi_{n}+\frac{\partial H_{22}\left(\phi_{n}^{k+1}, j_{n}^{k+1, n}, \lambda_{2}^{k+1, n}, \phi^{k+1}, \phi_{p}^{k}\right)}{\partial j_{n}} \Delta j_{n}+ \\
\frac{\partial H_{22}\left(\phi_{n}^{k+1}, j_{n}^{k+1, n}, \lambda_{2}^{k+1, n}, \phi^{k+1}, \phi_{p}^{k}\right)}{\partial \lambda_{2}} \Delta \lambda_{2}=-H_{22}\left(\phi_{n}^{k+1}, j_{n}^{k+1, n}, \lambda_{2}^{k+1, n}, \phi^{k+1}, \phi_{p}^{k}\right), \\
\omega_{l_{2}} \dot{\phi}_{n}^{k} \Delta \phi_{n}+\omega_{p_{n}} \dot{j}_{n}^{k} \Delta j_{n}+\dot{\lambda}_{2}^{k} \Delta \lambda_{2}=0,
\end{array}\right.
$$




$$
\left\{\begin{array}{l}
\frac{\partial H_{31}\left(\phi_{p}^{k+1}, j_{p}^{k+1, n}, \lambda_{3}^{k+1, n}, \phi^{k+1}, \phi_{n}^{k+1}\right)}{\partial \phi_{p}} \Delta \phi_{p}+\frac{\partial H_{31}\left(\phi_{p}^{k+1}, j_{p}^{k+1, n}, \lambda_{3}^{k+1, n}, \phi^{k+1}, \phi_{n}^{k+1}\right)}{\partial j_{p}} \Delta j_{p}+ \\
\frac{\partial H_{31}\left(\phi_{p}^{k+1}, j_{p}^{k+1, n}, \lambda_{3}^{k+1, n}, \phi^{k+1}, \phi_{n}^{k+1}\right)}{\partial \lambda_{3}} \Delta \lambda_{3}=-H_{31}\left(\phi_{p}^{k+1}, j_{p}^{k+1, n}, \lambda_{3}^{k+1, n}, \phi^{k+1}, \phi_{n}^{k+1}\right), \\
\frac{\partial H_{32}\left(\phi_{p}^{k+1}, j_{p}^{k+1, n}, \lambda_{3}^{k+1, n}, \phi^{k+1}, \phi_{n}^{k+1}\right)}{\partial \phi_{p}} \Delta \phi_{p}+\frac{\partial H_{32}\left(\phi_{p}^{k+1}, j_{p}^{k+1, n}, \lambda_{3}^{k+1, n}, \phi^{k+1}, \phi_{n}^{k+1}\right)}{\partial j_{p}} \Delta j_{p}+ \\
\frac{\partial H_{32}\left(\phi_{p}^{k+1}, j_{p}^{k+1, n}, \lambda_{3}^{k+1, n}, \phi^{k+1}, \phi_{n}^{k+1}\right)}{\partial \lambda_{3}} \Delta \lambda_{3}=-H_{32}\left(\phi_{p}^{k+1}, j_{p}^{k+1, n}, \lambda_{3}^{k+1, n}, \phi^{k+1}, \phi_{n}^{k+1}\right), \\
\omega_{l_{3}} \dot{\phi}_{p}^{k} \Delta \phi_{p}+\omega_{p_{p}} \dot{j}_{p}^{k} \Delta j_{p}+\dot{\lambda}_{3}^{k} \Delta \lambda_{3}=0,
\end{array}\right.
$$

where

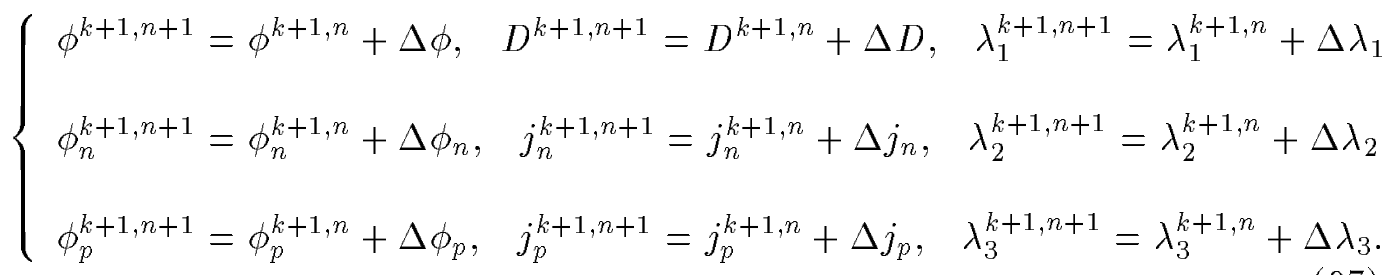

To solve these systems, we denote

$$
\left\{\begin{array}{c}
H_{1 i}=H_{1 i}\left(\phi^{k+1, n}, D^{k+1, n}, \lambda_{1}^{k+1, n}, \phi_{n}^{k}, \phi_{p}^{k}\right) \quad i=1,2, \\
H_{2 i}=H_{2 i}\left(\phi_{n}^{k+1}, j_{n}^{k_{1}, n}, \lambda_{2}^{k+1, n}, \phi^{k+1}, \phi_{p}^{k}\right) \quad i=1,2, \\
H_{3 i}=H_{3 i}\left(\phi_{p}^{k+1}, j_{p}^{k_{1}, n}, \lambda_{3}^{k+1, n}, \phi^{k+1}, \phi_{n}^{k+1}\right) \quad i=1,2, \\
K_{1}=\left(\begin{array}{cc}
\frac{\partial H_{11}}{\partial \phi} & \frac{\partial H_{11}}{\partial D} \\
\frac{\partial H_{12}}{\partial \phi} & \frac{\partial H_{12}}{\partial D}
\end{array}\right),
\end{array}\right.
$$




$$
\begin{gathered}
K_{2}=\left(\begin{array}{ll}
\frac{\partial H_{21}}{\partial \phi_{n}} & \frac{\partial H_{21}}{\partial j_{n}} \\
\frac{\partial H_{22}}{\partial \phi_{n}} & \frac{\partial H_{22}}{\partial j_{n}}
\end{array}\right), \\
K_{3}=\left(\begin{array}{ll}
\frac{\partial H_{31}}{\partial \phi_{p}} & \frac{\partial H_{31}}{\partial j_{p}} \\
\frac{\partial H_{32}}{\partial \phi_{p}} & \frac{\partial H_{32}}{\partial j_{p}}
\end{array}\right),
\end{gathered}
$$

As a formal procedure for solving the linear sub-systems (94), (95) and (96) we use the so-called BORDERING ALGORITHM, see [34], [56] :

\section{1) Poisson's equation}

$$
\text { Solve }\left\{\begin{array}{l}
a) \quad K_{1}\left(\Delta \phi_{0}, \Delta D_{0}\right)=-H_{1} \\
b) \quad K_{1}\left(\Delta \phi_{1}, \Delta D_{1}\right)=-\frac{\partial H_{1}}{\partial \lambda_{1}} .
\end{array}\right.
$$

Set :
a) $\Delta \lambda_{1}=\frac{-\left(\omega_{l_{1}} \dot{\phi} \cdot \Delta \phi_{0}+\omega_{p_{d}} \dot{D} \cdot \Delta D_{0}\right)}{\omega_{l_{1}} \dot{\phi} \cdot \Delta \phi_{1}+\omega_{p_{d}} \dot{D} \cdot \Delta D_{1}+\dot{\lambda}_{1}}$,
b) $\Delta \phi=\Delta \phi_{0}+\Delta \lambda_{1} \Delta \phi_{1}, \quad \Delta D=\Delta D_{0}+\Delta \lambda_{1} \Delta D_{1}$.

\section{2) Electron transport equations}

$$
\text { Solve }\left\{\begin{array}{l}
\text { a) } \quad K_{2}\left(\Delta \phi_{n 0}, \Delta j_{n 0}\right)=-H_{2} \\
b) \quad K_{2}\left(\Delta \phi_{n 1}, \Delta j_{n 1}\right)=-\frac{\partial H_{2}}{\partial \lambda_{2}} .
\end{array}\right.
$$

Set :
a) $\Delta \lambda_{2}=\frac{-\left(\omega_{l_{2}} \dot{\phi}_{n} \cdot \Delta \phi_{n 0}+\omega_{p_{n}} \dot{j}_{n} \cdot \Delta j_{n 0}\right)}{\omega_{l_{2}} \dot{\phi}_{n} \cdot \Delta \phi_{n 1}+\omega_{p_{n}} \dot{j}_{n} \cdot \Delta j_{n 1}+\dot{\lambda}_{2}}$
b) $\Delta \phi_{n}=\Delta \phi_{n 0}+\Delta \lambda_{2} \Delta \phi_{n 1}, \quad \Delta j_{n}=\Delta j_{n 0}+\Delta \lambda_{2} \Delta j_{n 1}$. 


\section{3) Hole transport equations}

$$
\text { Solve }\left\{\begin{array}{l}
\text { a) } \quad K_{3}\left(\Delta \phi_{p 0}, \Delta j_{p 0}\right)=-H_{3} \\
b) \quad K_{3}\left(\Delta \phi_{p 1}, \Delta j_{p 1}\right)=-\frac{\partial H_{3}}{\partial \lambda_{3}} .
\end{array}\right.
$$

Set :

$$
\begin{aligned}
& \text { a) } \Delta \lambda_{3}=\frac{-\left(\omega_{l} \dot{\phi}_{p} \cdot \Delta \phi_{p 0}+\omega_{p_{p}} \dot{j}_{p} \cdot \Delta j_{p 0}\right)}{\omega_{l_{3}} \dot{\phi}_{p} \cdot \Delta \phi_{p 1}+\omega_{p_{p}} \dot{j}_{p} \cdot \Delta j_{p 1}+\dot{\lambda}_{3}} \\
& \text { b) } \Delta \phi_{p}=\Delta \phi_{p 0}+\Delta \lambda_{3} \Delta \phi_{p 1}, \quad \Delta j_{p}=\Delta j_{p 0}+\Delta \lambda_{3} \Delta j_{p 1} .
\end{aligned}
$$

Let $\mathcal{A}_{1}$ (resp. $\mathcal{A}_{2,3}$ ) denotes the matrix associated with the sub-system (94) (resp. $(95),(96))$.

Since the matrix $K_{1}\left(\operatorname{resp} . K_{2,3}\right)$ is nonsingular then $\left(\Delta \phi_{0,1}, \Delta D_{0,1}\right)\left(\operatorname{resp} .\left(\Delta \phi_{n, p, 0,1}\right.\right.$, $\left.\left.\Delta j_{n, p, 0,1}\right)\right)$ is well defined and the denominator

$$
\omega_{l_{1}} \dot{\phi} \cdot \Delta \phi_{1}+\omega_{p_{d}} \dot{D} \cdot \phi_{1}+\dot{\lambda}_{1}
$$

(resp. $\omega_{l_{2,3}} \dot{\phi}_{n, p} \cdot \Delta \phi_{n, p, 1}+\omega_{p_{n, p}} \dot{j}_{n, p} . \phi_{n, p, 1}+\dot{\lambda}_{2,3}$ ) is just the Schur complement of $K_{1}$ (resp. $K_{2,3}$ ) in $\mathcal{A}_{1}\left(\operatorname{resp} . \mathcal{A}_{2,3}\right)$ [33].

The quantities $\Delta \lambda_{1,2,3}$ are well defined since $\mathcal{A}_{1,2,3}$ are nonsingular by the results given above.

The bordering algorithm is stopped for each equation 1), 2) and 3) when

$$
\begin{aligned}
& \max \left(\frac{\|\Delta \phi\|_{l^{1}}}{\left\|\phi^{k+1, n+1}\right\|_{l^{1}}}, \frac{\|\Delta D\|_{l^{1}}}{\left\|D^{k+1, n+1}\right\|_{l^{1}}}, \frac{\left\|\Delta \lambda_{1}\right\|_{l^{1}}}{\left\|\lambda_{1}^{k+1, n+1}\right\|_{l^{1}}}\right) \leq \varepsilon_{1}, \\
& \max \left(\frac{\left\|\Delta \phi_{n}\right\|_{l^{1}}}{\left\|\phi_{n}^{k+1, n+1}\right\|_{l^{1}}}, \frac{\left\|\Delta j_{n}\right\|_{l^{1}}}{\left\|j_{n}^{k+1, n+1}\right\|_{l^{1}}}, \frac{\left\|\Delta \lambda_{2}\right\|_{l^{1}}}{\left\|\lambda_{2}^{k+1, n+1}\right\|_{l^{1}}}\right) \leq \varepsilon_{1}, \\
& \max \left(\frac{\left\|\Delta \phi_{p}\right\|_{l^{1}}}{\left\|\phi_{p}^{k+1, n+1}\right\|_{l^{1}}}, \frac{\left\|\Delta j_{p}\right\|_{l^{1}}}{\left\|j_{p}^{k+1, n+1}\right\|_{l^{1}}}, \frac{\left\|\Delta \lambda_{3}\right\|_{l^{1}}}{\left\|\lambda_{3}^{k+1, n+1}\right\|_{l^{1}}}\right) \leq \varepsilon_{1},
\end{aligned}
$$

where $\varepsilon_{1}$ is sufficiently small.

The choice of $\varepsilon_{1}$ is machine precision dependent. In double precision, we have taken $\varepsilon_{1} \sim 10^{-8}$. In quadruple precision, we have taken $\varepsilon_{1} \sim 10^{-12}$. Some technical problems remain in double precision for the appreciation of the convergence in NewtonRaphson corrector steps; residual values (specially for one of the two dual variables $j_{n}$ or $j_{p}$ ) may remain beyond $\sim 10^{-6}$ in some applications. These technical problems may be related with our choice of convergence test. 


\subsection{On the solution of the linear systems}

Using the same procedure (local Gaussian elimination) as in (86)- (89) we can reduce the matrices $K_{1}, K_{2}$ and $K_{3}$ which are $(N N+N E) \times(N N+N E)$ to matrices $\bar{K}_{1}, \bar{K}_{2}$ and $\bar{K}_{3}$ whose order is only $N N \times N N$. Hence, the solution procedure is less expensive. The matrices $\bar{K}_{1}, \bar{K}_{2}$ and $\bar{K}_{3}$, are sparse. Since our (MFE) is of lowest order, only five elements in each line of these matrices are not zero. The matrix $\bar{K}_{1}$ is symmetric and positive definite and a good choice is to factor $\bar{K}_{1}$ and solve the associated system by Cholesky method.

The matrices $\bar{K}_{2}$ and $\bar{K}_{3}$ are neither symmetric nor positive definite, then a Gauss elimination technique (without pivoting) is chosen. The ordering technique of the unknowns is used in order to reduce the skyline profile of matrices.

Remark 4.4 If $\bar{K}_{1}, \bar{K}_{2}$ or $\bar{K}_{3}$ happen to be singular (time steps are too large or some limit or bifurcation points are touched) then results of some accuracy will be obtained only if a reasonable pivoting strategy is used.

Remark 4.5 When the systems are too large (in higher dimension or more gridpoints) a conjugate gradient type solver must be combined with the algorithm.

\subsection{Operational count for the algorithm 2}

To show that our arclength continuation algorithm is not more expensive neither in time nor in space (at less) than our nonlinear implicit scheme, without continuation procedures as presented in [29], we give the operational count for the full system.

In algorithm 2, there are two essential points : calculations of the velocities at each continuation step and the bordering algorithm for all corrector steps.

\section{1) On the computation of the velocities at each continuation step :}

- One $L L^{t}$ - factorization of $N N \times N N$ matrix $\bar{K}_{1}$ to compute the intermediate dual variable.

- Two $L U$-factorizations of $N N \times N N$ matrices $\bar{K}_{2,3}$ to compute the intermediate dual variables.

- Three inner products to form the intermediate primal variables.

- Six inner products to form the velocity vectors. 


\section{2) On the bordering algorithm for each continuation step :}

- One $L L^{t}$ - factorization of $N N \times N N$ matrix $\left(\bar{K}_{1}\right)$, two backsolves with the same factored matrix to compute the intermediate dual variables.

- Two $L U$-factorizations of $N N \times N N$ matrices $\left(\bar{K}_{2,3}\right)$, four backsolves with the factored matrices (for each transport equations, the two backsolves are with the same factored matrix) to compute the intermediate dual variables.

- Three inner products to form the intermediate primal variables.

\subsection{Algorithm 3 :}

Another algorithm can be derived as a variant of the algorithm 2, in which the velocity update is done only within the Newton-Raphson's corrector steps (except at first continuation iteration), see figure 12. The detailed algorithm is as follows :

$0]$ Initialization :

1] Continuation loop :

For only the first iteration,

- compute and factor the tangent operators :

$$
\bar{K}_{1}, \bar{K}_{2}, \bar{K}_{3}
$$

- compute $G_{1}, G_{2}$ and $G_{3}$, which depend of the line derivative $\left(\frac{\partial H_{i j}}{\partial \lambda_{i}}\right)_{i=1,2,3}^{j=1,2}$. The operators $\bar{K}_{1,2,3} \quad G_{1,2,3}$ and the coefficients $b_{i, j} \quad i, j=1,2,3$ are given by a procedure similar to (84)-(89),

- solve the linear systems :

$$
\bar{K}_{1} \Delta D_{1}=G_{1}, \quad \bar{K}_{2} \Delta j_{n 1}=G_{2}, \quad \bar{K}_{3} \Delta j_{p 1}=G_{3},
$$

- set

$$
\begin{aligned}
\Delta \phi_{1} & =\frac{b_{2,1}}{b_{1,1}} \operatorname{div}\left(\Delta D_{1}\right)+\frac{b_{3,1}}{b_{1,1}} \\
\Delta \phi_{n 1} & =\frac{b_{2,2}}{b_{1,2}} \operatorname{div}\left(\Delta j_{n 1}\right)+\frac{b_{3,2}}{b_{1,2}}
\end{aligned}
$$




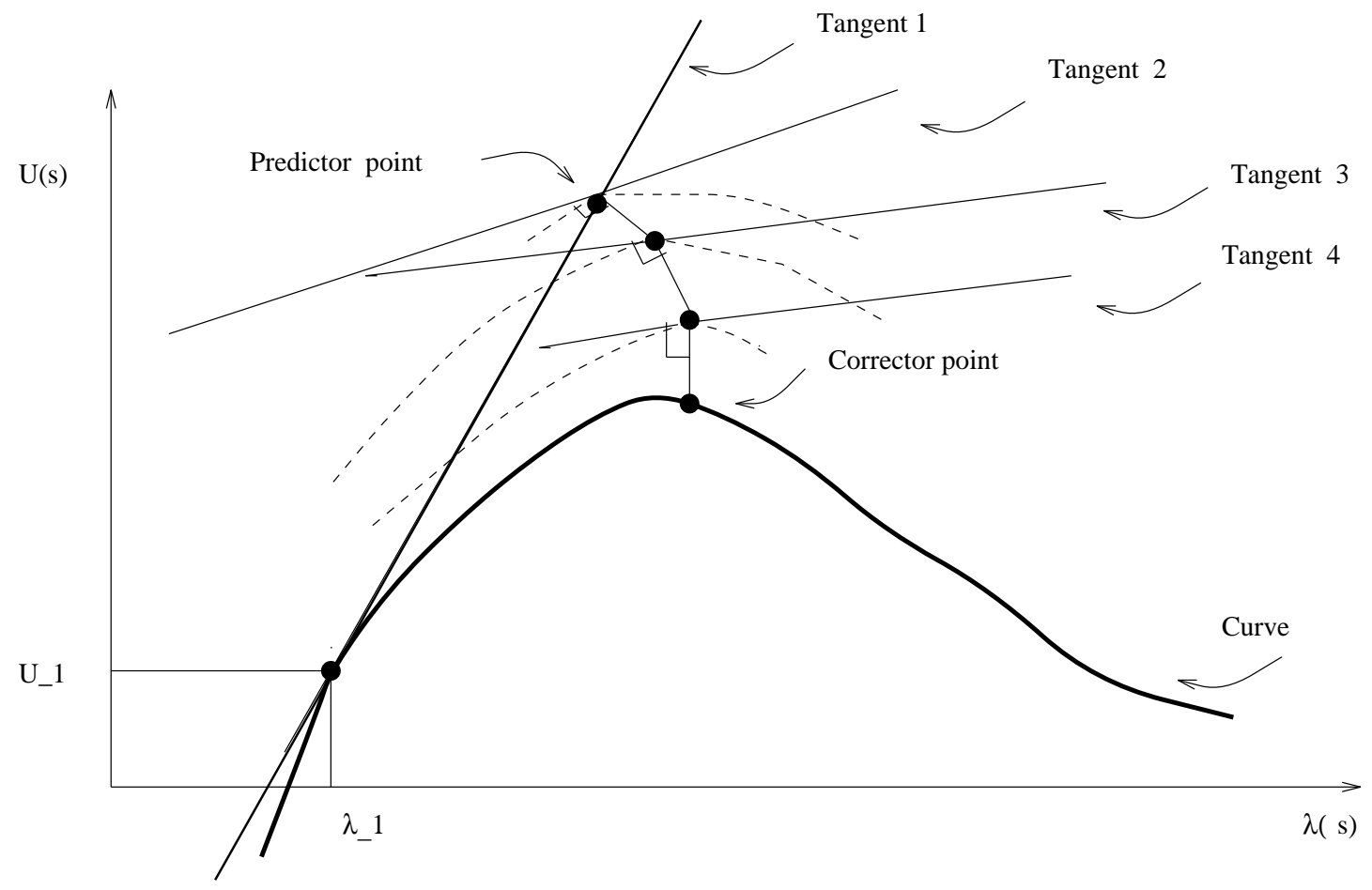

Figure 12: Algorithm 3: Arclength continuation type algorithm. In this case, the velocity update is done within the Newton-Raphson's corrector steps. Then, the distance to the solution curve is minimized for each corrector steps. The number of corrector steps will be then less than the number in algorithm 2. 


$$
\Delta \phi_{p 1}=\frac{b_{2,3}}{b_{1,3}} \operatorname{div}\left(\Delta j_{p 1}\right)+\frac{b_{3,3}}{b_{1,3}}
$$

- set

$$
\begin{gathered}
\dot{\lambda}_{1}=\frac{1}{\sqrt{\omega_{l_{1}}\left\|\Delta \phi_{1}\right\|_{l^{2}}^{2}+\omega_{p_{d}}\left\|\Delta D_{1}\right\|_{l^{2}}^{2}+1}}, \\
\dot{\lambda}_{2}=\frac{1}{\sqrt{\omega_{l_{2}}\left\|\Delta \phi_{n 1}\right\|_{l^{2}}^{2}+\omega_{p_{n}}\left\|\Delta j_{n 1}\right\|_{l^{2}}^{2}+1}}, \\
\quad \frac{1}{\sqrt{\omega_{l_{3}}\left\|\Delta \phi_{p 1}\right\|_{l^{2}}^{2}+\omega_{p_{p}}\left\|\Delta j_{p 1}\right\|_{l^{2}}^{2}+1}}, \\
(\dot{\phi}, \dot{D})=\left(\Delta \phi_{1}, \Delta D_{1}\right) \dot{\lambda}_{1}, \\
\left(\dot{\phi}_{n}, \dot{j}_{n}\right)=\left(\Delta \phi_{n 1}, \Delta j_{n 1}\right) \dot{\lambda}_{2}, \\
\left(\dot{\phi}_{p}, \dot{j}_{p}\right)=\left(\Delta \phi_{p 1}, \Delta j_{p 1}\right) \dot{\lambda}_{3} .
\end{gathered}
$$

1.2] Euler's predictor step :

- set

$$
\left(\phi, D, \lambda_{1}\right)=\left(\phi, D, \lambda_{1}\right)+\Delta s_{1}\left(\dot{\phi}, \dot{D}, \dot{\lambda}_{1}\right)
$$

- set

$$
\begin{gathered}
\left(\phi_{n}, j_{n}, \lambda_{2}\right)=\left(\phi_{n}, j_{n}, \lambda_{2}\right)+\Delta s_{2}\left(\dot{\phi}_{n}, \dot{j}_{n}, \dot{\lambda}_{2}\right), \\
\left(\phi_{p}, j_{p}, \lambda_{3}\right)=\left(\phi_{p}, j_{p}, \lambda_{3}\right)+\Delta s_{3}\left(\dot{\phi}_{p}, \dot{j}_{p}, \dot{\lambda}_{3}\right) .
\end{gathered}
$$

1.3] Newton-Raphson's corrector loop :

\subsection{1) Poisson's equation :}

1.3.1.1) Stiffness and velocity updates : For each iteration

- compute and factor the tangent operator : $\bar{K}_{1}$,

- compute $G_{11}, G_{12}$ which are obtained by Gaussian elimination applied to subsystem (94). $G_{11}$ depends of the line derivative $\frac{\partial H_{11}}{\partial \lambda_{1}}$. 
- solve the linear system :

$$
\bar{K}_{1} \Delta D_{1}=G_{11}
$$

- set

$$
\Delta \phi_{1}=\frac{b_{2,1}}{b_{1,1}} \operatorname{div}\left(\Delta D_{1}\right)+\frac{b_{3,1}}{b_{1,1}}
$$

- set

$$
\begin{gathered}
\dot{\lambda}_{1}=\frac{1}{\sqrt{\omega_{l_{1}}\left\|\Delta \phi_{1}\right\|_{l^{2}}^{2}+\omega_{p_{d}}\left\|\Delta D_{1}\right\|_{l^{2}}^{2}+1}}, \\
(\dot{\phi}, \dot{D})=\left(\Delta \phi_{1}, \Delta D_{1}\right) \dot{\lambda}_{1} .
\end{gathered}
$$

1.3.1.2) solution increment update :

- solve the system :

$$
\bar{K}_{1} \Delta D_{0}=G_{12}
$$

- set

$$
\Delta \phi_{0}=\frac{b_{2,1}}{b_{1,1}} \operatorname{div}\left(\Delta D_{0}\right)+\frac{b_{3,1}}{b_{1,1}}
$$

- set

$$
\Delta \lambda_{1}=\frac{-\left(\omega_{l_{1}} \dot{\phi} \cdot \Delta \phi_{0}+\omega_{p_{d}} \dot{D} \cdot \Delta D_{0}\right)}{\omega_{l_{1}} \dot{\phi} \cdot \Delta \phi_{1}+\omega_{p_{d}} \dot{D} \cdot \Delta D_{1}+\dot{\lambda}_{1}},
$$

- set

$$
\begin{gathered}
\Delta \phi=\Delta \phi_{0}+\Delta \lambda_{1} \Delta \phi_{1}, \\
\Delta D=\Delta D_{0}+\Delta \lambda_{1} \Delta D_{1}, \\
\phi=\phi+\Delta \phi, \\
D=D+\Delta D, \\
\lambda_{1}=\lambda_{1}+\Delta \lambda_{1} .
\end{gathered}
$$

\subsubsection{3) convergence test :}

if

$$
\max \left(\frac{\|\Delta \phi\|_{l^{1}}}{\left\|\phi^{k+1, n+1}\right\|_{l^{1}}}, \frac{\|\Delta D\|_{l^{1}}}{\left\|D^{k+1, n+1}\right\|_{l^{1}}}, \frac{\left\|\Delta \lambda_{1}\right\|_{l^{1}}}{\left\|\lambda_{1}^{k+1, n+1}\right\|_{l^{1}}}\right) \leq \varepsilon_{2},
$$


stop the Newton-Raphson's loop and go to the next equation.

\subsection{2) electron transport equations :}

1.3.2.1) Stiffness and velocity updates :

For each iteration, in the same way as for the Poisson's equation,

- compute and factor the tangent operator : $\bar{K}_{2}$,

- compute $G_{21}, G_{22}$ which are obtained by Gaussian elimination applied to subststem (95). $G_{21}$ depends of the line derivative $\left(\frac{\partial H_{21}}{\partial \lambda_{2}}\right)$,

- solve the linear system :

$$
\bar{K}_{2} \Delta j_{n 1}=G_{21}
$$

- set

$$
\Delta \phi_{n 1}=\frac{b_{2,2}}{b_{1,2}} \operatorname{div}\left(\Delta j_{n 1}\right)+\frac{b_{3,2}}{b_{1,2}}
$$

- set

$$
\begin{gathered}
\dot{\lambda}_{2}=\frac{1}{\sqrt{\omega_{l_{2}}\left\|\Delta \phi_{n 1}\right\|_{l^{2}}^{2}+\omega_{p_{n}}\left\|\Delta j_{n 1}\right\|_{l^{2}}^{2}+1}}, \\
\left(\dot{\phi}_{n}, \dot{j}_{n}\right)=\left(\Delta \phi_{n 1}, \Delta j_{n 1}\right) \dot{\lambda}_{2} .
\end{gathered}
$$

1.3.2.2) solution increment update :

- solve the system :

$$
\bar{K}_{2} \Delta j_{n 0}=G_{22} .
$$

- set

$$
\Delta \phi_{n 0}=\frac{b_{2,2}}{b_{1,2}} \operatorname{div}\left(\Delta j_{n 0}\right)+\frac{b_{3,2}}{b_{1,2}}
$$

- set

$$
\Delta \lambda_{2}=\frac{-\omega_{l_{2}} \dot{\phi}_{n} \cdot \Delta \phi_{n 0}+\omega_{p_{n}} \dot{j}_{n} \cdot \Delta j_{n 0}}{\omega_{l_{2}} \dot{\phi}_{n} \cdot \Delta \phi_{n 1}+\omega_{p_{n}} \dot{j}_{n} \cdot \Delta j_{n 1}+\dot{\lambda}_{n 1}}
$$


- set

$$
\begin{gathered}
\Delta \phi_{n}=\Delta \phi_{n 0}+\Delta \lambda_{2} \Delta \phi_{n 1}, \\
\Delta j_{n}=\Delta j_{n 0}+\Delta \lambda_{2} \Delta j_{n 1}, \\
\phi_{n}=\phi_{n}+\Delta \phi_{n}, \\
j_{n}=j_{n}+\Delta j_{n}, \\
\lambda_{2}=\lambda_{2}+\Delta \lambda_{2} .
\end{gathered}
$$

\subsubsection{3) convergence test :}

if $\max \left(\frac{\left\|\Delta \phi_{n}\right\|_{l^{1}}}{\left\|\phi^{k+1, n+1}\right\|_{l^{1}}}, \frac{\left\|\Delta j_{n}\right\|_{l^{1}}}{\left\|j_{n}^{k+1, n+1}\right\|_{l^{1}}}, \frac{\left\|\Delta \lambda_{2}\right\|_{l^{1}}}{\left\|\lambda_{2}^{k+1, n+1}\right\|_{l^{1}}}\right) \leq \varepsilon_{1}$, stop the Newton-Raphson's loop and go to the final equation.

\subsection{3) hole transport equations :}

Stiffness and velocities updates, solution increment update and convergence test are similar to those in electron transport equation.

1.4] steplength adaptation and convergence test : Setting

$$
\begin{gathered}
e_{1}=\max \left(\frac{\left\|\phi^{k+1}-\phi^{k}\right\|_{l^{1}}}{\left\|\phi^{k+1}\right\|_{l^{1}}}, \frac{\left\|D^{k+1}-D^{k}\right\|_{l^{1}}}{\left\|D^{k+1}\right\|_{l^{1}}}\right), \\
e_{2}=\max \left(\frac{\left\|\phi_{n}^{k+1}-\phi_{n}^{k}\right\|_{l^{1}}}{\left\|\phi_{n}^{k+1}\right\|_{l^{1}}}, \frac{\left\|j_{n}^{k+1}-\mathrm{J}_{n}^{k}\right\|_{l^{1}}}{\left\|j_{n}^{k+1}\right\|_{l^{1}}}\right), \\
e_{3}=\max \left(\frac{\left\|\phi_{p}^{k+1}-\phi_{p}^{k}\right\|_{l^{1}}}{\left\|\phi_{p}^{k+1}\right\|_{l^{1}}}, \frac{\left\|j_{p}^{k+1}-j_{p}^{k}\right\|_{l^{1}}}{\left\|j_{p}^{k+1}\right\|_{l^{1}}}\right),
\end{gathered}
$$

if $\max \left(e_{1}, e_{2}, e_{3}\right)<\varepsilon_{2}$ then stop the arclength continuation loop, if not then choose new steplengths $\Delta s_{1}, \Delta s_{2}$ and $\Delta s_{3}$ and repeat the process.

\section{END ALGORITHM 3.}


Remark 4.6 In this algorithm, the factorization of the matrices is done only in the Newton-Raphson's corrector loop. Since the bulk of the computations occur in determining the factorization it follows that the algorithm 3 is less expensive than the algorithm 2. On the other hand, since the velocity update is done in NewtonRaphson's iterations, we minimize for such iterations the distance to solution curve, see figure 12. Hence, the algorithm 3 is theoretically faster and more robust than the algorithm 2. Numerical results will confirm this remark.

Remark 4.7 When dealing with limit or bifurcation points, the sign of the velocities $\dot{\lambda}_{1,2,3}$ can't be constant for all iterations, see figure 13 . In this cases, it may be determined as follows. For electron transport equations, we have

$$
\operatorname{sign}\left(\dot{\lambda}_{2}\right)=\operatorname{sign}\left(\omega_{l 2} \dot{\phi}_{n} \cdot \Delta \phi_{n 1}+\omega_{p d} \dot{j}_{n} \cdot \Delta j_{n 1}+\dot{\lambda}_{2}\right) \text {. }
$$

The sign of $\dot{\lambda}_{1,3}$, associated with the other equations, can be determined in the same way.

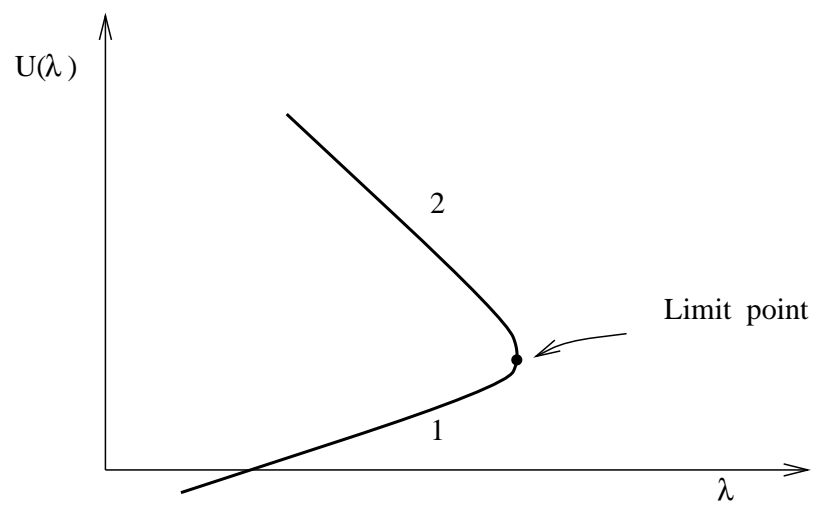

Figure 13: Velocity sign. In part $1, \dot{\lambda}_{1,2,3}$ are positive, but in part $2, \dot{\lambda}_{1,2,3}$ are negative. In limit point, $\dot{\lambda}_{1,2,3}=0$.

\section{Steplength adaptation for the predictor step}

One key point of the previous continuation type algorithms is the adaptation of the steplengths $\Delta s_{1,2,3}$ to the nonlinearity of problem. The convergence of the al- 
gorithms 2, 3 were carried out under the assumption that the steplengths $\Delta s_{1,2,3}$ were uniformally constants throughout the process. This is of course not efficient for any practical implementation. In our case, for example, since we want to obtain the stationary solution, it is desirable that the steplength become as large as possible. In the previous presentations of our algorithms 2,3 , we did not indicate any means of choosing the steplength. In fact, from our numerical experiments, the choice of these parameters may be critical for the speed of convergence of Newton-Raphson's algorithm. The choice of adaptive strategy is, of course, more problem dependent. It also depends upon the accuracy with which it is desired to numerically trace a solution curves, and the desired safety with which we want to follow $H_{1,2,3}^{-1}(0)$. In any case, an efficient algorithm for this task needs to incorporate an automatic strategy for controlling the steplength. In this way, the general opinion is that it is preferable to exploit the contractive properties of the zero set $H_{1,2,3}^{-1}(0)$ relative to such iterative methods as those of Newton-Raphson's type. In this paper, we shall outline some possible automatic steplength strategies, based upon a posteriori estimates for the performance of the corrector process, [37], [57], [58]. Given the number $n$ of the Newton-Raphson's iterations for the corrector step, and given an estimate $a$, which appears "ideal" to obtain the solution, the steplengths $\Delta s_{1,2,3}$, for the next predictor step, can be estimated by the following automatic strategies :

\section{i) Strategy 1}

$$
\Delta s_{1,2,3}=(a / n)^{\alpha} \Delta s_{1,2,3}
$$

\section{ii) Strategy 2}

$$
\Delta s_{1,2,3}=2^{(a-n) / b} \Delta s_{1,2,3} .
$$

The choice of $a, b$, and $\alpha$ depends upon the accuracy and the manner with which it is desired to trace numerically the solution curve.

For our numerical experiences, we have taken

$$
a \in[8,12], \quad b<a,(b \sim a / 2), \quad \alpha=1 / 2 .
$$

For example, with $a=12$ and $b=6$ in (113), the maximum value for the multiplicative factor applied to $\Delta s_{1,2,3}$ is 4 . If the number of Newton steps is equal to the estimated value $a$, then the same $\Delta s_{1,2,3}$ will be taken for the next continuation 
step. If the number $n$ is bigger than $a$ then $\Delta s_{1,2,3}$ will decrease for the next step. The steplengths $\Delta s_{1,2,3}$ given by the strategy 2 are larger than those given by strategy 1 . Then, when the nonlinearity is strong (high applied potential, $g_{2}=2$ volts, for an heterojunction diode for example) it may be preferable to use the first strategy.

For a more rigorous control of steplengths, there are several factors which ought to be considered in governing the steplength :

\section{1) The contraction rate :}

$$
k\left(u_{i}, \Delta s_{i}\right)=\frac{\|\left[H_{i}^{\prime}\left(w_{i}\left(s_{i}+\Delta s_{i}\right)\right)\right]^{-1} H_{i}\left(\left(w_{i}\left(s_{i}+\Delta s_{i}\right)\right) \|_{0}\right.}{\|\left[H_{i}^{\prime}\left(v_{i}\left(s_{i}+\Delta s_{i}\right)\right)\right]^{-1} H_{i}\left(\left(v_{i}\left(s_{i}+\Delta s_{i}\right)\right) \|_{0}\right.}, \quad i=1,2,3,
$$

where $v_{i}\left(s_{i}+\Delta s_{i}\right)$ is the predicted point, and $w_{i}\left(s_{i}+\Delta s_{i}\right)$ is the first corrected point. Then the contraction rate, $k\left(u_{i}, \Delta s_{i}\right)$ is the quotient of the two successive Newton-Raphson's steps.

Since Newton-Raphson's method is locally quadratically convergent, it is plain that $k\left(u_{i}, \Delta s_{i}\right)$ will decrease (and hence the Newton-Raphson's method will become faster) if $\Delta s_{i}$ decrease and hence $v_{i}\left(s_{i}+\Delta s_{i}\right)$ approaches $H_{i}^{-1}(0) ; i=1,2,3$.

\section{2) The first corrector steplength}

$$
\delta\left(u_{i}, \Delta s_{i}\right)=\|\left[H_{i}^{\prime}\left(v_{i}\left(s_{i}+\Delta s_{i}\right)\right)\right]^{-1} H_{i}\left(\left(v_{i}\left(s_{i}+\Delta s_{i}\right)\right) \|_{0}\right.
$$

which approximates the distance to the curve.

\section{3) The angle between two consecutive steps :}

This is a measure of the curvature, see figure 14 .

The following three estimates are based upon asymptotic estimates in the mentality of initial value solvers. In a next paper [59], we will study in detail, from mathematical and numerical viewpoint, these factors and we will introduce other strategies (based upon an error model for the corrector iteration, and interval arithmetic thechniques).

For the numerical results presented here, we limite ourselves to the automatic strategies given by (112), or (113). 


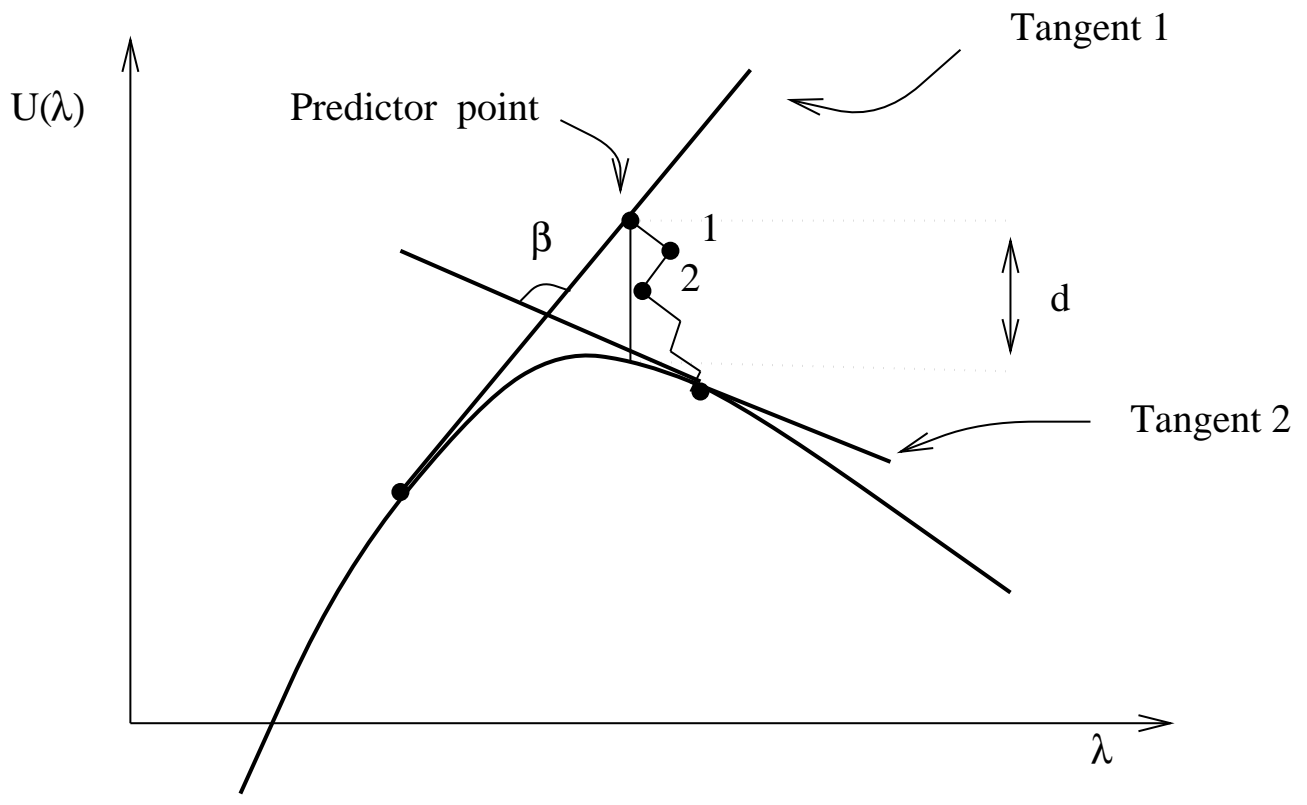

Figure 14: Automatic steplength adatptation. The angle $\beta$ is a measure of the curvature. We have $\beta \in[0, \pi]$. When the nonlinearity is strong, the angle $\beta$ tends to zero, and when the nonlinearity is weak $\beta$ tends to $\pi$. The quantity $d$ is an approximation of the distance to the curve. The points 1, and 2 are the two first points in the corrector process. 


\section{Numerical results}

\subsection{Heterojunction diode}

\subsubsection{Description of the structure}

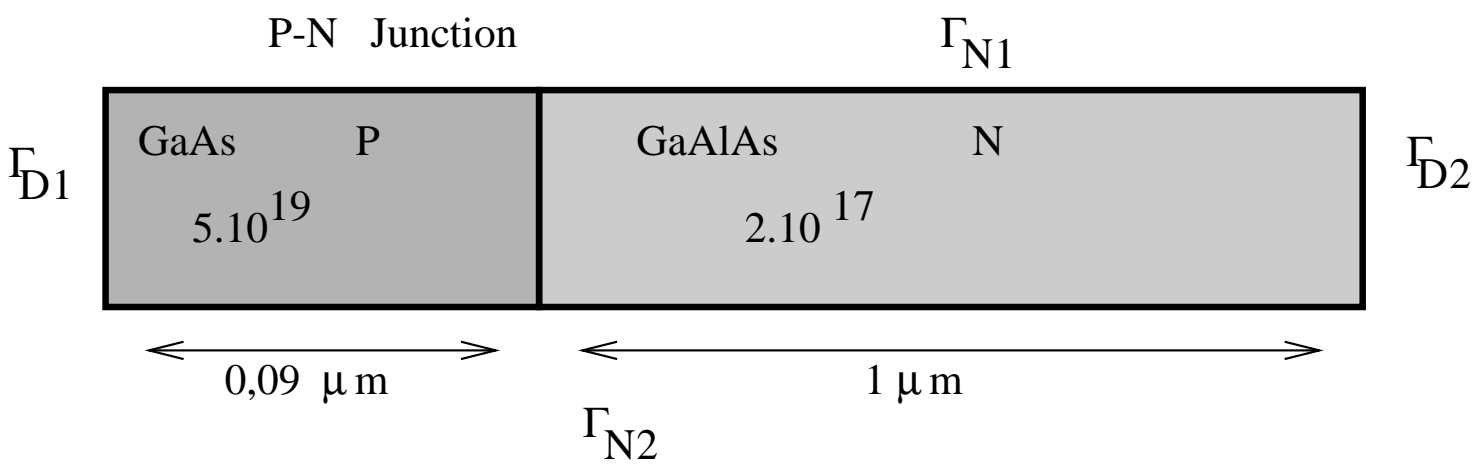

Figure 15: Junction geometry for validation of our numerical results. The parts $\Gamma_{N 1, N 2}$ correspond to the two insulating boundaries (Neumann boundary conitions), and $\Gamma_{D 1, D 2}$ correspond to the two ohmic contacts (Dirichlet boundary conditions).

We consider the (abrupt) heterojunction diode (quasi-1D problem) described by

- Layer 1: length: 1. $\mu m$, Mat: GaAlAs, Type: N, doping $=2.10^{17}$

- Layer 2: length: $0.09 \mu m$, Mat: GaAs, Type: P, doping $=5.10^{19}$

A triangular mesh with size refinements near ohmic contacts and in the neighbouring of the junction is used. Only one layer of triangles is generated for the numerical simulation.

\subsubsection{Description of the solution figures}

The simulation has been carried out for different biases. We compute first the equilibrium state (which is obtained as the solution of the non linear Poisson equation -only-, and corresponds to no applied potential) and then compute the solution of the static problem for different biases by applying successively a voltage increment $\Delta V$ (or loading increment) to the previous state. For each new polarization point, a "prediction" for the solution is calculated by an extension of the procedure given in 
[60]; this predicted solution is used as the initial condition for the time process. By this way relatively large values for the potential increments can be considered. Nevertheless, if $\Delta V$ is too high, numerical difficulties to obtain the new state will appear (-no continuation techniques are presently used for the boundary conditions-).

Let us present on figure (16) -graph : POTENTIAL \& QUASI-F ERMI LEVELS-, the solution of the static problem for $0.25 \mathrm{~V}$ and $1.0 \mathrm{~V}$ forward biases; we can see (continuous thick lines) the electrostatic potential $\phi$ and quasi-Fermi levels $\phi_{n}$ and $\phi_{p}$; only the part around the juntion is represented. Dotted thin lines represent first the conduction and valence band and also the electrostatic potential and quasiFermi levels at previous polarization. The right part of the figure (16) (CARRIER CONCENTRATIONS) presents the electrons and holes distributions at present polarization (continuous thick lines) and also at the previous one (dotted thin lines). The solution for $1 . V$ forward bias was computed using a voltage increment $\Delta V=0.5 V$; if we refer to [32], it seems that mesh sizes at ohmic contacts are not sufficiently thin for such forward bias. Fig. (17) presents the solution for $0.25 \mathrm{~V}$ and $2.0 \mathrm{~V}$ reverse biases. We can notice here that for reverse biases, the numerical problem is more difficult than for forward biases, since the variations of the carrier densities become more strong and abrupt, (see figure (17)). 

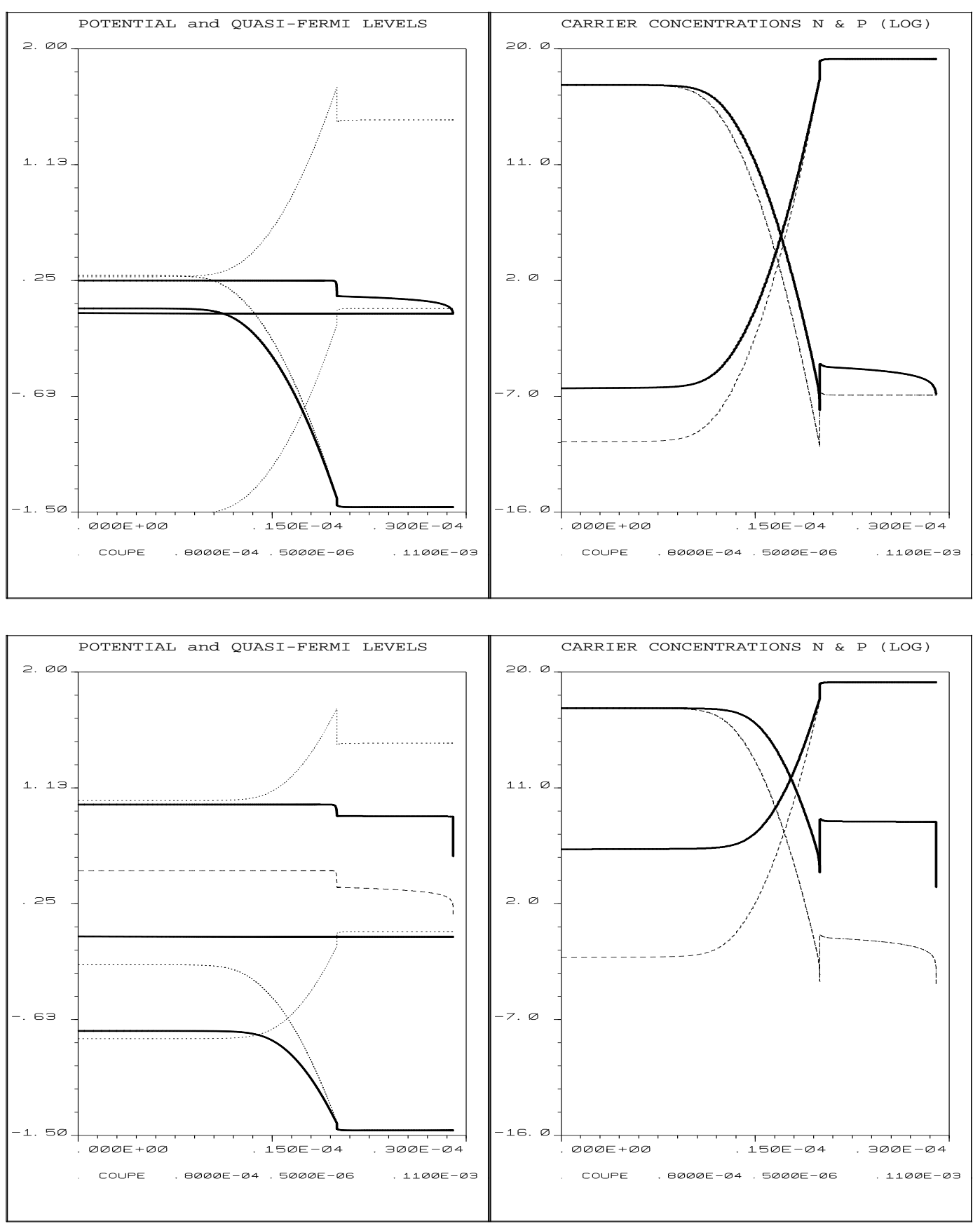

Figure 16: Potential, quasi-Fermi levels, conduction and valence bands, carrier distributions. Graphs at the top: 0.25 V FORWARD BIAS, at the bottom: $1 . \mathrm{V}$ FORWARD BIAS

$\mathrm{RR} \mathrm{n}^{\circ} 2546$ 

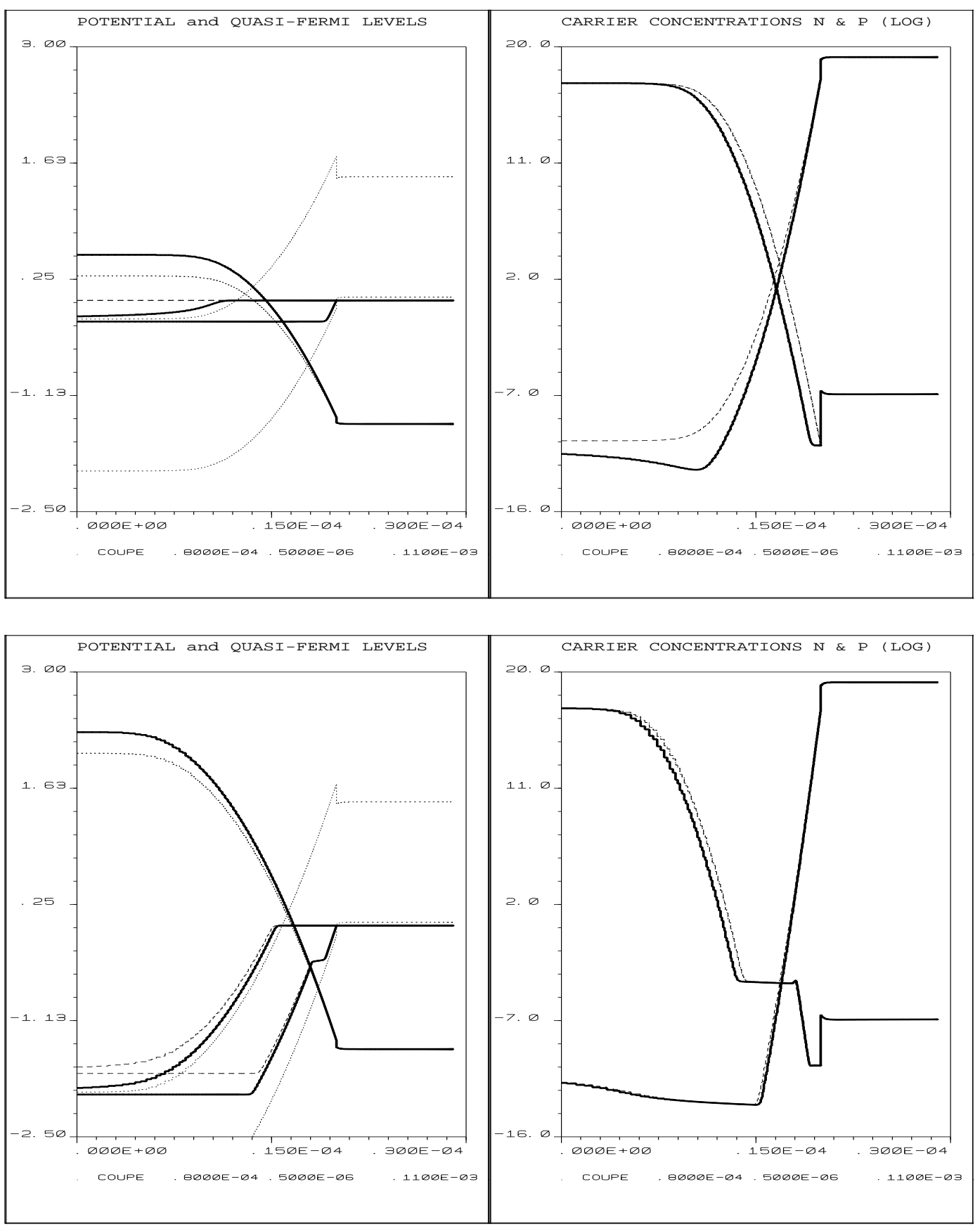

Figure 17: Potential, quasi-Fermi levels, conduction and valence bands, carrier distributions. Graphs at the top: 0.25 V REVERSE BIAS, at the bottom: 2.V REVERSE BIAS 


\subsubsection{Numerical behaviour for algorithm 3 with respect to the voltage increment and the initial time steps}

The following results are for algorithm 3 described in section (4.6). The strategy 2 (113) is used for the increase of the $\Delta s_{i}$ parameters, using (in general)

$$
a=12, \quad b=6
$$

With these parameters, the upper-limit on the factor for the arclength increase is 4 .

The table 1 gives a summary on the convergence behaviour in the forward bias case, with voltage increment $\Delta V=0.25 \mathrm{~V}$. The results are given for different values of the $\lambda_{\text {initial }}$ parameter and for polarizations between 0 . and $2 V$. The number " $N b$ time STEPS" represents the number of continuation steps +1 (the initial step). In the column "CUMUL. Tot. Fact" we give the cumulative total of all matrix factorizations needed to reach the convergence (stationnary solution). In the next three columns we give the number of Newton-Raphson iterations needed for each equation (Eq.1 $\equiv$ Poisson, Eq.2 $\equiv$ electron continuity, Eq.3 $\equiv$ hole continuity) to solve the nonlinear problem for the initial step in the continuation process (with residuals values $\leq 10^{-12}$ ). The last three columns give the maximum number of NewtonRaphson iterations for the correction steps during the continuation procedure. We can remark that we can take relatively large values for the parameter $\bar{\lambda}_{\text {initial }}$ and obtain by this way a very efficient algorithm. Table 2 reflects the algorithm behaviour when the voltage increment is $\Delta V=0.50 \mathrm{~V}$. We cannot see strong differences with the previous table (for $\Delta V=0.25 \mathrm{~V}$ ) except for the number of Newton iterations at initialization step and for the continuity equations. Let us give more details concerning the algorithm behaviour for $\Delta V=0.50 \mathrm{~V}$. We can see on figure (18) results at $0.5 \mathrm{~V}$ forward bias and for $\bar{\lambda}_{\text {initial }}=100, a=12$ and $b=6$. The figure (19) presents the results at $1.5 \mathrm{~V}$ forward bias for the same parameter values.

For all the graphs, abscissa represents the time step (or continuation step) number. On the graph RESIDUALS (LOG SCALE), the first group of curves represents:

- the relative error on the total current conservation: dashed thick line,

If we denote by $I_{C_{i}}$ the total current flowing through the contact $C_{i}$, i.e.

$$
I_{C_{i}}=\int_{C_{i}}\left(\overrightarrow{J_{n}}+\overrightarrow{J_{p}}\right) \cdot \vec{n} d \Gamma
$$

then the relative error is measured by

$$
\frac{\left|\sum_{C_{i}} I_{C_{i}}\right|}{\max \left|I_{C_{i}}\right|}
$$

$\operatorname{RR} n^{\circ} 2546$ 


\begin{tabular}{|c|c|c|c|c|c|c|c|c|c|}
\hline \multicolumn{3}{|c|}{$\begin{array}{l}\text { ALG.3 } \\
\text { Strategy 2: } a=12 \quad b=6\end{array}$} & FORWAR & Bias, & \multicolumn{5}{|c|}{ Tension incr. $\Delta V=0.25 \mathrm{~V}$} \\
\hline \multirow{3}{*}{ Polar. } & \multirow{3}{*}{$\lambda_{i n}$} & \multirow{3}{*}{$\begin{array}{l}\mathrm{Nb} \text { time } \\
\text { STEPS }\end{array}$} & \multirow{3}{*}{$\begin{array}{l}\text { CUMUL. } \\
\text { Tot. Fact }\end{array}$} & \multicolumn{6}{|c|}{ Newton iterations } \\
\hline & & & & \multicolumn{3}{|c|}{ initialisation } & \multicolumn{3}{|c|}{ Cont. steps (Max) } \\
\hline & & & & Eq.1 & Eq.2 & Eq.3 & Eq.1 & Eq.2 & Eq.3 \\
\hline $0.25 \mathrm{~V}$ & 1 & 20 & 231 & 4 & 9 & 4 & 5 & 6 & 8 \\
\hline $0.50 \mathrm{~V}$ & 1 & 20 & 226 & 4 & 7 & 7 & 5 & 6 & 5 \\
\hline $0.75 \mathrm{~V}$ & 1 & 19 & 214 & 4 & 6 & 5 & 5 & 5 & 5 \\
\hline $1.00 \mathrm{~V}$ & 1 & 21 & 228 & 4 & 5 & 4 & 6 & 5 & 5 \\
\hline $1.25 \mathrm{~V}$ & 1 & 24 & 262 & 4 & 5 & 3 & 6 & 5 & 4 \\
\hline $1.50 \mathrm{~V}$ & 1 & 25 & 328 & 6 & 4 & 3 & 6 & 6 & 6 \\
\hline $1.75 \mathrm{~V}$ & 1 & 30 & 360 & 6 & 4 & 4 & 6 & 5 & 5 \\
\hline $2.00 \mathrm{~V}$ & 1 & 24 & 328 & 7 & 4 & 4 & 8 & 6 & 5 \\
\hline $0.25 \mathrm{~V}$ & 100 & 14 & 148 & 6 & 11 & 9 & 5 & 4 & 6 \\
\hline $0.50 \mathrm{~V}$ & 100 & 14 & 149 & 6 & 13 & 8 & 5 & 5 & 5 \\
\hline $0.75 \mathrm{~V}$ & 100 & 14 & 147 & 6 & 7 & 6 & 5 & 5 & 5 \\
\hline $1.00 \mathrm{~V}$ & 100 & 15 & 161 & 6 & 6 & 5 & 5 & 5 & 5 \\
\hline $1.25 \mathrm{~V}$ & 100 & 19 & 202 & 6 & 5 & 4 & 6 & 5 & 4 \\
\hline $1.50 \mathrm{~V}$ & 100 & 20 & 240 & 7 & 5 & 4 & 6 & 5 & 4 \\
\hline $1.75 \mathrm{~V}$ & 100 & 23 & 271 & 8 & 5 & 5 & 6 & 5 & 5 \\
\hline $2.00 \mathrm{~V}$ & 100 & 19 & 258 & 9 & 6 & 4 & 8 & 6 & 4 \\
\hline $0.25 \mathrm{~V}$ & 10000 & 9 & 83 & 6 & 10 & 10 & 4 & 4 & 4 \\
\hline $0.50 \mathrm{~V}$ & 10000 & 9 & 92 & 7 & 15 & 13 & 4 & 4 & 4 \\
\hline $0.75 \mathrm{~V}$ & 10000 & 11 & 106 & 7 & 18 & 8 & 4 & 4 & 4 \\
\hline $1.00 \mathrm{~V}$ & 10000 & 10 & 94 & 7 & 9 & 6 & 4 & 4 & 4 \\
\hline $1.25 \mathrm{~V}$ & 10000 & 14 & 142 & 7 & 6 & 5 & 5 & 5 & 4 \\
\hline $1.50 \mathrm{~V}$ & 10000 & 15 & 177 & 7 & 5 & 4 & 6 & 5 & 4 \\
\hline $1.75 \mathrm{~V}$ & 10000 & 18 & 200 & 8 & 6 & 5 & 6 & 5 & 5 \\
\hline $2.00 \mathrm{~V}$ & 10000 & 14 & 182 & 9 & 10 & 4 & 8 & 7 & 5 \\
\hline
\end{tabular}

Table 1: Summary of results (algorithm behaviour) Alg.3, Forward bias, $\Delta V=$ $0.25 \mathrm{~V}$.

- the $\phi$-residuals: continuous thick line and D-residuals: continuous thin line 


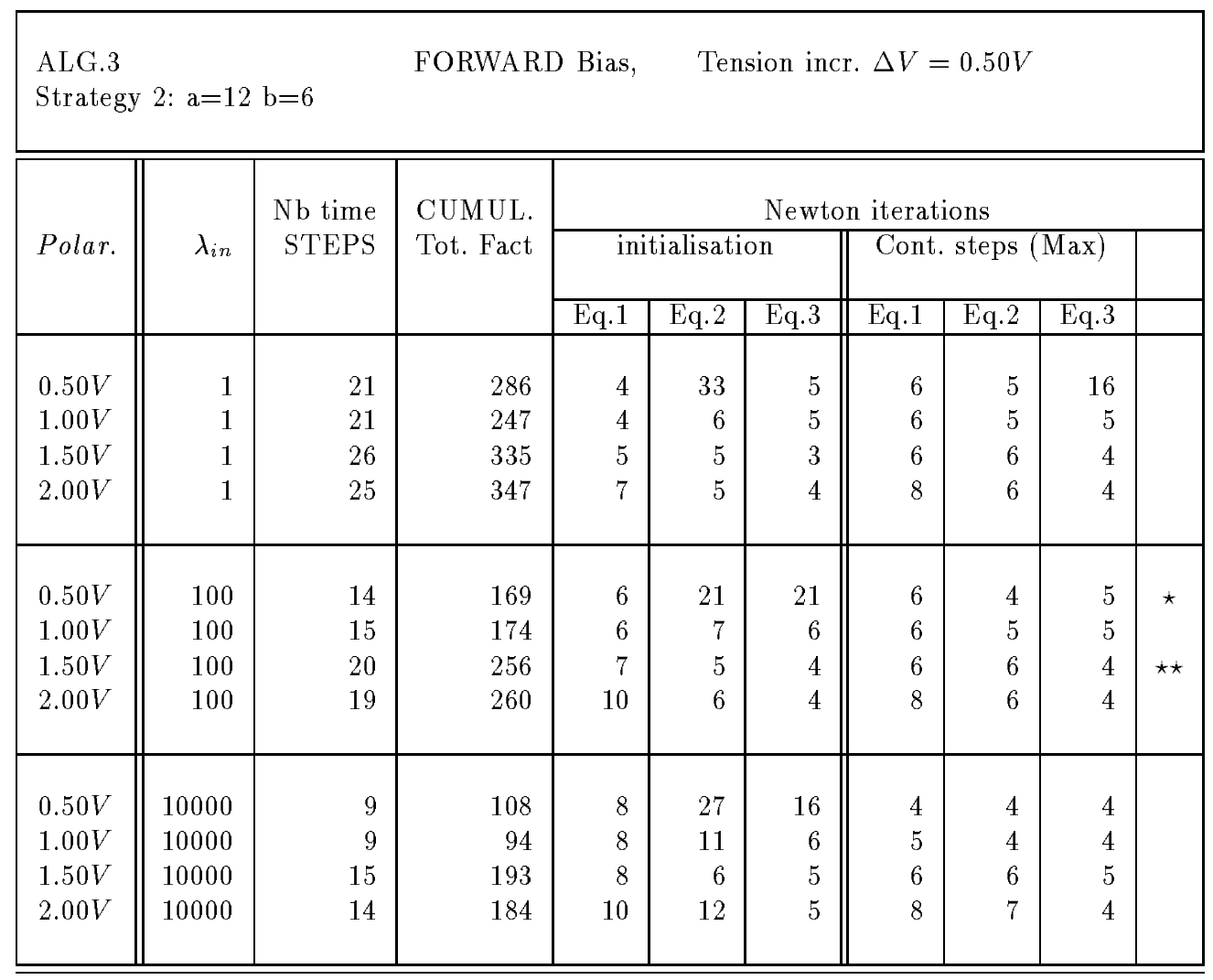

Table 2: Summary of results (algorithm behaviour) Alg.3, Forward bias, $\Delta V=$ $0.50 \mathrm{~V}$.

$\star$ see fig.(18),

$\star \star$ see fig.(19)

- the $\phi_{n}$-residuals: narrow-dotted thick line and $J_{n}$-residuals: narrow-dotted thin line

- the $\phi_{p}$-residuals: wide-dotted thick line and $J_{p}$-residuals: wide-dotted thin line (see algorithm descriptions, detail of $e_{i}$ quantities in (75))

The second group of curves (at the upper part of the graph), maximum variations for the carriers densities are represented (values are shifted around 6 to overcome 
superposition with residuals curves, more precisely

$$
\begin{aligned}
& 6+\left[\operatorname{Max}_{x}\left(\frac{\operatorname{dens}^{k+1}}{\operatorname{dens}^{k}}\right)-1\right] * a m p l \\
& 6+\left[\operatorname{Min}_{x}\left(\frac{\operatorname{dens}^{k+1}}{\operatorname{dens}^{k}}\right)-1\right] * a m p l
\end{aligned}
$$

are drawn. When no variations occur, time step too small or stationary state obtained, these curves tends to the value 6 )

- maximum variations (increase and decrease) for electron density: continuous lines

- maximum variations (increase and decrease) for hole density: dotted lines

On the graph NEWTON ITERATIONS NUMBER, we represent for each time step (or continuation step), the number of Newton steps needed for the correction procedure.

- for the $D$ variable: continuous line

- for the $J_{n}$ variable: narrow-dotted line

- for the $J_{p}$ variable: wide-dotted line

On the graph FACTOR ON LOCAL TIME STEPS (LOG), the curves represent the Log values of the parameter

$$
\bar{\lambda}_{1,2,3}^{k+1}
$$

introduced in $\S 2.4$ (Time discretization). This factor has to be "sufficiently" high in order to be sure that the static solution is obtained.

- factor related to the Poisson equation: continuous line

- factor related to the electron transport equation: narrow-dotted line

- factor related to the hole transport equation: wide-dotted line

On the graph CUMULATIVE TOTAL OF FACTORIZATIONS, we represent the evolution of the cumulative total of factorizations (Cholesky or LU) needed to solve the static problem under consideration (this may be a significant representation of the computational cost). We find again 
- number of Cholesky factorizations related to the Poisson equation: continuous line

- number of LU factorizations related to the electron continuity equation: narrowdotted line

- number of LU factorizations related to the hole continuity equation: widedotted line 


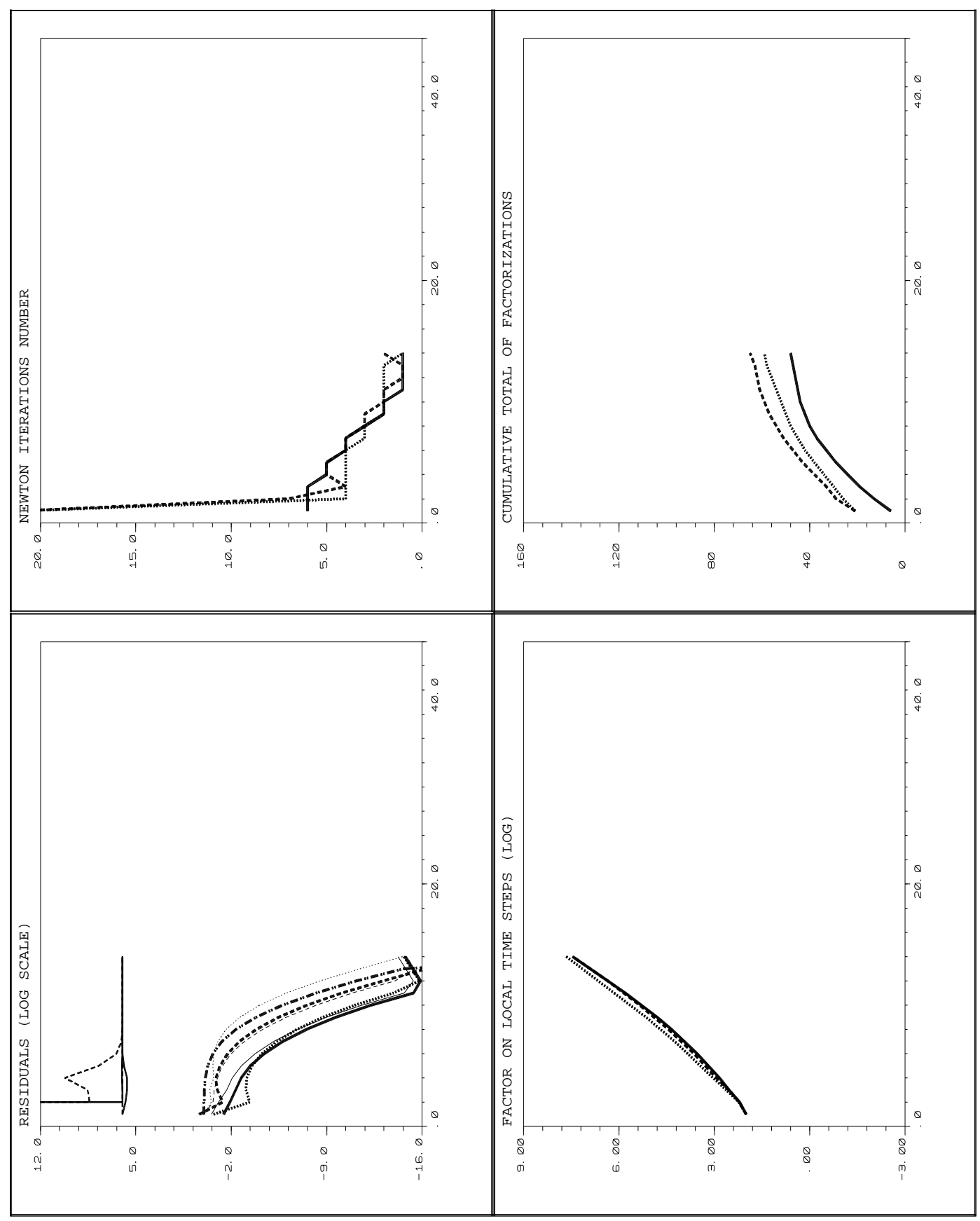

Figure 18: Algorithmic behaviour (Alg:3), ForwardBias : $0.5 \mathrm{~V}, \Delta V=0.5 \mathrm{~V}, \bar{\lambda}_{\text {in }}=$ $100, a=12, b=6$ 

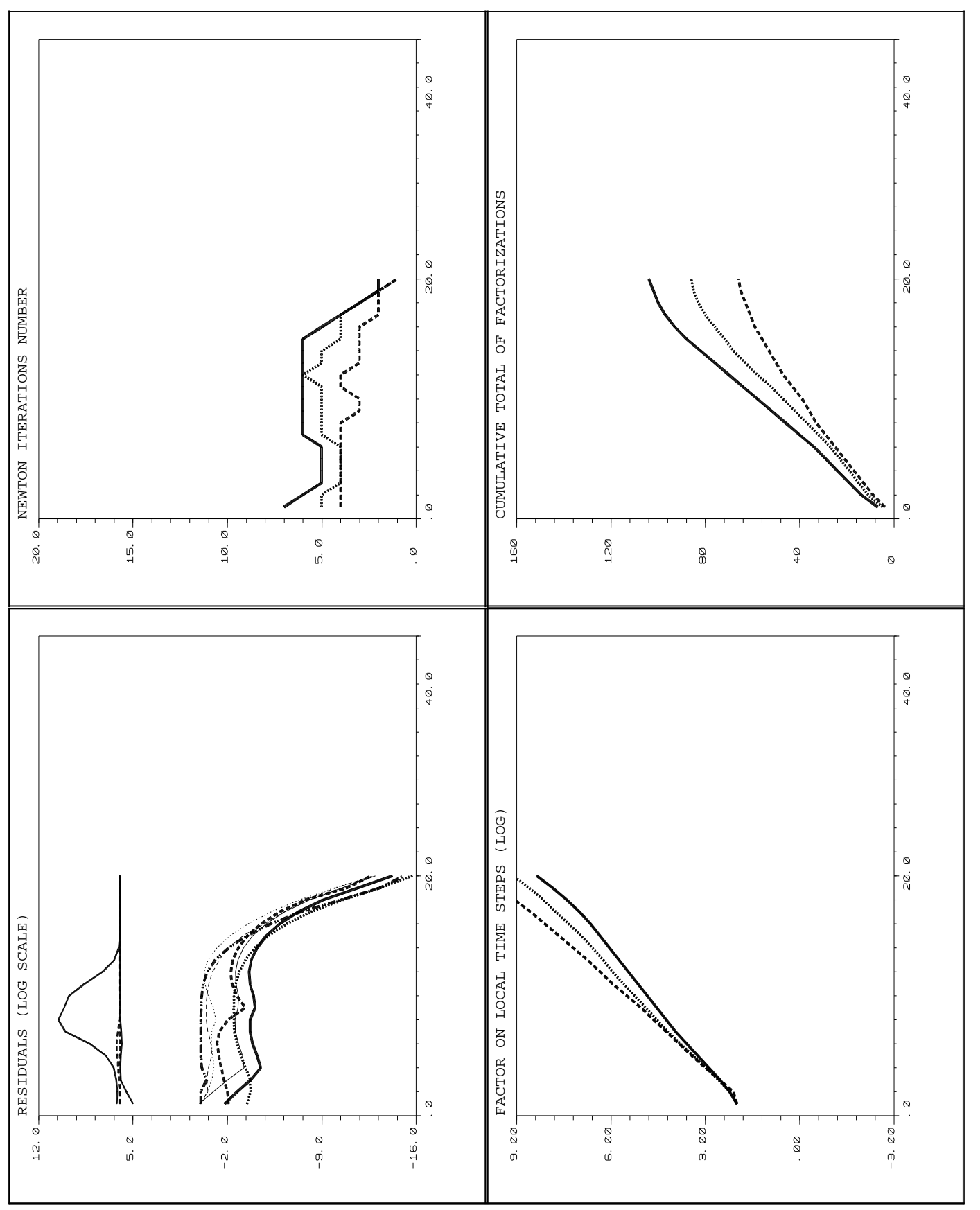

Figure 19: Algorithmic behaviour (Alg:3), ForwardBias : $1.5 \mathrm{~V}, \Delta V=0.5 \mathrm{~V}, \bar{\lambda}_{\text {in }}=$ $100, a=12, b=6$

$\mathrm{RR} \mathrm{n}^{\circ} 2546$ 


\begin{tabular}{|c|c|c|c|c|c|c|c|c|c|}
\hline \multicolumn{3}{|c|}{$\begin{array}{l}\text { ALG.3 } \\
\text { Strategy 2: } a=10 \quad b=3\end{array}$} & \multicolumn{2}{|c|}{ FORWARD Bias, } & \multicolumn{5}{|c|}{ Tension incr. $\Delta V=0.50 \mathrm{~V}$} \\
\hline \multirow{3}{*}{ Polar. } & \multirow{3}{*}{$\lambda_{i n}$} & \multirow{3}{*}{$\begin{array}{l}\mathrm{Nb} \text { time } \\
\text { STEPS }\end{array}$} & \multirow{3}{*}{$\begin{array}{l}\text { CUMUL. } \\
\text { Tot. Fact }\end{array}$} & \multicolumn{6}{|c|}{ Newton iterations } \\
\hline & & & & \multicolumn{3}{|c|}{ initialisation } & \multicolumn{3}{|c|}{ Cont. steps (Max) } \\
\hline & & & & Eq.1 & Eq.2 & Eq.3 & Eq.1 & Eq.2 & Eq.3 \\
\hline $0.50 \mathrm{~V}$ & 1 & 19 & 258 & 4 & 33 & 5 & 6 & 5 & 12 \\
\hline $1.00 \mathrm{~V}$ & 1 & 17 & 208 & 4 & 6 & 5 & 6 & 6 & 6 \\
\hline $1.50 \mathrm{~V}$ & 1 & 21 & 272 & 5 & 5 & 3 & 6 & 6 & 4 \\
\hline $2.00 \mathrm{~V}$ & 1 & 22 & 306 & 7 & 5 & 4 & 8 & 7 & 5 \\
\hline $0.50 \mathrm{~V}$ & 100 & 11 & 153 & 6 & 21 & 21 & 6 & 5 & 5 \\
\hline $1.00 \mathrm{~V}$ & 100 & 13 & 151 & 6 & 7 & 6 & 6 & 6 & 6 \\
\hline $1.50 \mathrm{~V}$ & 100 & 17 & 218 & 7 & 5 & 4 & 6 & 6 & 4 \\
\hline $2.00 \mathrm{~V}$ & 100 & 18 & 245 & 10 & 6 & 4 & 8 & 7 & 5 \\
\hline $0.50 \mathrm{~V}$ & 10000 & 8 & 104 & 8 & 27 & 16 & 4 & 4 & 4 \\
\hline $1.00 \mathrm{~V}$ & 10000 & 8 & 87 & 8 & 11 & 6 & 5 & 4 & 4 \\
\hline $1.50 \mathrm{~V}$ & 10000 & 13 & 172 & 8 & 6 & 5 & 6 & 6 & 5 \\
\hline $2.00 \mathrm{~V}$ & 10000 & 14 & 185 & 10 & 12 & 5 & 8 & 8 & 5 \\
\hline
\end{tabular}

Table 3: Summary of results (algorithm behaviour) Alg.3, Forward bias, $\Delta V=$ $0.50 \mathrm{~V}, \mathrm{a}=10, \mathrm{~b}=3$.

The forward bias case seems to lead to relatively easy problems to solve. We can change $a$ and $b$ in (116) in order to obtain a higher speed in the arclength variations. Table 3 give the results obtained with $a=10$ and $b=3$. We can see that there is some improvement in the cost but not very significant. Variations of parameters $a$ and $b$ in that way may on the other hand favor the possiblities of numerical problems. Larger values than $\Delta V=0.5 \mathrm{~V}$ can be taken as we can see on table 4 , but one has to be more careful with $a, b, \bar{\lambda}_{\text {initial }}$. If $\bar{\lambda}_{\text {initial }}$ is too high, we encounter numerical problems and Newton loop failed at the initialization step and/or during the continuation. If $\lambda_{\text {initial }}$ is too small, similar problems occur if parameters $a$ and $b$ are not suitably adjusted. 


\begin{tabular}{|c|c|c|c|c|c|c|c|c|c|}
\hline \multicolumn{3}{|c|}{$\begin{array}{l}\text { ALG. } 3 \\
\text { Strategy 2: } a=12 b=6\end{array}$} & \multicolumn{2}{|c|}{ FORWARD Bias, } & \multicolumn{5}{|c|}{ Tension incr. $\Delta V=1.00 \mathrm{~V}$} \\
\hline \multirow{3}{*}{ Polar. } & \multirow{3}{*}{$\lambda_{i n}$} & \multirow{3}{*}{$\begin{array}{l}\mathrm{Nb} \text { time } \\
\text { STEPS }\end{array}$} & \multirow{3}{*}{$\begin{array}{l}\text { CUMUL. } \\
\text { Tot. Fact }\end{array}$} & \multicolumn{6}{|c|}{ Newton iterations } \\
\hline & & & & \multicolumn{3}{|c|}{ initialisation } & \multicolumn{3}{|c|}{ Cont. steps (Max) } \\
\hline & & & & Eq.1 & Eq.2 & Eq.3 & Eq.1 & Eq.2 & Eq.3 \\
\hline $1.00 \mathrm{~V}$ & 1 & 57 & 868 & 4 & 10 & 10 & 6 & 24 & 26 \\
\hline $2.00 \mathrm{~V}$ & 1 & 26 & 375 & 7 & 5 & 4 & 7 & 6 & 5 \\
\hline $1.00 \mathrm{~V}$ & 10 & 19 & 248 & 6 & 14 & 17 & 6 & 7 & 8 \\
\hline $2.00 \mathrm{~V}$ & 10 & 23 & 329 & 9 & 6 & 4 & 8 & 6 & 5 \\
\hline $1.00 \mathrm{~V}$ & 100 & 16 & 166 & 7 & 14 & 8 & 7 & 5 & 5 \\
\hline $2.00 \mathrm{~V}$ & 100 & 19 & 272 & 10 & 7 & 5 & 8 & 6 & 5 \\
\hline $1.00 \mathrm{~V}$ & 10000 & $\mathrm{~Pb}$ & $* *$ & $* *$ & $* *$ & $* *$ & $* *$ & $* *$ & $* *$ \\
\hline
\end{tabular}

Table 4: Summary of results (algorithm behaviour) Alg.3, Forward bias, $\Delta V=$ $1.00 \mathrm{~V}$.

Let us now give some numerical results relative to the reverse bias. Table 5 summarize the results for a voltage increment $\Delta V=-0.25 \mathrm{~V}$. The behaviour appears to be strongly similar to the behaviour in the forward bias case for the same voltage increment. It seems however (at present time) that when larger values for $\Delta V$ are taken (-the non linearity and stiffness of the problem under consideration become stronger-), the parameters $\bar{\lambda}_{\text {initial }}, a, b$ are more difficult to choose or adjust. Nevertheless, results can be obtained for example for $\Delta V=-0.40 \mathrm{~V}$ and table 6 presents the algorithmic behaviour obtained ( $a$ and $b$ are choosen in order to have "smoother" increasing factors for the arclengths).

\section{Remarks and comments}




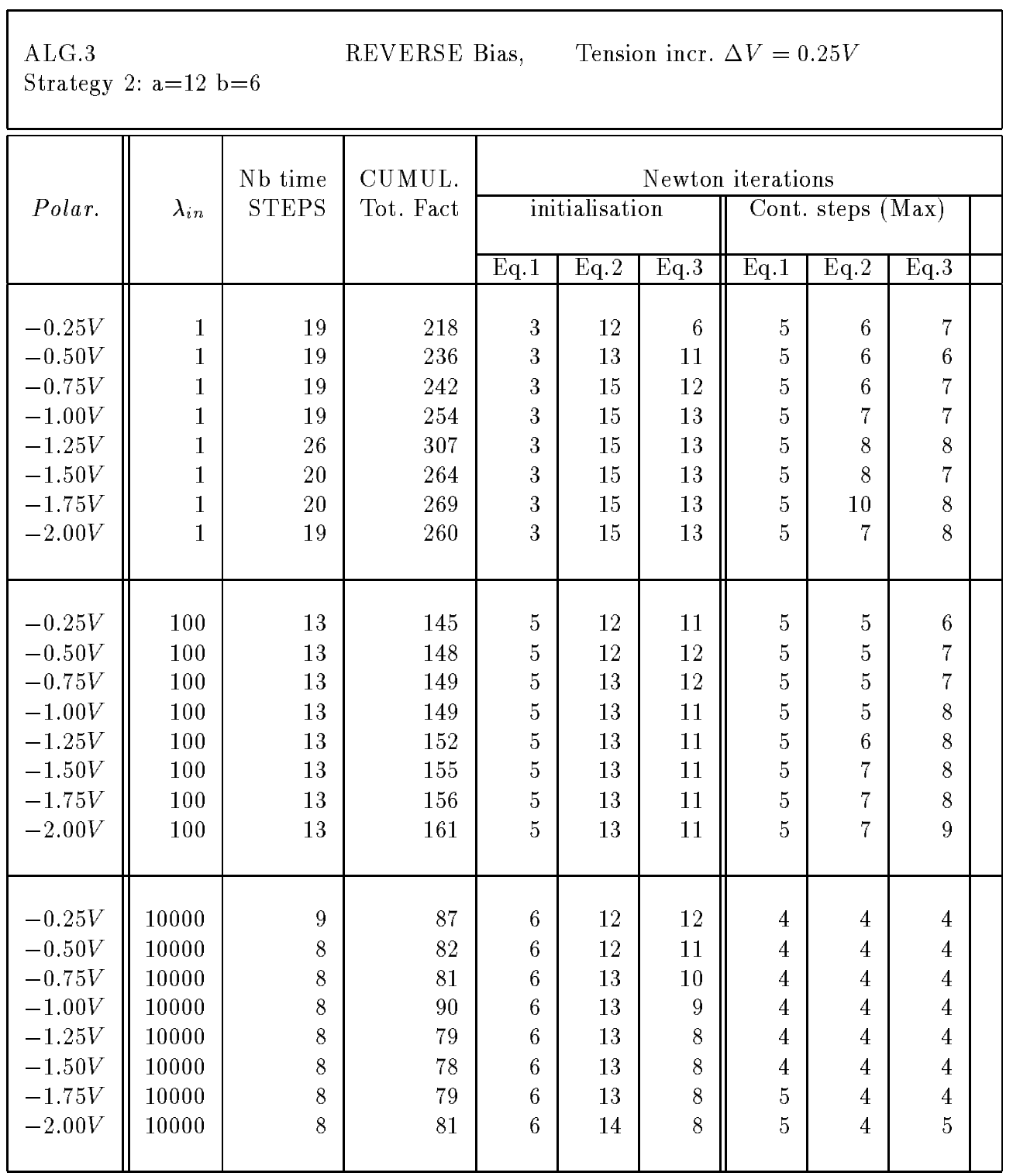

Table 5: Summary of results (algorithm behaviour) Alg.3, Reverse bias, $\Delta V=0.25 \mathrm{~V}$.

- The computations are carried out using extended precision (quad precision on IBM RS6000), and convergence tests $\varepsilon_{1}$ and $\varepsilon_{2}$ (see description of algorithm 2 and 3 ) are taken with the value $10^{-12}$ 


\begin{tabular}{|c|c|c|c|c|c|c|c|c|c|}
\hline \multicolumn{3}{|c|}{$\begin{array}{l}\text { ALG.3 } \\
\text { Strategy } 2: a=15 \quad b=10\end{array}$} & \multicolumn{2}{|c|}{ REVERSE Bias, } & \multicolumn{5}{|c|}{ Tension incr. $\Delta V=0.40 \mathrm{~V}$} \\
\hline \multirow{3}{*}{ Polar. } & \multirow{3}{*}{$\lambda_{i n}$} & \multirow{3}{*}{$\begin{array}{l}\mathrm{Nb} \text { time } \\
\text { STEPS }\end{array}$} & \multirow{3}{*}{$\begin{array}{l}\text { CUMUL. } \\
\text { Tot. Fact }\end{array}$} & \multicolumn{6}{|c|}{ Newton iterations } \\
\hline & & & & \multicolumn{3}{|c|}{ initialisation } & \multicolumn{3}{|c|}{ Cont. steps (Max) } \\
\hline & & & & Eq.1 & Eq.2 & Eq.3 & Eq.1 & Eq.2 & Eq.3 \\
\hline$-0.40 \mathrm{~V}$ & 100 & 15 & 166 & 6 & 13 & 13 & 5 & 5 & 7 \\
\hline$-0.80 \mathrm{~V}$ & 100 & 42 & 427 & 6 & 21 & 13 & 5 & 39 & 13 \\
\hline$-1.20 \mathrm{~V}$ & 100 & 69 & 678 & 5 & 22 & 12 & 5 & 24 & 11 \\
\hline$-1.60 \mathrm{~V}$ & 100 & 223 & 2414 & 5 & 21 & 12 & 5 & 26 & 13 \\
\hline$-2.00 \mathrm{~V}$ & 100 & 85 & 774 & 5 & 21 & 12 & 5 & 21 & 14 \\
\hline$-2.40 \mathrm{~V}$ & 100 & 109 & 960 & 5 & 21 & 12 & 5 & 27 & 15 \\
\hline$-2.80 \mathrm{~V}$ & 100 & 190 & 2150 & 5 & 21 & 12 & 5 & 29 & 16 \\
\hline$-3.20 \mathrm{~V}$ & 100 & 114 & 1004 & 5 & 21 & 12 & 5 & 24 & 17 \\
\hline
\end{tabular}

Table 6: Summary of results (algorithm behaviour) Alg.3, Reverse bias, $\Delta V=0.40 \mathrm{~V}$.

- It appears from previous results that for not too large voltage increment $\Delta V \sim$ $0.25 \mathrm{~V}$, "large time steps" 100,1000 can be chosen, the static solution for a new bias point is obtained in any case with less than 20 (continuation) time steps.

- If $\bar{\lambda}_{\text {initial }}$ is too small, for example taking the value 0.01 , some numerical troubles may appear. At $+0.5 \mathrm{~V}$ bias value (with $\Delta V=0.5 \mathrm{~V}$ ) and with $a=12$ and $b=6$ as in the most of previous numerical experimentations, no practical convergence has been obtained. In fact, this problem may be related with the choice of the parameters $a$ and $b$ in the strategy 2 for increasing $\Delta s_{i}$. This strategy leads to "too high factor". The reason is certainly that in this context, the number of Newton iterations $n$ needed in corrector step being small with respect to the expected value $a$, the increasing factor for the time steps becomes to high. Then, it seems reasonnable that when we start with "small time steps", the expected value $a$ has to decrease. In fact, taking $a=9$, a convergence (even if it remains laborious) is however obtained.

- For reasonable voltage increment $\Delta V$, the choice for the initial factor $\bar{\lambda}_{\text {initial }}$ on the local time steps, do not seems to be very crucial. 
- Boundary layer modelization for the quasi-Fermi levels [32] can be used for the description and approximation of the static problem (functions $H_{21, i}$ and $H_{31, i}$ given by (25) (27) have to be slightly changed). Global algorithmic behaviour of the continuation techniques remain the same.

- In the reverse bias mode, the algorithm behaviour remains the same, for example, with a voltage increment $\Delta V=0.25 \mathrm{~V}$ (with parameters $\bar{\lambda}_{\text {initial }}=$ $100, a=12, b=6)$, less than 15 continuation steps are needed for each polarization point (as in forward case), for a comparable computational cost. It seems however (at present time) that when larger values for $\Delta V$ are taken (-the nonlinearity of the problem under consideration becomes stronger-), the differents parameters $\bar{\lambda}_{\text {initial }}, a, b$, are more difficult to choose. Certainly control on the curvature as mentionned in $\S 5$ would improve algorithm efficiency in this case.

On table 7 (forward bias) and table 8 (reverse bias) are reported extrema values of carrier densities variations during Newton loops (index $n$ ), i.e. for electrons

$$
\begin{array}{ll}
\max _{k, n} \frac{N^{k+1, n+1}}{N^{k}} & \text { (increase) } \\
\min _{k, n} \frac{N^{k+1, n+1}}{N^{k}} & \text { (decrease) }
\end{array}
$$

and variations with respect to the artificial transient (index $k$ only)

$$
\begin{array}{ll}
\max _{k} \frac{N^{k+1}}{N^{k}} & \text { (increase) } \\
\min _{k} \frac{N^{k+1}}{N^{k}} & \text { (decrease) }
\end{array}
$$

When too strong variations on densities are detected, the factor $\bar{\lambda}_{j}^{k+1}$ is decreased until acceptable variations occur (prescribed values). For example, in reverse bias case with voltage increment $\Delta V=0.4 V$ (see table 8 ) high variations on electrons densities occur at the initialization step and the corresponding value of $\lambda_{\text {in }}$ is then reduced from 100 to $10^{-5}$ or $10^{-6}$ in order to obtain acceptable values for these variations.

If we compare variations with respect to time steps, we can see that there are much higher in reverse bias for $\Delta V=0.4 V$ than for forward bias with $\Delta V=0.5 V$. Reverse bias problems are more stiff than forward bias ones. 


\begin{tabular}{|c|c|c|c|c|c|}
\hline \multicolumn{2}{|c|}{ ALG.3 } & \multicolumn{4}{|l|}{ ORWARD Bias } \\
\hline \multirow[t]{2}{*}{$\lambda_{i n}$} & \multicolumn{2}{|c|}{$\begin{array}{l}\text { Max. densities variations } \\
\text { during NEWTON iterations }\end{array}$} & \multicolumn{2}{|c|}{$\begin{array}{l}\text { Max. densities variations } \\
\text { relatively to time steps }\end{array}$} & \\
\hline & DECREASE & INCREASE & DECREASE & INCREASE & \\
\hline \multicolumn{6}{|c|}{$\Delta V=0.25 \mathrm{~V}$} \\
\hline $\begin{array}{c}1 \\
100 \\
10^{4}\end{array}$ & $\begin{array}{r}3.910^{-1} \rightarrow 3.910^{-1} \\
6.910^{-1} \searrow 5.010^{-1} \\
9.910^{-1} \searrow 7.910^{-28}\end{array}$ & $\begin{array}{c}8.510^{+1} \rightarrow 8.510^{+1} \\
1.810^{+3} \nearrow 1.010^{+4} \\
3.910^{+3} \nearrow 2.610^{+19}\end{array}$ & $\begin{array}{l}3.910^{-1} \rightarrow 3.910^{-1} \\
6.910^{-1} \rightarrow 6.910^{-1} \\
9.910^{-1} \searrow 3.610^{-1}\end{array}$ & $\begin{array}{l}4.710^{+1} \rightarrow 4.710^{+1} \\
8.910^{+1} \nearrow 4.910^{+2} \\
1.010^{+2} \nearrow 2.710^{+3}\end{array}$ & $\begin{array}{l}\mathrm{N} \\
\mathrm{N} \\
\mathrm{N}\end{array}$ \\
\hline $\begin{array}{c}1 \\
100 \\
10^{4}\end{array}$ & $\begin{array}{l}3.410^{-1} \rightarrow 3.410^{-1} \\
2.810^{-1} \rightarrow 2.810^{-1} \\
9.610^{-1} \searrow 4.910^{-2}\end{array}$ & $\begin{array}{r}9.9 \nearrow 3.110^{+1} \\
3.610^{+1} \nearrow 8.610^{+2} \\
2.910^{+3} \nearrow 4.110^{+4}\end{array}$ & $\begin{array}{l}3.410^{-1} \rightarrow 3.410^{-1} \\
2.810^{-1} \rightarrow 2.810^{-1} \\
9.610^{-1} \searrow 8.110^{-1}\end{array}$ & $\begin{array}{r}6.6 \nearrow 1.810^{+1} \\
2.210^{+1} \nearrow 2.310^{+2} \\
1.810^{+2} \nearrow 1.210^{+3}\end{array}$ & $\begin{array}{l}\mathrm{P} \\
\mathrm{P} \\
\mathrm{P}\end{array}$ \\
\hline \multicolumn{6}{|c|}{$\Delta V=0.50 \mathrm{~V}$} \\
\hline $\begin{array}{c}1 \\
100 \\
10^{4}\end{array}$ & $\begin{array}{l}1.710^{-5} \rightarrow 1.710^{-5} \\
4.910^{-1} \rightarrow 4.910^{-1} \\
7.310^{-8} \rightarrow 7.310^{-8}\end{array}$ & $\begin{array}{r}5.410^{+12} \rightarrow 5.410^{+12} \\
2.210^{+8} \rightarrow 2.210^{+8} \\
2.210^{+8} \rightarrow 2.210^{+8}\end{array}$ & $\begin{array}{l}2.210^{-1} \rightarrow 2.210^{-1} \\
4.910^{-1} \rightarrow 4.910^{-1} \\
9.910^{-1} \searrow 2.510^{-1}\end{array}$ & $\begin{array}{l}3.910^{+5} \rightarrow 3.910^{+5} \\
5.610^{+5} \rightarrow 5.610^{+5} \\
4.710^{+5} \rightarrow 4.710^{+5}\end{array}$ & $\begin{array}{l}\mathrm{N} \\
\mathrm{N} \\
\mathrm{N}\end{array}$ \\
\hline $\begin{array}{c}1 \\
100 \\
10^{4}\end{array}$ & $\begin{array}{l}2.410^{-1} \rightarrow 2.410^{-1} \\
9.510^{-2} \rightarrow 9.510^{-2} \\
9.310^{-1} \searrow 7.410^{-1}\end{array}$ & $\begin{array}{l}1.410^{+1} \rightarrow 1.410^{+1} \\
3.910^{+7} \rightarrow 3.910^{+7} \\
1.110^{+8} \rightarrow 1.110^{+8}\end{array}$ & $\begin{array}{l}2.410^{-1} \rightarrow 2.410^{-1} \\
9.510^{-2} \rightarrow 9.510^{-2} \\
9.310^{-1} \searrow 7.410^{-1}\end{array}$ & $\begin{array}{r}5.9 \rightarrow 5.9 \\
3.110^{+5} \rightarrow 3.110^{+5} \\
1.010^{+6} \rightarrow 1.010^{+6}\end{array}$ & $\begin{array}{l}\mathrm{P} \\
\mathrm{P} \\
\mathrm{P}\end{array}$ \\
\hline \multicolumn{6}{|c|}{$\Delta V=1.00 \mathrm{~V}$} \\
\hline $\begin{array}{c}1 \\
100 \\
10^{4}\end{array}$ & $\begin{array}{r}6.010^{-27} \rightarrow 6.010^{-27} \\
3.410^{-1} \rightarrow 3.410^{-1} \\
5.810^{-1}\end{array}$ & $\begin{array}{r}1.810^{+25} \rightarrow 1.8410^{+25} \\
5.110^{+16} \rightarrow 5.110^{+16} \\
5.410^{+16}\end{array}$ & $\begin{aligned} 7.810^{-2} \rightarrow & 7.810^{-2} \\
3.410^{-1} \rightarrow & 3.410^{-1} \\
& 9.910^{-1}\end{aligned}$ & $\begin{array}{r}8.910^{+14} \rightarrow 8.910^{+14} \\
2.610^{+15} \rightarrow 2.610^{+15} \\
1.710^{+15}\end{array}$ & $\begin{array}{l}\mathrm{N} \\
\mathrm{N} \\
*\end{array}$ \\
\hline $\begin{array}{c}1 \\
100 \\
10^{4}\end{array}$ & $\begin{array}{r}7.210^{-5} \rightarrow 7.210^{-5} \\
5.010^{-2} \rightarrow 5.010^{-2} \\
8.810^{-33}\end{array}$ & $\begin{aligned} 1.910^{+10} \rightarrow & 1.910^{+10} \\
1.610^{+16} \rightarrow & 1.610^{+16} \\
& 2.510^{+20}\end{aligned}$ & $\begin{aligned} 4.310^{-2} \rightarrow & 4.310^{-2} \\
5.010^{-2} \rightarrow & 5.010^{-2} \\
& 3.710^{-5}\end{aligned}$ & $\begin{array}{r}1.910^{+4} \rightarrow 1.910^{+4} \\
1.310^{+16} \rightarrow 1.310^{+16} \\
2.510^{+16}\end{array}$ & $\begin{array}{l}\mathrm{P} \\
\mathrm{P} \\
*\end{array}$ \\
\hline
\end{tabular}

Table 7: Carrier densities variations. Forward bias.

* Oscillations, no convergence obtained after 100 time steps.

\subsubsection{Numerical behaviour for algorithm 2}

We consider only one example: we compute the static states for the forward bias points using a voltage increment $\Delta V=0.5, \bar{\lambda}_{\text {initial }}=100, a=12$ and $b=6$ (table 


\begin{tabular}{|c|c|c|c|c|c|}
\hline \multicolumn{2}{|c|}{ ALG.3 } & \multicolumn{4}{|l|}{ REVERSE Bias } \\
\hline \multirow[t]{2}{*}{$\lambda_{i n}$} & \multicolumn{2}{|c|}{$\begin{array}{l}\text { Max. densities variations } \\
\text { during NEWTON iterations }\end{array}$} & \multicolumn{2}{|c|}{$\begin{array}{l}\text { Max. densities variations } \\
\text { relatively to time steps }\end{array}$} & \\
\hline & DECREASE & INCREASE & DECREASE & INCREASE & \\
\hline \multicolumn{6}{|c|}{$\Delta V=0.25 \mathrm{~V}$} \\
\hline $\begin{array}{c}1 \\
100 \\
10^{4}\end{array}$ & $\begin{array}{l}2.410^{-1} \searrow 4.210^{-2} \\
2.610^{-1} \searrow 2.110^{-1} \\
9.410^{-1} \searrow 7.910^{-1}\end{array}$ & $\begin{array}{l}8.710^{+1} \nearrow 1.510^{+4} \\
1.710^{+2} \nearrow 3.110^{+4} \\
1.710^{+2} \nearrow 1.410^{+4}\end{array}$ & $\begin{array}{l}2.410^{-1} \rightarrow 2.410^{-1} \\
2.610^{-1} \searrow 2.110^{-1} \\
9.410^{-1} \searrow 8.810^{-1}\end{array}$ & $\begin{array}{l}8.710^{+1} \text { ノ } 1.510^{+4} \\
1.710^{+2} \nearrow 1.310^{+4} \\
1.710^{+2} \nearrow 1.410^{+4}\end{array}$ & $\begin{array}{l}\mathrm{N} \\
\mathrm{N} \\
\mathrm{N}\end{array}$ \\
\hline $\begin{array}{c}1 \\
100 \\
10^{4}\end{array}$ & $\begin{array}{r}2.610^{-1} \searrow 6.510^{-13} \\
6.910^{-1} \searrow 1.210^{-1} \\
9.910^{-1} \searrow 2.710^{-2}\end{array}$ & $\begin{array}{r}4.7 \nearrow 1.310^{+34} \\
3.410^{+1} \nearrow 4.110^{+2} \\
1.710^{+2} \rightarrow 1.710^{+2}\end{array}$ & $\begin{array}{l}2.610^{-1} \rightarrow 2.610^{-1} \\
6.910^{-1} \searrow 6.410^{-1} \\
9.910^{-1} \searrow 2.010^{-1}\end{array}$ & $\begin{array}{r}4.2 \nearrow 4.510^{+2} \\
3.410^{+1} \nearrow 4.110^{+2} \\
1.710^{+2} \rightarrow 1.710^{+2}\end{array}$ & $\begin{array}{l}\mathrm{P} \\
\mathrm{P} \\
\mathrm{P}\end{array}$ \\
\hline \multicolumn{6}{|c|}{$\Delta V=0.40 \mathrm{~V}$} \\
\hline 100 & $1.110^{-1} \searrow 5.310^{-20}$ & $1.110^{+3} / 6.810^{+34}$ & $1.110^{-1} \searrow 2.610^{-6}$ & $1.110^{+3} \nearrow 2.110^{+9}$ & $\mathrm{~N}$ \\
\hline 100 & $5.510^{-1} \searrow 3.710^{-5}$ & $2.310^{+2} \nearrow 1.710^{+3}$ & $5.510^{-1} \searrow 4.910^{-1}$ & $2.310^{+2} \nearrow 1.710^{+3}$ & $\mathrm{P}$ \\
\hline
\end{tabular}

Table 8: Carrier densities variations. Reverse bias.

9). We present on fig.( 20,21$)$ the numerical behaviour of Algorithm 2, (described in detail in $\S 4.1)$. See fig. $(18,19)$ and table (2) for the results obtained with Algorithm 3 in the same conditions. It appears clearly that the number of iterations needed in the Newton correction step is slightly higher for Algorithm 2 than for Algorithm 3, as expected. 


\begin{tabular}{|c|c|c|c|c|c|c|c|c|c|c|}
\hline \multicolumn{3}{|c|}{$\begin{array}{l}\text { ALG.2 } \\
\text { Strategy } 2: a=12 b=6\end{array}$} & FORWAR & Bias, & \multicolumn{6}{|c|}{ Tension incr. $\Delta V=0.50 \mathrm{~V}$} \\
\hline \multirow{3}{*}{ Polar. } & \multirow{3}{*}{$\lambda_{i n}$} & \multirow{3}{*}{$\begin{array}{l}\mathrm{Nb} \text { time } \\
\text { STEPS }\end{array}$} & \multirow{3}{*}{$\begin{array}{l}\text { CUMUL. } \\
\text { Tot. Fact }\end{array}$} & \multicolumn{7}{|c|}{ Newton iterations } \\
\hline & & & & \multicolumn{3}{|c|}{ initialisation } & \multicolumn{3}{|c|}{ Cont. steps (Max) } & \\
\hline & & & & Eq.1 & Eq.2 & Eq.3 & Eq.1 & Eq.2 & Eq.3 & \\
\hline $0.50 \mathrm{~V}$ & 1 & 45 & 1544 & 4 & 33 & 5 & 14 & 72 & 72 & \\
\hline $1.00 \mathrm{~V}$ & 1 & 22 & 357 & 4 & 6 & 5 & 14 & 7 & 11 & \\
\hline $1.50 \mathrm{~V}$ & 1 & 28 & 536 & 5 & 5 & 3 & 14 & 15 & 10 & \\
\hline $2.00 \mathrm{~V}$ & 1 & 27 & 573 & 7 & 5 & 4 & 15 & 11 & 13 & \\
\hline $0.50 \mathrm{~V}$ & 100 & 15 & 238 & 6 & 21 & 21 & 13 & 10 & 9 & $*$ \\
\hline $1.00 \mathrm{~V}$ & 100 & 16 & 234 & 6 & 7 & 6 & 12 & 9 & 9 & \\
\hline $1.50 \mathrm{~V}$ & 100 & 28 & 371 & 7 & 5 & 4 & 8 & 10 & 14 & $* *$ \\
\hline $2.00 \mathrm{~V}$ & 100 & 27 & 388 & 10 & 6 & 4 & 7 & 13 & 12 & \\
\hline $0.50 \mathrm{~V}$ & 10000 & 9 & 140 & 8 & 27 & 16 & 4 & 10 & 5 & \\
\hline $1.00 \mathrm{~V}$ & 10000 & 9 & 129 & 8 & 11 & 6 & 5 & 9 & 5 & \\
\hline $1.50 \mathrm{~V}$ & 10000 & 15 & 263 & 8 & 6 & 5 & 6 & 9 & 12 & \\
\hline $2.00 \mathrm{~V}$ & 10000 & 14 & 248 & 10 & 12 & 5 & 7 & 17 & 15 & \\
\hline
\end{tabular}

Table 9: Summary of results (algorithm behaviour) Alg.2, Forward bias, $\Delta V=$ $0.50 \mathrm{~V}$.

$\star$ see fig.(20),

$\star \star$ see fig.(21) 


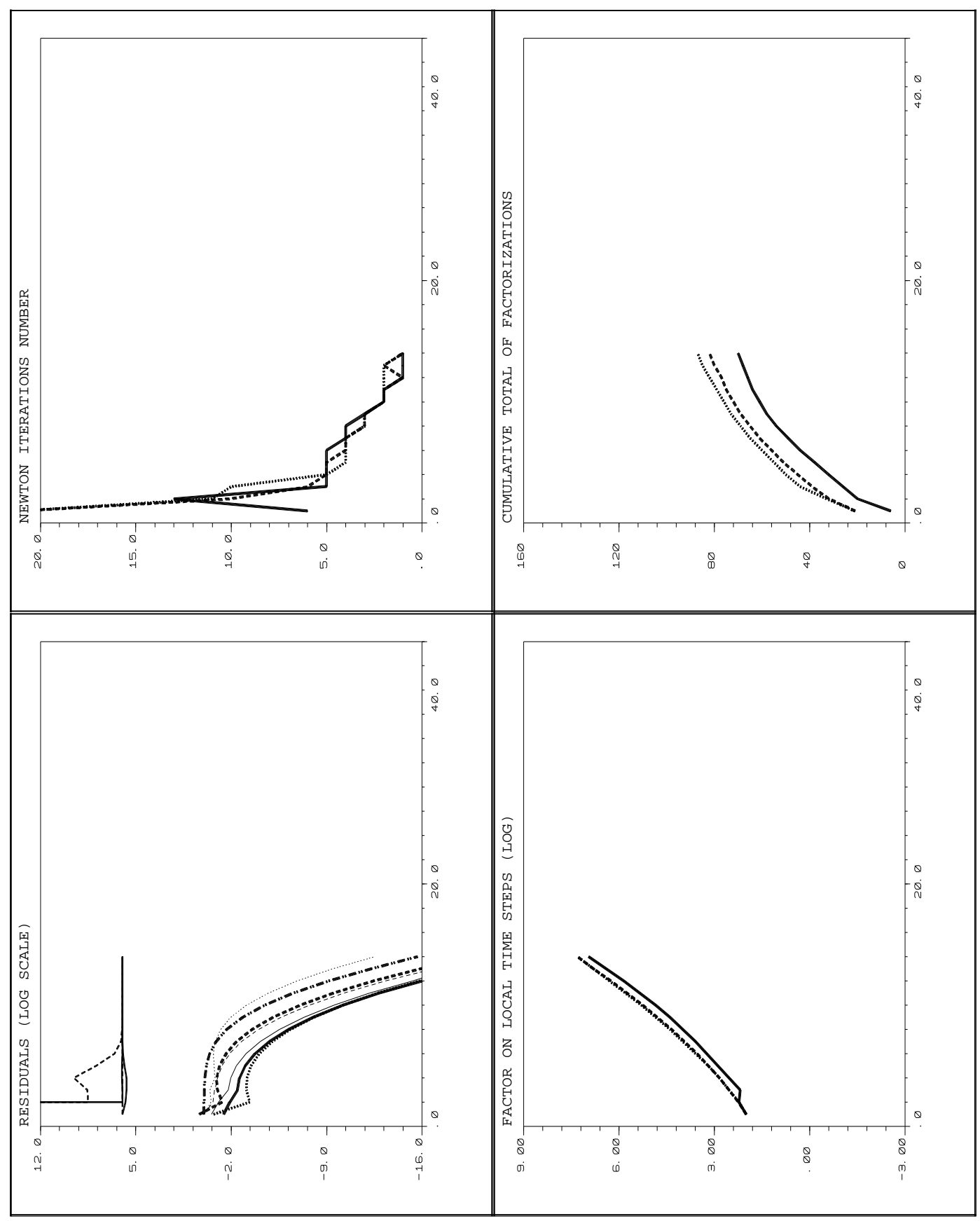

Figure 20: Algorithmic behaviour (Alg:2), ForwardBias : $0.5 \mathrm{~V}, \Delta V=0.5 \mathrm{~V}, \bar{\lambda}_{\text {in }}=$ $100, a=12, b=6$ 


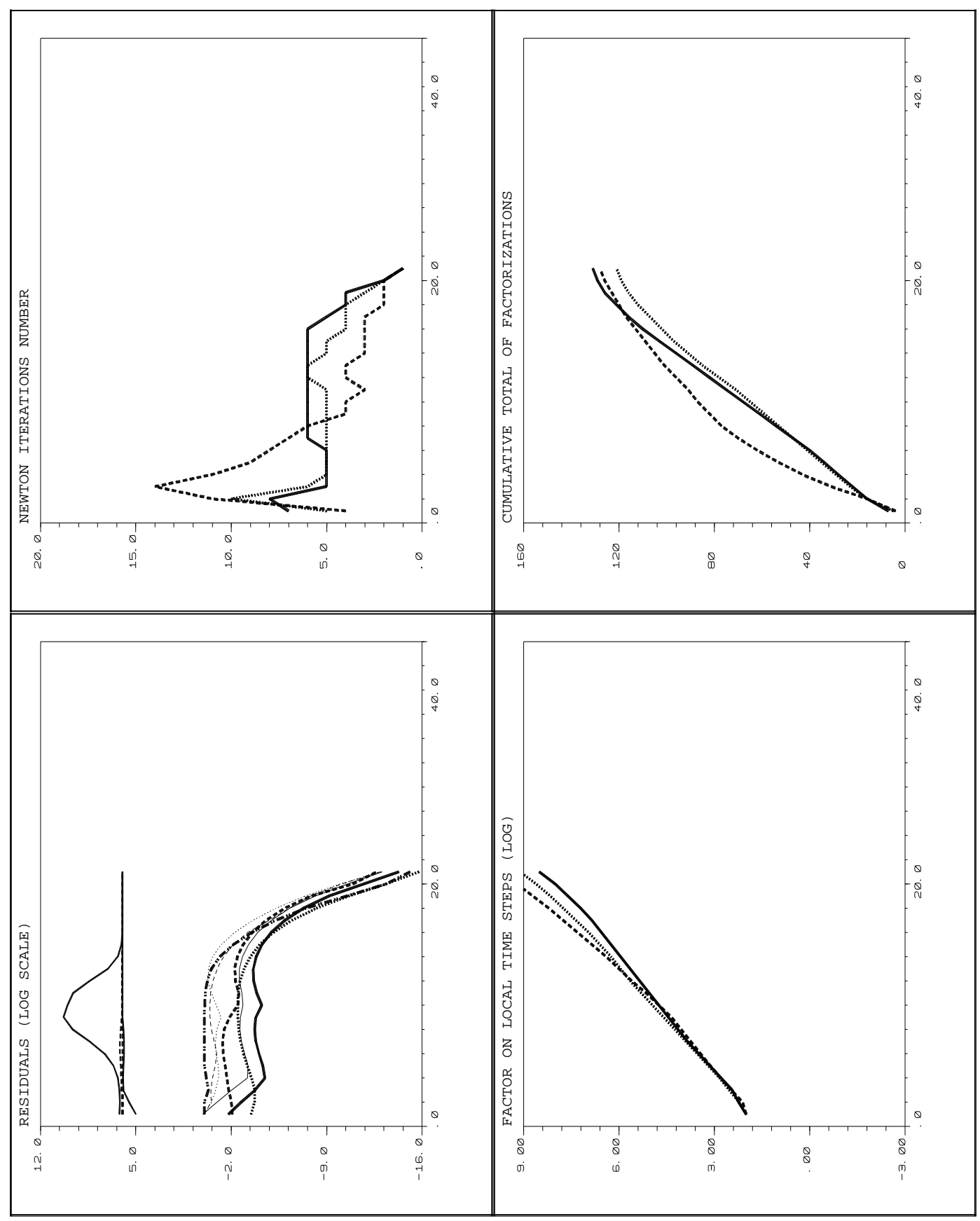

Figure 21: Algorithmic behaviour (Alg:2), ForwardBias : $1.5 \mathrm{~V}, \Delta V=0.5 \mathrm{~V}, \bar{\lambda}_{\text {in }}=$ $100, a=12, b=6$

$\mathrm{RR} \mathrm{n}^{\circ} 2546$ 


\subsection{Heterojunction Bipolar Transistor (H.B.T.)}

We consider a "model" H.B.T. of which a physical description is given by fig.(22).

- Emitter, Mat: GaAlAs, Type: $N^{+}$, doping $=2.10^{17}$

- Base, Mat: GaAs, Type: $P^{++}$, doping $=5.10^{18}$

- Collector, Mat: GaAs, Type: N, doping $=5.10^{16}$

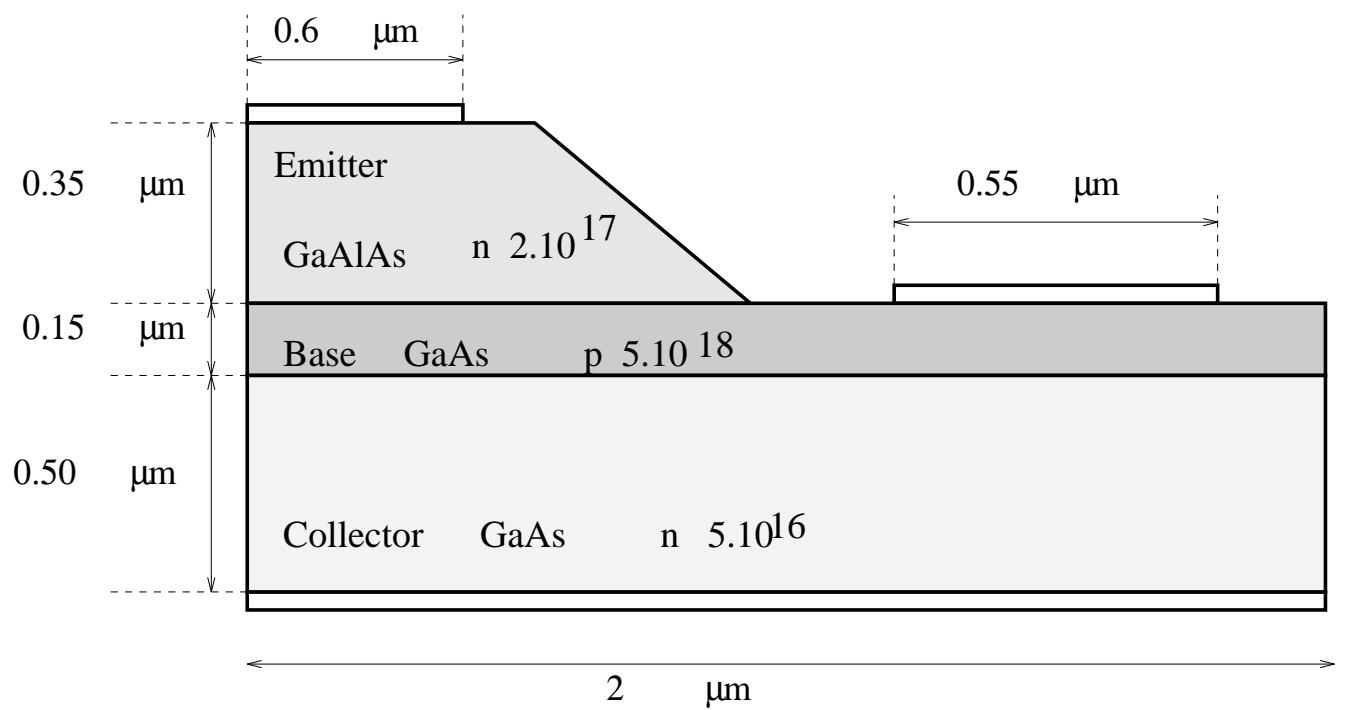

Figure 22: Description of the heterojunction bipolar transistor structure.

All the junctions are considered to be abrupt. For the numerical tests, the applied potential to the collector ohmic contact will remain to $0 . V$. We are presently interested to compute the solution of the static problem for

$$
V_{B} \in[-1,0]
$$

with

$$
V_{C E}=V_{C}-V_{E}=1.4 \mathrm{~V}
$$

(we are in the so-called normal-direct regime, the (HBT) is opertating in common emitter)

The successive static states are computed using the following path 


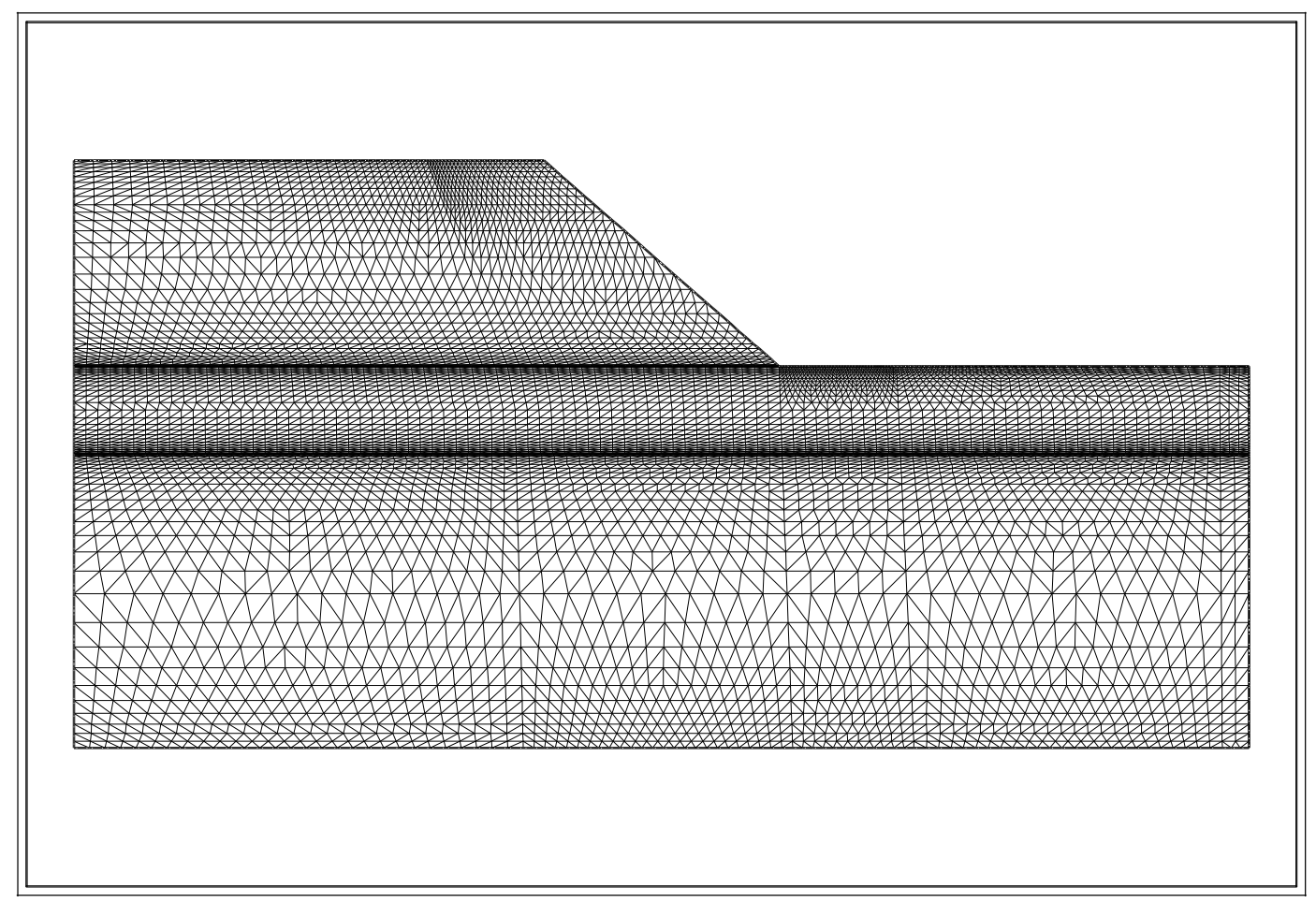

Figure 23: Triangular mesh on the H.B.T.

$\begin{array}{llll}V_{C}=0 . V & V_{B}=0 . V & V_{E}=0 . V & \text { (Equilibrium state) } \\ V_{C}=0 . V & V_{B}=0 . V & V_{E}=-0.2 V & \\ V_{C}=0 . V & V_{B}=0 . V & V_{E}=-0.4 V \\ V_{C}=0 . V & V_{B}=0 . V & V_{E}=-0.6 V \\ V_{C}=0 . V & V_{B}=0 . V & V_{E}=-0.8 V \\ V_{C}=0 . V & V_{B}=0 . V & V_{E}=-1.0 V \\ V_{C}=0 . V & V_{B}=0 . V & V_{E}=-1.2 V \\ V_{C}=0 . V & V_{B}=0 . V & V_{E}=-1.4 V & \\ & & & \\ V_{C}=0 . V & V_{B}=-0.1 V & V_{E}=-1.4 V & \text {-see fig.(24)- } \\ V_{C}=0 . V & V_{B}=-0.2 V & V_{E}=-1.4 V & \\ V_{C}=0 . V & V_{B}=-0.3 V & V_{E}=-1.4 V \\ V_{C}=0 . V & V_{B}=-0.4 V & V_{E}=-1.4 V \\ \ldots & \ldots & \ldots\end{array}$


The convergence behaviour of algorithm 3 remain the same as for the quasi-1D case (previous section); for one example we present on fig.(24) corresponding results (curves) for the polarization $V_{C}=0 . \mathrm{V}, V_{B}=-0.1 \mathrm{~V} V_{E}=-1.4 \mathrm{~V}$. The other parameters are $\Delta V_{B}=-0.1 V, \bar{\lambda}_{i n i}=10, a=12, b=6$.

Let us now present some numerical results which are related to the physical behaviour of the heterojunction bipolar transistor. For these results, boundary layer model for the quasi-Fermi levels as in [32] is used and simulations have been carried out with different triangular meshes. The results presented here are for a mesh with 12445 triangles and 6417 vertices (see fig.(23)).

\subsubsection{Results when the "transistor effect" is not active}

For the bias values $V_{C}=0 . \mathrm{V}, V_{B}=-0.7 \mathrm{~V} V_{E}=-1.4 \mathrm{~V}$, the so-called transistor effect is not active. For this state we can see (on fig.(25) (at the top) that no current flow from Base to Emitter. On the previous figure we can see the current lines for the electronic current, for the hole current and for the (total) current. On figure (26) a view of the electron density distributions for the present polarization is given. Figure (27) gives a representation of the electron quasi-Fermi level $\Phi_{n}$ and figure (28) of the electrostatic potential.

\subsubsection{Results when the "transistor effect" is active}

Increasing the value of $V_{B}$, the transistor effect become to be active (from $\sim-0.5 \mathrm{~V}$ to $0 . V$ ). On figure (25) (at the bottom) we present the current lines for the bias point $V_{C}=0 . V, V_{B}=0 . V V_{E}=-1.4 \mathrm{~V}$. We can see now a current flowing from Base to Emitter. On figure (26) a view of the electron density distributions for the present polarization is given. Figure (27) gives a representation of the electron quasi-Fermi level $\phi_{n}$ and figure (28) of the electrostatic potential. 


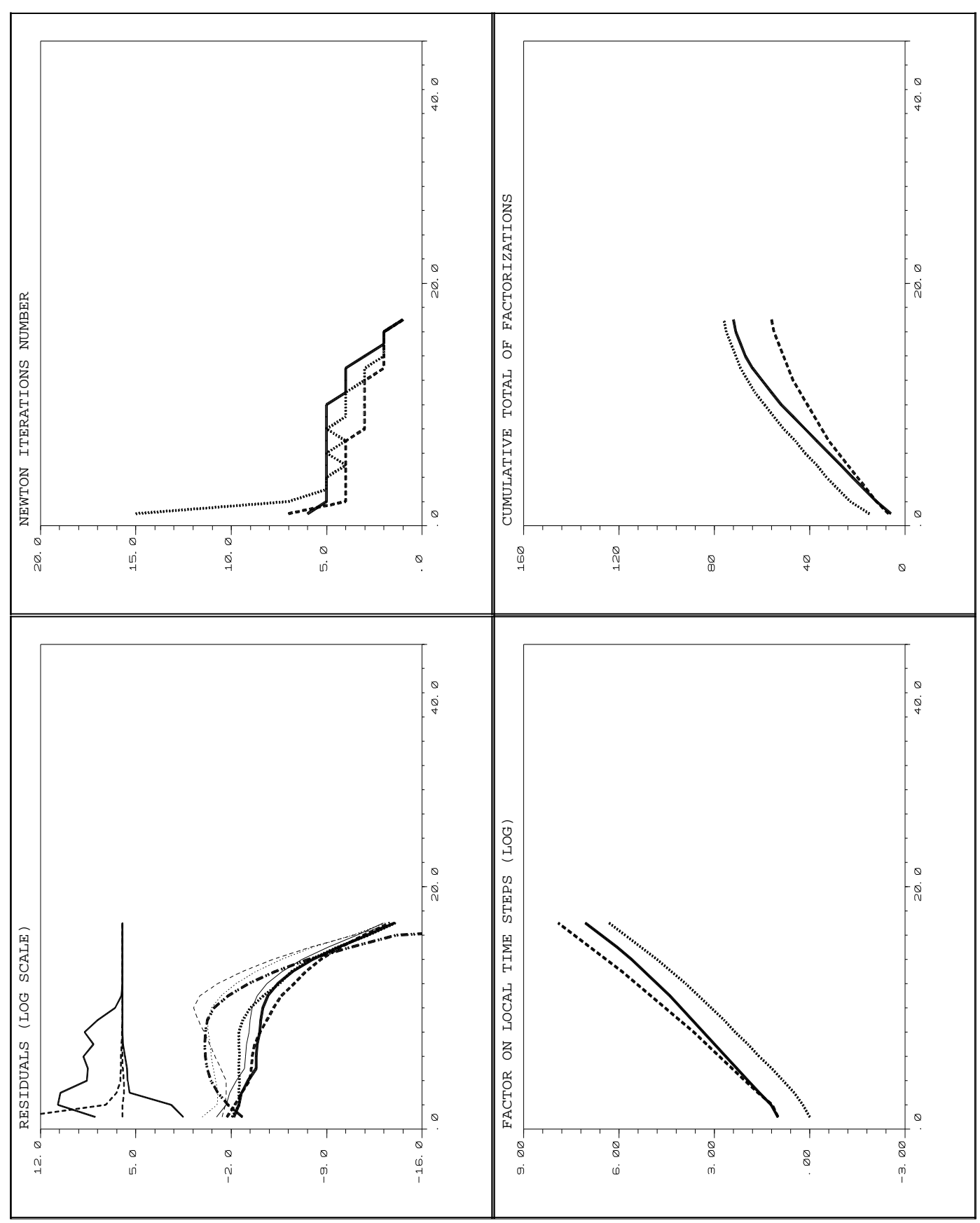

Figure 24: Algorithmic behaviour (Alg:3), $V_{C E}=1.4 \mathrm{~V}, \Delta V_{B}=-0.1 \mathrm{~V}, \bar{\lambda}_{i n}=10$, $a=12, b=6$

$\mathrm{RR} \quad \mathrm{n}^{\circ} 2546$ 

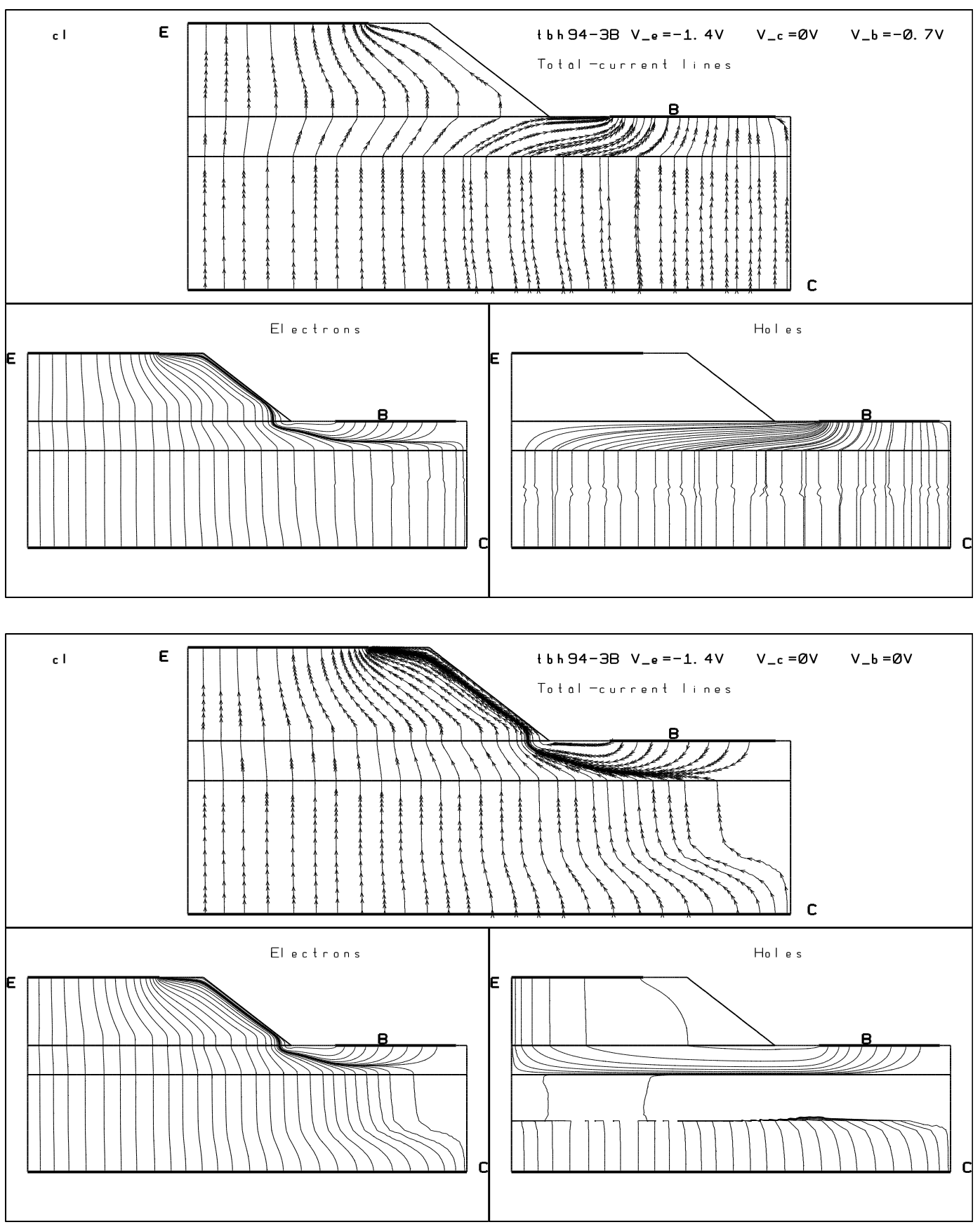

Figure 25: Numerical simulation for the H.B.T. Current lines. Graph at the top: without "transistor effect", at the bottom: "transistor effect" active. 

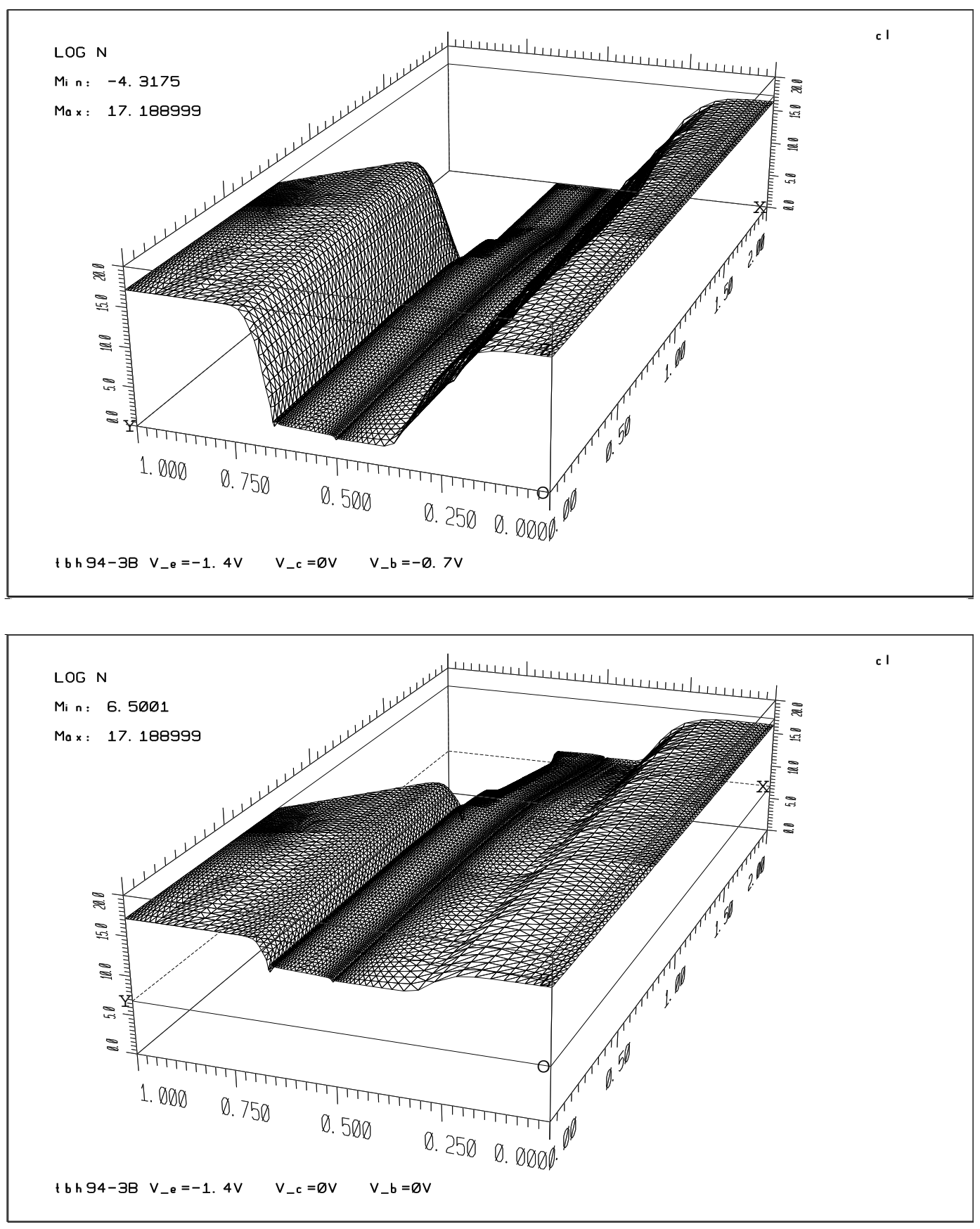

Figure 26: Numerical simulation for the H.B.T. Electron distributions. Graph at the top: without "transistor effect", at the bottom: "transistor effect" active.

$\mathrm{RR} \mathrm{n}^{\circ} 2546$ 

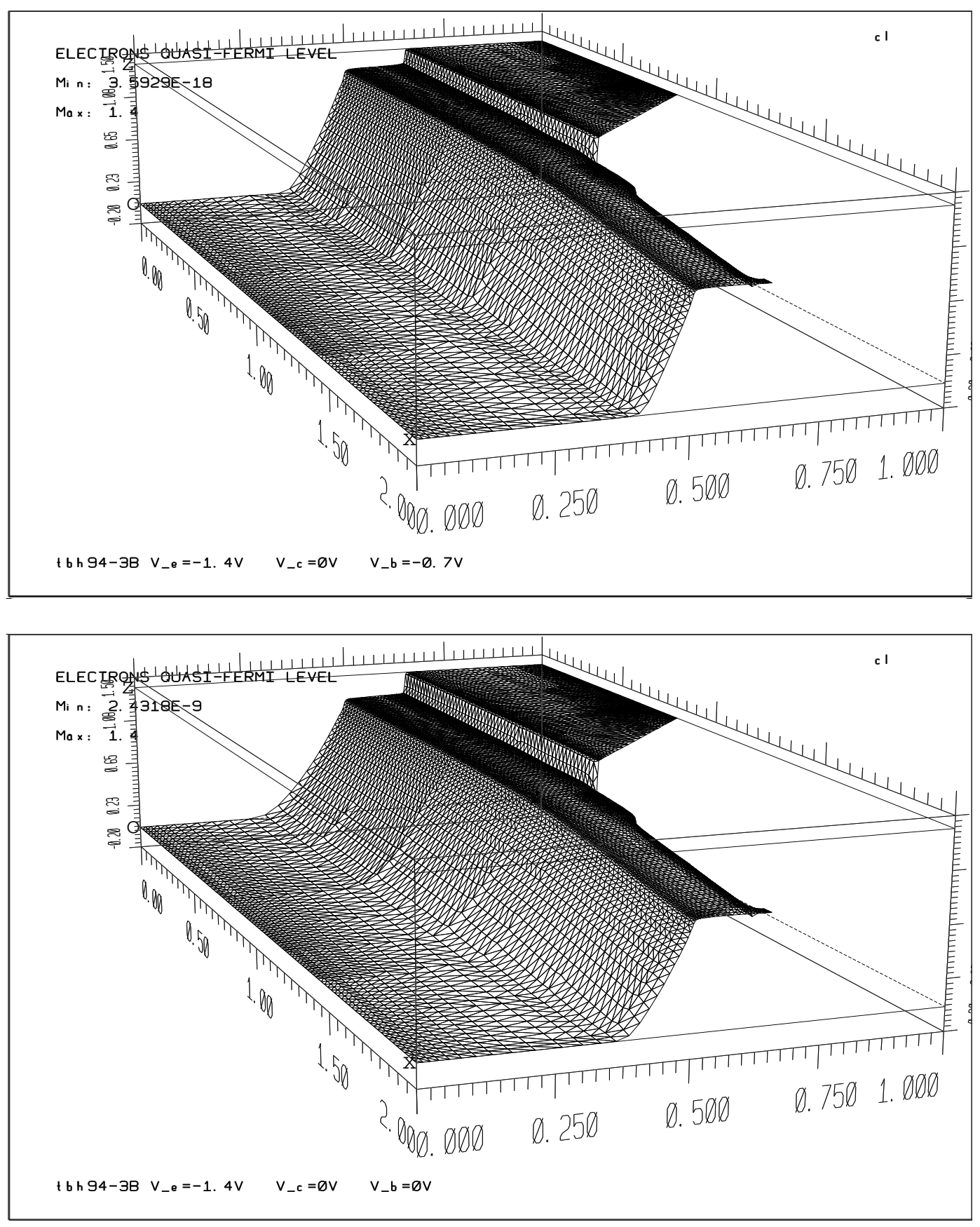

Figure 27: Numerical simulation for the H.B.T. Electron quasi-Fermi level $\phi_{n}$. Graph at the top: without "transistor effect", at the bottom: "transistor effect" active. 

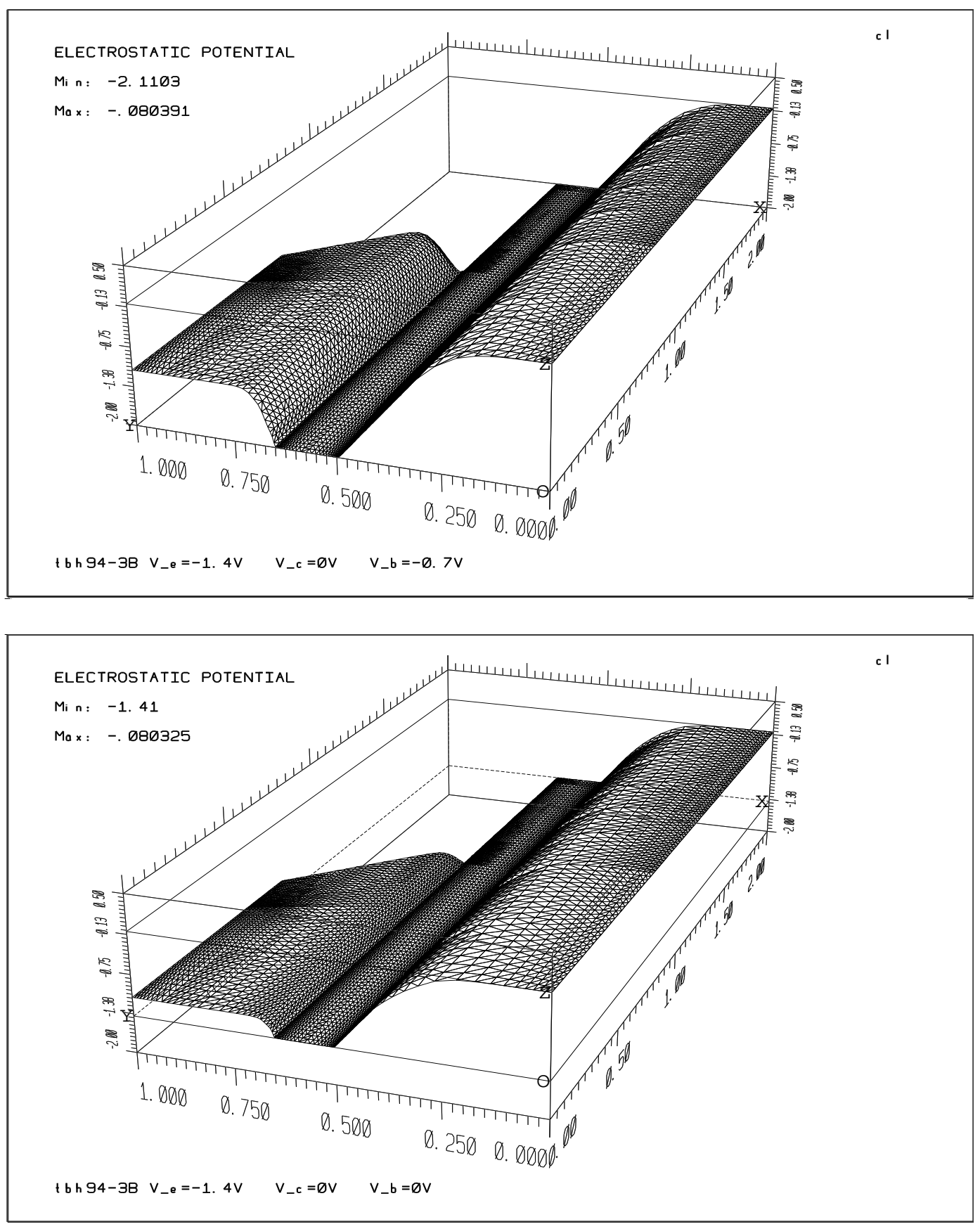

Figure 28: Numerical simulation for the H.B.T. Electrostatic potential $\phi$. Graph at the top: without "transistor effect", at the bottom: "transistor effect" active.

$\mathrm{RR} \mathrm{n}^{\circ} 2546$ 


\section{Conclusion}

We find that, the applications of the Arclength continuation type procedures (Algorithm 3 or Algorithm 2) give a robust and rigorous automatic adaptation of the time steps. For different increment voltage a good convergence, with reasonnable cost, is obtained. In the mentality of the time steps adaptation, these algorithms can be used for an automatic control of applied potentials, [51], and several implementation variants can be derived. For more rigorous strategies, in governing the steplengths $\Delta s_{i=1,2,3}$, one must combine the factors described in section 5 . From theoretical and numerical results, it appears that with these algorithms we can circumvent the unstabilities of the classical Newton-Raphson type algorithms which appear when the nonlinearity is strong or near limit or bifurcation points. Hence, they can be applied for more general semiconductor equations (which can encounter multiple solutions or turning points as in a Tyristor for example).

\section{Acknowledgement}

Authors thank A. Kaiss, P. LeTallec for fruitful discussions about continuation algorithms, and F. Hecht for system computer help. This work is supported by CNET (Bagneux) contract. 


\section{Contents}

1 Introduction $\quad 3$

2 Mixed variational formulation in $H(\operatorname{div}, \Omega) \times L^{2}(\Omega) \quad 7$

2.1 Mixed formulation . . . . . . . . . . . . . . 7

2.2 Dual mixed variational formulation in the continuous case . . . 8

2.3 Discretization of the mixed formulation . . . . . . . . . 9

2.4 Degrees of freedom of $V_{h}$ and mixed discretization . . . . . . . 10

2.5 Time discretization . . . . . . . . . . . . . . . 13

3 Basic concepts of the continuation method 18

3.1 Classical continuation method of Euler-Newton's type . . . . . . . 18

3.2 Algorithm 1 (Embedding Algorithm) ～. . . . . . . . . . . . 19

3.3 Parametrization and Arc-length continuation methods . . . . . . . 22

3.4 Choices of normalisations . . . . . . . . . . . . 27

3.5 Arclength continuation about regular and limit points . . . . . . 28

4 Detailed arclength continuation type algorithms $\quad 31$

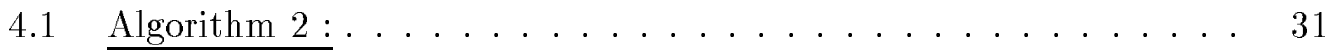

4.2 On the computation of the continuation velocities . . . . . . . 36

4.3 Solution procedure for the nonlinear sub-systems . . . . . . . . 40

4.4 On the solution of the linear systems . . . . . . . . . . . 46

4.5 Operational count for the algorithm $2 \ldots \ldots \ldots 46$

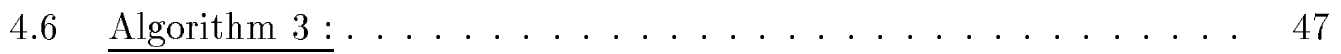

$5 \quad$ Steplength adaptation for the predictor step $\quad 53$

6 Numerical results $\quad \mathbf{5 7}$

6.1 Heterojunction diode . . . . . . . . . . . . . . 57

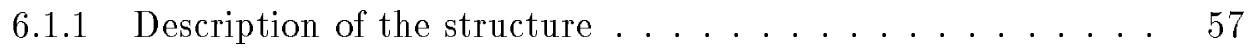

6.1 .2 Description of the solution figures . . . . . . . . 57

6.1.3 Numerical behaviour for algorithm 3 with respect to the voltage increment and the initial time steps . . . . . . . 61

6.1.4 Numerical behaviour for algorithm $2 \ldots \ldots 73$

6.2 Heterojunction Bipolar Transistor (H.B.T.) . . . . . . . . . . 78

6.2.1 Results when the "transistor effect" is not active . . . . . 80

6.2.2 Results when the "transistor effect" is active . . . . . . . 80 


\section{List of Figures}

1 Different possibilities for the curve $C(s) \ldots \ldots \ldots \ldots$

2 Raviart-Thomas mixed finite elements of low order. . . . . . . . . . 12

3 The classical Euler-Newton continuation. In this case the parameter $\lambda$ is suited as a parametrization for the curve. Instead of computing directly the solution for a given value of $\lambda$, we compute the solution for different values $\lambda_{i}$ distributed between the initial and the final loading. In this algorithm, the distance to the solution curve is not minimized.

$4 \quad$ Normal limit point. In this case, a classical Newton-Raphson's continuation type algorithms may generate a diverging sequence. Near this point, the Newton-Raphson's algorithm can't be quadratic. . . . . . .

5 Bifurcation points. a) Bifurcation at turning point. b) Bifurcation at limit point. In this situations, a classical Euler- Newton's type algorithms can't converge. The arclength continuation algorithm is necessary. . . . . . . . . . . . . . . . . . Strong nonlinearity. In this situation, the classical Euler-Newton's type algorithms can fail. The arclength continuation algorithm may be necessary. . . . . . . . . . . . . . . . . . .

$7 \quad$ Variations of the $\varepsilon_{0} c_{1}$ function with respect to the electrostatic potential $\phi$ and for different doping values. . . . . . . . . . . . .

Variations of the $c_{2}$ (Generation-Recombinaison term)function with respect to the electron quasi-Fermi level $\phi_{n}$ and for different values of the hole quasi-Fermi level $\phi_{p}$. Electrostatic potential value is kept to 0. 24

$9 \quad$ The values $v_{1}$, and $v_{2}$ of the applied potential correspond to two limit points. Besides, if $v_{1} \leq v \leq v_{2}$ multiple values may be obtained. In this case, the arclength continuation method is necessary. . . . . . .

10 Algorithm 2 : Arclength continuation type algorithm with constraint $N_{2}$. The suited path is not orthogonal to the velocity vector. . . . . .

11 Algorithm 2: Arclength continuation type algorithm with orthogonality constraint. The suited path is orthogonal to the velocity vector. .

12 Algorithm 3: Arclength continuation type algorithm. In this case, the velocity update is done within the Newton-Raphson's corrector steps. Then, the distance to the solution curve is minimized for each corrector steps. The number of corrector steps will be then less than the number in algorithm 2. . . . . . . . . . . . . . .

13 Velocity sign. In part $1, \dot{\lambda}_{1,2,3}$ are positive, but in part $2, \dot{\lambda}_{1,2,3}$ are negative. In limit point, $\dot{\lambda}_{1,2,3}=0 . \ldots \ldots \ldots \ldots$ 
14 Automatic steplength adatptation. The angle $\beta$ is a measure of the curvature. We have $\beta \in[0, \pi]$. When the nonlinearity is strong, the angle $\beta$ tends to zero, and when the nonlinearity is weak $\beta$ tends to $\pi$. The quantity $d$ is an approximation of the distance to the curve. The points 1, and 2 are the two first points in the corrector process.

15 Junction geometry for validation of our numerical results. The parts $\Gamma_{N 1, N 2}$ correspond to the two insulating boundaries (Neumann boundary conitions), and $\Gamma_{D 1, D 2}$ correspond to the two ohmic contacts (Dirichlet boundary conditions). . . . . . . . . . . . . .

16 Potential, quasi-Fermi levels, conduction and valence bands, carrier distributions. Graphs at the top: 0.25 V FORWARD BIAS, at the bottom: 1.V FORWARD BIAS . . . . . . . . . . .

17 Potential, quasi-Fermi levels, conduction and valence bands, carrier distributions. Graphs at the top: 0.25 V REVERSE BIAS, at

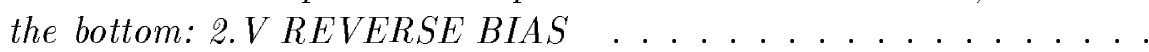

18 Algorithmic behaviour (Alg:3), ForwardBias : $0.5 \mathrm{~V}, \Delta V=0.5 \mathrm{~V}$, $\bar{\lambda}_{i n}=100, a=12, b=6 \ldots \ldots \ldots \ldots 6$

19 Algorithmic behaviour (Alg:3), ForwardBias : $1.5 \mathrm{~V}, \Delta \mathrm{V}=0.5 \mathrm{~V}$, $\bar{\lambda}_{i n}=100, a=12, b=6 \ldots \ldots \ldots \ldots \ldots$

20 Algorithmic behaviour (Alg:2), ForwardBias : $0.5 \mathrm{~V}, \Delta V=0.5 \mathrm{~V}$, $\bar{\lambda}_{i n}=100, a=12, b=6 \ldots \ldots \ldots \ldots$

21 Algorithmic behaviour (Alg:2), ForwardBias : $1.5 \mathrm{~V}, \Delta \mathrm{V}=0.5 \mathrm{~V}$, $\bar{\lambda}_{i n}=100, a=12, b=6 \ldots \ldots \ldots \ldots \ldots$

22 Description of the heterojunction bipolar transistor structure. . . . . 78

23 Triangular mesh on the H.B.T. . . . . . . . . . . . . .

24 Algorithmic behaviour (Alg:3), $V_{C E}=1.4 \mathrm{~V}, \Delta V_{B}=-0.1 \mathrm{~V}, \bar{\lambda}_{i n}=$ $10, a=12, b=6 \ldots \ldots \ldots \ldots \ldots$. . . . . . . . . 81

25 Numerical simulation for the H.B.T. Current lines. Graph at the top: without "transistor effect", at the bottom: "transistor effect"

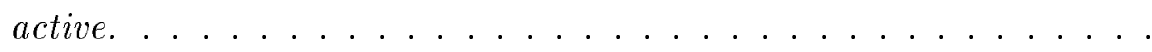

26 Numerical simulation for the H.B.T. Electron distributions. Graph at the top: without "transistor effect", at the bottom: "transistor

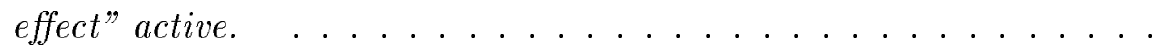

27 Numerical simulation for the H.B.T. Electron quasi-Fermi level $\phi_{n}$. Graph at the top: without "transistor effect", at the bottom: "transistor effect" active. . . . . . . . . . . . . . . 
28 Numerical simulation for the H.B.T. Electrostatic potential $\phi$. Graph at the top: without "transistor effect", at the bottom: "transistor

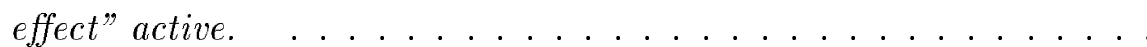

\section{List of Tables}

1 Summary of results (algorithm behaviour) Alg.3, Forward bias, $\Delta V=$

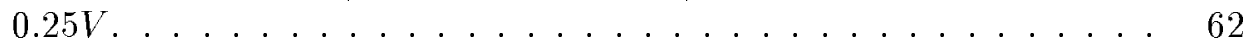

2 Summary of results (algorithm behaviour) Alg.3, Forward bias, $\Delta V=$

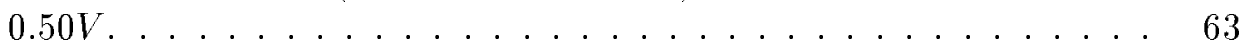

3 Summary of results (algorithm behaviour) Alg.3, Forward bias, $\Delta V=$

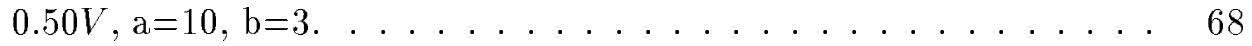

4 Summary of results (algorithm behaviour) Alg.3, Forward bias, $\Delta V=$

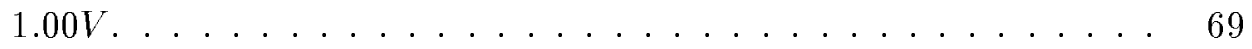

$5 \quad$ Summary of results (algorithm behaviour) Alg.3, Reverse bias, $\Delta V=$

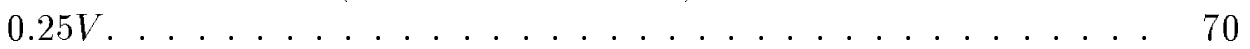

6 Summary of results (algorithm behaviour) Alg.3, Reverse bias, $\Delta V=$

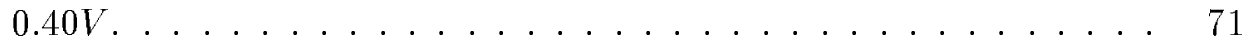

7 Carrier densities variations. Forward bias. . . . . . . . . . 73

8 Carrier densities variations. Reverse bias. . . . . . . . . . . . . 74

9 Summary of results (algorithm behaviour) Alg.2, Forward bias, $\Delta V=$

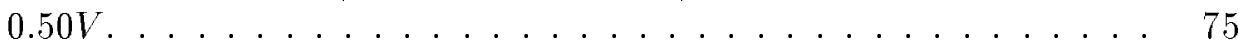




\section{Appendix}

Proof of theorem 3.1 Under suitable regularity assumptions, for $g_{1} \in H^{\frac{1}{2}}(\partial \Omega)$ there exits $\left(u^{0}, \lambda^{0}\right) \in W_{h} \times V_{0 h} \times \mathbb{R}$ such that the conditions 1) and 2) in (33) are verified.

Proof :

a) The condition 1) is verified thanks to the results derived in [30], [31], [29].

b) It remains to prove that the operator $H_{1}^{\prime}\left(u^{0}, \lambda^{0}\right)$ is onto from $W_{h} \times V_{0 h} \times \mathbb{R}$ to $\mathbb{R}^{N E+N N}$.

For all $\left(x_{1}, x_{2}\right) \in \mathbb{R}^{N E+N N}$,

$$
H_{1}^{\prime}\left(u^{0}, \lambda^{0}\right)\left(u^{1}, \lambda^{1}\right)=\left(x_{1}, x_{2}\right)
$$

is equivalent to the following equations

$$
\left\{\begin{array}{l}
\int_{\Omega} \alpha_{1}(x) D^{1} v d x+\int_{\Omega} \operatorname{div}(v) \phi^{1} d x=x_{1} \quad \forall v \in V_{0 h} \\
\int_{\Omega} \operatorname{div}\left(D^{1}\right) w d x-\int_{\Omega}\left[\frac{A_{1}}{\bar{\lambda}^{1}}+c_{1, \phi}^{\prime}\left(x, \phi^{0}\right)\right] \phi^{1} w d x=x_{2}-\int_{\Omega} \frac{A_{1} \phi^{0}}{\left(\bar{\lambda}^{1}\right)^{2}} w d x \forall w \in W_{h} .
\end{array}\right.
$$

To prove that the operator $H_{1}^{\prime}\left(u^{0}, \lambda^{0}\right)$ is onto, it is sufficient to prove that for all $\left(x_{1}, x_{2}\right) \in \mathbb{R}^{N E+N N}$ and for $\lambda^{1}$ given, we can find $\left(\phi^{1}, D^{1}\right) \in W_{h} \times V_{0 h}$ which verify the problem (125). Setting

$$
\bar{x}_{2}=x_{2}-\int_{\Omega} \frac{A_{1} \phi^{0}}{\left(\bar{\lambda}^{1}\right)^{2}} w d x
$$

and consider the operator $P_{\lambda^{1}}: W_{h} \times V_{0 h} \rightarrow \mathbb{R}^{N E+N N}$ defined by

$$
P_{\lambda^{1}}\left(\phi^{1}, D^{1}\right)=\left(x_{1}, \bar{x}_{2}\right) .
$$

We prove that $P_{\lambda^{1}}$ is injective. Since we are in finite dimension and the latter operator is linear and continuous we conclude that this one is onto. We have

$$
P_{\lambda^{1}}\left(\phi^{1}, D^{1}\right)=0
$$

implies

$$
\left\{\begin{array}{l}
\int_{\Omega} \alpha_{1}(x) D^{1} v d x+\int_{\Omega} \operatorname{div}(v) \phi^{1} d x=0 \quad \forall v \in V_{0 h} \\
\int_{\Omega} \operatorname{div}\left(D^{1}\right) w d x-\int_{\Omega}\left[\frac{A_{1}}{\bar{\lambda}^{1}}+c_{1, \phi}^{\prime}\left(x, \phi^{0}\right)\right] \phi^{1} w d x=0 \forall w \in W_{h} .
\end{array}\right.
$$

$\mathrm{RR} \mathrm{n}^{\circ} 2546$ 
We define the bilinear form a(.,.), b(.,.) and $c_{0}(.,$.$) as follows :$

$$
\begin{gathered}
a\left(D^{1}, v\right)=\int_{\Omega} \alpha_{1}(x) D^{1} v d x, \forall D^{1}, v \in V_{0 h}, \\
b\left(v, \phi^{1}\right)=\int_{\Omega} \operatorname{div}(v) \phi^{1} d x, \quad \forall v \in V_{0 h}, \forall \phi^{1} \in W_{h}, \\
c_{0}\left(\phi^{1}, w\right)=\int_{\Omega}\left[\frac{A_{1}}{\bar{\lambda}^{1}}+c_{1, \phi}^{\prime}\right] \phi^{1} w d x, \quad \forall\left(\phi^{1}, w\right) \in W_{h}^{2} .
\end{gathered}
$$

We associate for each bilinear forms defined above the following linear and continuous opertators :

$$
A: V_{0 h} \rightarrow V_{0 h^{\prime}}{ }^{\prime}, \quad B: V_{0 h} \rightarrow W_{h}{ }^{\prime}, B^{t}: W_{h} \rightarrow V_{0 h}^{\prime}, \quad C: W_{h} \rightarrow W_{h}{ }^{\prime}
$$

defined by

$$
\begin{gathered}
\left\langle A D^{1}, v\right\rangle=a\left(D^{1}, v\right) \forall\left(D^{1}, v\right) \in V_{h 0}^{2}, \\
\langle B v, w\rangle=b(v, w) \forall(v, w) \in V_{0 h} \times W_{h}, \\
\left\langle B^{t} w, v\right\rangle=<w, B v>\quad \forall(v, w) \in V_{0 h} \times W_{h}, \\
\left\langle C \phi^{1}, w\right\rangle=c_{0}\left(\phi^{1}, w\right) \quad \forall\left(\phi^{1}, w\right) \in W_{h}^{2} .
\end{gathered}
$$

With the above operators, the problem (126) can be written as follows

$$
\left\{\begin{array}{l}
A D^{1}+B^{t} \phi^{1}=0, \\
B D^{1}+C \phi^{1}=0,
\end{array}\right.
$$

Under hypothesis (H1), and since the operator $A_{1}$ is bounded, the operator $\mathrm{C}$ is coercive :

$$
\langle C w, w\rangle \geq \beta_{1}\|w\|_{0}^{2} \forall w \in W_{h},
$$


where $\beta_{1}=\frac{A_{1}^{0}}{L}+\beta_{0}$. Thanks to Lax-Milgram theorem [45], C is invertible on $W_{h}$. Besides, the application of the Open Map [46], [47], implies that $C^{-1}$ is continuous on $W_{h}$. The problem (127) is now equivalent to the following

$$
\left\{\begin{array}{l}
{\left[A+B^{t} C^{-1} B\right] D^{1}=0} \\
\phi^{1}=C^{-1} B D^{1}
\end{array}\right.
$$

It is sufficient now to prove that the operator $A+B^{t} C^{-1} B$ is invertible. We have

$$
\langle A v, v\rangle+\left\langle B^{t} C^{-1} B v, v\right\rangle=a(v, v)+\left\langle C^{-1} B v, B v\right\rangle \forall v \in V_{0 h} .
$$

We recall here that the norm in $V$ is given by

$$
\|v\|_{V}^{2}=\|v\|_{0}^{2}+\|B(v)\|_{0}^{2} .
$$

Since $\alpha_{1}$ is bounded, the bilinear form a(.,.) is coercive in $W_{h}$ :

$$
a(v, v) \geq \alpha_{1}^{0}\|v\|_{0}^{2} \forall v \in V_{0 h} .
$$

It remains to prove that $C^{-1}$ is coercive.

Lemma .1 The operator $C^{-1}$ is coercive in $W^{\prime}=W$ : there exists $\beta_{2}>0$ such that

$$
\left\langle C^{-1} q, q\right\rangle \geq \beta_{2}\|q\|_{W^{\prime}}^{2} \forall q \in W^{\prime} .
$$

Thanks to the inequalities (130) and (131), we have

$$
\begin{aligned}
\left\langle\left(A+B^{t} C^{-1} B\right) v, v\right\rangle & \geq \alpha_{1}^{0}\|v\|_{0}^{2}+\beta_{2}\|B v\|_{0}^{2} \forall v \in V_{0 h} \\
& \geq \operatorname{Min}\left(\alpha_{1}^{0}, \beta_{2}\right)\left(\|v\|_{0}^{2}+\|B v\|_{0}\right) \quad \forall v \in V_{0 h} \\
& \geq \operatorname{Min}\left(\alpha_{1}^{0}, \beta_{2}\right)\|v\|_{V}^{2} \quad \forall v \in V_{0 h} .
\end{aligned}
$$

Thanks to Lax-Milgram theorem [47], the operator $A+B^{t} C^{-1} B$ is invertible. Hence, 
the operator $P_{\lambda^{1}}$ is injective. We have

$$
W_{h} \times V_{0 h} / \operatorname{Ker}\left(P_{\lambda^{1}}\right) \simeq \operatorname{Im}\left(P_{\lambda^{1}}\right) \subset \mathbb{R}^{N E+N N} .
$$

This implies that $P_{\lambda^{1}}$ is onto from $W_{h} \times V_{0 h}$ to $\mathbb{R}^{N E+N N}$.

\section{Proof of lemma .1 :}

We have $W^{\prime} \equiv W$. Let $q \in W^{\prime}$, since the operator $\mathrm{C}$ is invertible from $\mathrm{W}$ to $W^{\prime}$, there exists $p \in W$ such that $\mathrm{Cp}=\mathrm{q}$.

$\mathrm{C}$ is coercive in $\mathrm{W}$ then

$$
\left\langle C^{-1} q, q\right\rangle=\langle p, C p\rangle \geq \beta_{1}\|p\|_{0}^{2}
$$

We have

$$
\left\langle C p, q_{1}\right\rangle=\int_{\Omega}\left[\frac{A_{1}}{\bar{\lambda}^{1}}+c_{1, \phi}^{\prime}(x, \phi)\right] p q_{1} d x
$$

From hypothesis (H2), we deduce

$$
\|C p\|_{W^{\prime}} \leq M\|p\|_{W} \quad \forall p \in W .
$$

Hence,

$$
\|p\|_{W}^{2} \geq \frac{1}{M^{2}}\|C p\|_{W^{\prime}}^{2}
$$

and then

$$
\|p\|_{W}^{2} \geq \frac{1}{M^{2}}\|q\|_{W^{\prime}}^{2}
$$

Thanks to the inequalities (134) and (138), we have

$$
\left\langle C^{-1} q, q\right\rangle \geq \frac{\beta_{1}}{M^{2}}\|q\|_{W^{\prime}}^{2} .
$$

We conclude that $C^{-1}$ is coercive.

Proposition .1 Under suitable assumptions and for $g_{2} \in H^{\frac{1}{2}}(\partial \Omega)$ and for $h$ sufficiently small, the operator $H_{2, u_{2}}^{0}$ given in (56)is nonsingular. 


\section{Proof of propostion .1 :}

The opertor $H_{2, u_{2}}^{0}$ is linear, since we are in finite dimensional case, it is sufficient to prove that this operator is injective. Setting

$$
u_{2}^{0}=\left(\phi_{n}^{0}, j_{n}^{0}\right) \quad u_{2}=\left(\phi_{n}, j_{n}\right)
$$

We have

$$
H_{2, u_{2}}^{0} \cdot\left(u_{2}\right)=0
$$

is equivalent to the following equations

$$
\left\{\begin{array}{l}
\left(\alpha_{2}\left(x, \phi_{n}^{0}\right) j_{n}, v_{2}\right)+\left(\operatorname{div}\left(v_{2}\right), \phi_{n}\right)+\left(\Gamma(x) \phi_{n}, v_{2}\right)=0 \quad \forall v_{2} \in V_{0 h}, \\
\left(\operatorname{div}\left(j_{n}\right), w_{2}\right)-\left(\gamma(x) \phi_{n}, w_{2}\right)=0 \quad \forall w_{2} \in W_{h},
\end{array}\right.
$$

where

$$
\begin{aligned}
& \alpha_{2}\left(x, \phi_{n}^{0}\right)=\alpha_{2}\left(x, \phi^{1}, \phi_{n}^{0}\right), \\
& \Gamma(x)=\alpha_{2, \phi_{n}}^{\prime}\left(x, \phi_{n}^{0}\right) j_{n}^{0} \\
& \gamma(x)=\frac{A_{2}(x)}{\Delta t}+c_{2, \phi_{n}}^{\prime}\left(x, \phi^{1}, \phi^{0}, \phi_{p}^{0}\right) .
\end{aligned}
$$

In order to prove the proposition .1, we shall use the following technical result (duality lemma) derived in [49], [50], [30].

Lemma .2 Let $2 \leq \theta<\infty$. Let $j_{n} \in V, q \in L^{2}(\Omega)^{2}$, and $r \in L^{2}(\Omega)$. If $\phi_{n} \in W_{h}$ satisfies

$$
\left\{\begin{array}{l}
\left(\alpha_{2}\left(x, \phi_{n}^{0}\right) j_{n}, v_{2}\right)+\left(\operatorname{div}\left(v_{2}\right), \phi_{n}\right)+\left(\Gamma(x) \phi_{n}, v_{2}\right)=\left(q, v_{2}\right) \forall v_{2} \in V_{0 h}, \\
\left(\operatorname{div}\left(j_{n}\right), w_{2}\right)-\left(\gamma(x) \phi_{n}, w_{2}\right)=\left(r, w_{2}\right) \forall w_{2} \in W_{h}
\end{array}\right.
$$

then, there exists a constant $C=C\left(\theta, \alpha_{2}, \Gamma, \gamma, \Omega\right)$ such that

$$
\left\|\phi_{n}\right\|_{0, \theta} \leq C\left[h^{\frac{2}{\theta}}\left\|j_{n}\right\|_{0}+h^{1+\left(\frac{2}{\theta}\right)\left(1-\sigma_{0 k}\right)}\left\|\operatorname{div}\left(j_{n}\right)\right\|_{0}+\|q\|_{0}+\|r\|_{0}\right] .
$$

for h sufficiently small. 
Here, $\sigma_{i j}$ denotes the Kronecker symbol.

If we take $w_{2}=\operatorname{div}\left(j_{n}\right)$ in the second equation of $(140)$, since $\gamma$ is bounded (hypothesis (H3)), we obtain

$$
\left\|\operatorname{div}\left(j_{n}\right)\right\|_{0} \leq Q\left\|\phi_{n}\right\|_{0}
$$

The application of the following duality lemma with $\theta=2, q=r=0, k=0$ implies

$$
\begin{aligned}
\left\|\phi_{n}\right\|_{0} & \leq Q\left\{h\left\|j_{n}\right\|_{0}+h\left\|\operatorname{div}\left(j_{n}\right)\right\|_{0}\right\}, \\
& \leq Q\left\{h\left\|j_{n}\right\|_{0}+h\left\|\phi_{n}\right\|_{0}\right\} .
\end{aligned}
$$

For h sufficiently small, we obtain

$$
\left\|\phi_{n}\right\|_{0} \leq Q h\left\|j_{n}\right\|_{0}
$$

Now we take $v_{2}=j_{n}$ in the first equation of (140). It follows from assumption (H3) that $\alpha_{2}, \alpha_{2, \phi_{n}}^{\prime}$, and $j_{n}^{0}$ are bounded. Hence, we have

$$
\left\|j_{n}\right\|_{0}^{2} \leq Q\left\{\left\|\operatorname{div}\left(j_{n}\right)\right\|_{0}\left\|\phi_{n}\right\|_{0}+\left\|\phi_{n}\right\|_{0}\left\|j_{n}\right\|_{0}\right\} .
$$

Thanks to the inequalities (144) and (146), we deduce

$$
\left\|j_{n}\right\|_{0} \leq Q\left\|\phi_{n}\right\|_{0} \leq Q h\left\|j_{n}\right\|_{0} .
$$

Using the above, for $\mathrm{h}$ sufficiently small, $j_{n}=0$ and consequently $\phi_{n}=0$. 


\section{References}

[1] H. POINCARE (1881-1886), Sur les courbes définies par une équation différentielle. I-IV. in: Oeuvres I. Gauthier-Villars, Paris,

[2] F. KLEIN (1882-1883), Neue Breitrage zur Riemannschen functionentheorie. Math. Ann. 21.

[3] S. BERNSTEIN (1910), Sur la généralisation du problème de Dirichlet. Mat. Ann., Vol. 69, pp. 82-136.

[4] J. LERAY, J. SCHAUDER (1934), Topologie et équations fonctionnelles. Ann. Sci. Ecole Norm. Sup. Vol. 51, pp. 45-78.

[5] E. LAHAYE (1934), Une méthode de résolution d'une categorie d'équations transcendantes. C. R. Acad. Sci. Paris, Vol. 198, 1840-1842.

[6] M.S. MOCK, Analysis of mathematical models of semiconductor devices. Dublin, Boole Press (1983).

[7] P.A. MARKOWICH, The stationary semiconductor device equations. Wien, New-York, Springer Verlag (1986).

[8] S. M. SZE (1981), Physics of semiconductor devices. New-York, John Wiley and Sons, 2nd edition, A Wiley interscience publication.

[9] W. VANN ROOSBROECK (1950), Theory of flow of electrons and holes in germanium and other semiconductors. Bell Sys. Tech. J., Vol. 29, 1950, pp. 560-607

[10] S. SELBERHERR, Analysis and simulation of semiconductor devices, Springer Verlag, Wien (1984).

[11] W. HANSCH, M. MIURA-MATTAUSCH (1986), The hot-electron problem in small semiconductor devices. J. Appl. Phys. 80, pp. 650-565.

[12] J.E. VIALLET, Simulation temporelle de dispositifs à hétérojonctions: mise en équation et méthode numériques, Thèse de Doctorat ès Sciences Physiques, Paris 6, (1986).

[13] M. FILOCHE, E. CAQUOT, J.F. PALMIER, Ether: Logiciel de simulation unidimensionnelle du comportement électrique des dispositifs semiconducteurs à hétérojonctions, Publication CNET, (1988).

[14] P.A. RAVIART and J.M. THOMAS, A mixed finite element method for and order elliptic problems. In Mathematical Aspects of Finite Element Methods (I.Galligani and E.Magenes Ed), Lecture notes 606, Springer Verlag, pp. 292-315 (1977).

[15] V. GIRAULT, P.A. RAVIART, Finite element methods for Naviers-Stokes equations. Theory and Algorithms, Springer Verlag, Berlin, Heidelbeg, New-York, Tokyo, (1986).

[16] S.J. POLACK, A finite element method with current conservation. SISDEP, Proceeding Bologne (Italy), Sept., (1988).

$\operatorname{RR~} n^{\circ} 2546$ 
[17] O. PIRONNEAU, Finite element methods for fluids, John Wiley and Sons (1989).

[18] P. LE TALLEC, Existence and Uniqueness Results for nonlinear mixed problems : Application to uncompressible Finite Elasticity. Numer. Math., Vol 38, 1982, pp. 365-382.

[19] J.W. JEROME, Consistency of semiconductor modeling: An existence stability analysis for the stationary Van Roosbroeck system. SIAM J. Appl. Math. V. 45, $n^{0} 4$ (1985).

[20] K. SOUISSI (1989), Simulation numérique des dispositifs électroniques à hétérojonctions. Thèse de Doctorat de l'Université Paris VI.

[21] F. BREZZI, A.C. CAPELO and L. GASTALDI, A singular perturbation analysis for the reverse-biased diodes. SIAM J. Math. Anal., V. 19 (5) (1989).

[22] F. ALABAU (1987), Analyse asymptotique et simulation numérique des équations de base des semiconducteurs. Thèse de Doctorat de l'Université Paris VI.

[23] F. BREZZI, M. FORTIN, Mixed and Hybrid Finite Element methods, Springer Verlag (1991).

[24] F. BREZZI, J. DOUGLAS, Jr. L. D. MARINI, Two families of mixed finite elements for 2nd order elliptic problems. Numer. Mth. Vpl., V. 47, pp. 217-235 (1985).

[25] J. DOUGLAS, Jr., J.E. ROBERT, Mixed finite element methods for second order elliptic problems. Math. Applic., V. 1, pp. 91-103 (1982).

[26] J. STOER (1983), Solution of large linear systems of equations by conjugate gradient type methods. In Mathematical programming : The state of the art. A. Bachem, M. Grotschel, B. Korte editors. Spring Verlag, Berlin, Heidelberg, New-York, pp. 540-565.

[27] M. ORTHEGA, W. C. RHEINBOLDT (1970), Iterative solution of nonlinear equations in several variables. New York: Academic Press.

[28] A. EL BOUKILI, A. MARROCCO, Mixed finite element approach and nonlinear implicit schemes for Drift-Diffusion equation solution of $2 D$ heterojunction semiconductor devices. Accepted in : International Journal for Computation and Mathematics in Electrical and Electronic Engineering, December 1994.

[29] A. EL BOUKILI (1995), Analyse mathématique et simulation numérique bidimensionnelle des dispositifs semiconducteurs à hétérojonctions par l'approche éléments finis mixtes. Thèse de Doctorat de l'Université Paris VI.

[30] A. EL BOUKILI, Analyse mathématique d'un problème mixte non linéaire et non standard issu des équations de semiconducteurs à hétérojonctions en statistique de FermiDirac. Rapport de recherche, INRIA, a paraître.

[31] A. EL BOUKILI, An application of Hadamard Theorem to a nonlinear mixed problem arising from semiconductor equations. To be published. 
[32] F. HECHT, A. MARROCCO, Mixed finite element simulation of heterojunction structures including a boundary layer model for the quasi-fermi levels. Proceedings of NASECODE X Conference, DUBLIN, June 21, (1994).

[33] H. B. KELLER (1977), Numerical solution of bifuraction and nonlinear eigenvalue problem. In: Application of bifurcation theory, Academic Press New York, pp. 359-384.

[34] H. B. KELLER (1982), Continuation methods in computational fluid dynamics. Numer. Phys. Asp. Aero. low. T. Cedeci editor. Spring Verlag, Berlin, Heidelberg, New York, pp. 3-13.

[35] M. A. CRISFIEL (1980), Incremental/Iterative solution procedures for nonlinear structural analysis. In: Numerical methods for nonlinear problems. Vol. 1. C. Taylor and E. Hinton and D. R. J. Owen editors. Pineridge Press, Swansea, UK.

[36] M. A. CRISFIEL (1983), An arc-length method incuding line searches and accelerations. Intermat. J. Numer. Methods Engrg. Vol. 19, 1269-1289.

[37] M. A. CRISFIEL (1991), Nonlinear finite element analysis of solids and strucures. Vol. 1, John Wiley and Sons, Chichester, England, ISBN 047-929565.

[38] E. RIKS (1992), Mechanical stability and buckling summer school for numerical analysis. At Le Bréau. Lectures notes part II.

[39] E. RIKS (1987), Bordered equations in solution technique. NLR MP 8705570, National Aerospace Lab. NRL, THe Netherland.

[40] E. RIKS (1984), Some computational aspects of the stability analysis of nonlinear structures. Comp. Meth. In Appl. Mehc. In Engng. Vol. 47, pp. 219-259.

[41] F. FICKEN (1951), The continuation method for functional equations. Comm. Pure Appl. Math. Vol. 4, pp.435-456.

[42] E. WASSERSTROM (1973), Numerical solution by the continuation method. SIAM Rev. Vol. 15, pp. 89-119.

[43] H. J. WACKER (1978), Continuation methods. Academic Press, New York, London.

[44] E. RIKS, An incremental approach to the solution of snapping and buckling problems. Internat. J. Solids Structures 15, 1979, pp. 529-551.

[45] S. B. MELVYN, An introduction to nonlinear Analysis. W. A. Benjamin INC (1968).

[46] H. BREZIS, Analyse fonctionnelle, Théorie et Application. Masson, Paris 1988.

[47] S. B. MELVYN, Nonlineartity and Functional Analysis. Lectures on Nonlinear Problems in Mathematical Analysis (1982).

[48] J. W. MILNOR (1969), Topology from the differentiable viewpoint. Univ. Press of Virginia, Charlottesville, Va. 
[49] J. DOUGLAS, Jr., J.E. ROBERT, Global estimates for mixed methods for second order elliptic equations. Math. Comp., V. 44, pp. 39-52 (1985).

[50] F. A. MILNER, Mixed finite elements for quasilinear second-order elliptic problems. Math. Comput, Vol. 44, $n^{\circ}$ 170, April 1985, pp. 303-320.

[51] A. EL BOUKILI, A. MARROCCO, Automatic adaptation of applied potential using an arclength continuation algorithm for semiconductor devices. To be published.

[52] E. POLAK, G. RIBIERE (1969), Note sur la convergence de méthodes de directions conjuguées. Rev. Française, Informt., Recherche opérationelle, Vol. 3, pp. 35-43.

[53] D. P. BERTSEKAS (1984), Constrained optimization and Lagrange multiplier methods. Academic Press, New York, London.

[54] M. J. D. POWELL (1977), Restart procedures for the conjugate gradient method. Math. Programming, Vol. 12, 241-254.

[55] R. GLOWINSKI, H. B. KELLER, L. REINHART (1985), Continuation-conjugate gradient methods for the least squares solution of nonlinear boundary value problems. SIAM J. SCi. Statist. Comput. Vol. 6, pp. 793-832.

[56] H. B. KELLER (1982), The bordering algorithm and path following near singular points of higher nullity. SIAM J. SCi. Statics. Comput. Vol. 4, pp. 573-582.

[57] P. LE TALLEC, Numerical methods for nonlinear three-dimensional elasticity. Handbook of Numerical Analysis, Vol. III, 1994, pp. 465-622.

[58] A. KAISS, La modélisation du comportement viscoélastique de l'œil et du contact ailtrépan. Thèse de l'Université Paris-IX Dauphine, 1994.

[59] A. EL BOUKILI, A new modified Euler continuation type algorithm for semiconductor equations with mixed finite element approach. To be published.

[60] J. MOLENAAR, P.W. HEMCKER, A multigrid approach for the solution of $2 D$ semiconductor equations. IMPACT of Computing in Science and Engineering 2,219-243 (1990). 


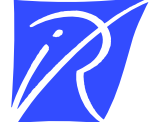

Unité de recherche INRIA Lorraine, Technopôle de Nancy-Brabois, Campus scientifique, 615 rue du Jardin Botanique, BP 101, 54600 VILLERS LES NANCY

Unité de recherche INRIA Rennes, Irisa, Campus universitaire de Beaulieu, 35042 RENNES Cedex Unité de recherche INRIA Rhône-Alpes, 46 avenue Félix Viallet, 38031 GRENOBLE Cedex 1

Unité de recherche INRIA Rocquencourt, Domaine de Voluceau, Rocquencourt, BP 105, 78153 LE CHESNAY Cedex

Unité de recherche INRIA Sophia-Antipolis, 2004 route des Lucioles, BP 93, 06902 SOPHIA-ANTIPOLIS Cedex

Éditeur

INRIA, Domaine de Voluceau, Rocquencourt, BP 105, 78153 LE CHESNAY Cedex (France)

ISSN 0249-6399 